\title{
RADPOS System as a Dose and Position Quality Assurance Tool for 4D Radiotherapy with CyberKnife
}

\author{
by
}

Raanan Marants

A thesis submitted to the Faculty of Graduate and Postdoctoral Affairs in partial fulfillment of the requirements for the degree of

\author{
Master of Science \\ in \\ Physics (Medical Physics stream) \\ Carleton University \\ Ottawa, Ontario \\ (C) 2015, Raanan Marants
}




\begin{abstract}
The CyberKnife system consists of a compact linear accelerator mounted on a mobile robotic arm. It uses the Synchrony Motion Tracking System to compensate for respiratory motion. This complex radiotherapy system needs independent performance verification to assure safe treatments. In this work, we use the RADPOS 4D dosimetry system to verify CyberKnife's motion tracking and delivered dose. RADPOS motion measurements are compared with Synchrony log files containing internal metal fiducial positions determined using orthogonal $\mathrm{kV}$ x-ray imaging, and external LED marker positions determined using a stereoscopic camera system. RADPOS dose measurements are compared with radiochromic film measurements and treatment planning system calculations. The standard deviation of the differences between RADPOS and LED position measurements, as well as between RADPOS and fiducial position measurements, were less than $1 \mathrm{~mm}$ along all directions of motion. Percent comparisons between the treatment planning system, RADPOS, and film dose values were mostly within 2 standard deviations of experimental uncertainties. Our work demonstrates that the RADPOS system is a useful tool for the independent quality assurance of CyberKnife treatments.
\end{abstract}




\section{Acknowledgements}

I would like to begin by thanking my supervisor Joanna Cygler and co-supervisor Eric Vandervoort for their continual guidance and support over the course of my MSc. In addition to providing me with a seemingly endless supply of knowledge, they helped develop my technical skills, critical thinking and professionalism as a scientist, for which I am very grateful. I look forward to applying all that I have learned from them as I continue on in academia.

Thanks to mechanical technologists Bernie Lavigne and Ron Romain and radiation therapist Steve Andrusyk for their help with various aspects of our experiments.

I would like to acknowledge and thank my colleague Miro Vujicic, with whom I had many conversations regarding my research work. Many successful aspects of my thesis stemmed from ideas conceived during our talks.

Thank you to Amanda Cherpak for setting an excellent foundation for me to use as I began my thesis work. Her detailed and extensive notes and manuals were extremely helpful when it came to familiarizing myself with the technology and system I would be working with.

I would like to thank my nominal co-supervisor Paul Johns for his help and advice throughout my MSc.

Finally, thank you to my family and friends for their unwavering support over these past couple of years. Five hour trips between Toronto and Ottawa are no longer an issue for them. 


\section{Statement of Originality}

I performed the data analysis for the various experiments. In addition, I wrote the MATLAB programs for: importing the data from CyberKnife log files and RADPOS output files, implementing the coordinate alignment algorithm, and analyzing certain aspects of the positional and dosimetric results. The film analysis scripts, involving film calibration and dose analysis, were written by Eric Vandervoort.

I was responsible for preparing the apparatus for the various experiments that were performed. This included measuring, cutting, and pre-scanning the film, and setting up the hardware and software for RADPOS and the Quasar phantoms. Quasar modifications were done by Bernie Lavigne and Ron Romain. Pre-experiment CT scans were done by Steve Andrusyk, with phantom placement and setup done by me, Eric Vandervoort, and Joanna Cygler. Experiments were performed by me, Eric Vandervoort (operated the CyberKnife) and Joanna Cygler (oversaw the experiment).

I have presented this work as an oral presentation at the Ottawa Medical Physics Institute (OMPI) seminar series, the Ottawa Carleton Institute for Physics (OCIP) seminar series, and the 2015 World Congress on Medical Physics and Biomedical Engineering (WC 2015). In addition, I have presented this work as a poster at the 2014 Canadian Organization of Medical Physicists Annual Scientific Meeting (COMP 2014) and at the 2015 American Association of Medical Physicists Annual Meeting and Exhibition (AAPM 2015). 


\begin{tabular}{|l|l|}
\hline COMP 2014 & $\begin{array}{l}\text { Marants, R., Vandervoort, E., Cygler, J. E. Dose and Position Quality } \\
\text { Assurance using the RADPOS System for 4D Radiotherapy with } \\
\text { CyberKnife. Med Phys 41, 2014 }\end{array}$ \\
\hline OMPI & $\begin{array}{l}\text { Marants, R., Vandervoort, E., Cygler, J. E. Dose and Position Quality } \\
\text { Assurance using the RADPOS System for 4D Radiotherapy with } \\
\text { CyberKnife. Ottawa, Canada, 2015 }\end{array}$ \\
\hline OCIP & $\begin{array}{l}\text { Marants, R., Vandervoort, E., Cygler, J. E. Application of RADPOS } \\
\text { System for Dose and Position Quality Assurance of 4D CyberKnife } \\
\text { Treatments. Ottawa, Canada, 2015 }\end{array}$ \\
\hline WC 2015 & $\begin{array}{l}\text { Marants, R., Vandervoort, E., Cygler, J. E. Dose and Position Quality } \\
\text { Assurance using the RADPOS System for 4D Radiotherapy with } \\
\text { CyberKnife. World Congress on Medical Physics and Biomedical } \\
\text { Engineering, June 7-12, 2015, Toronto, Canada. IFMBE Proceedings 51, } \\
599-602,2015 \\
\text { AAPM 2015 }\end{array}$ \\
\hline $\begin{array}{l}\text { Marants, R., Vandervoort, E., Cygler, J. E. Quality Assurance using the } \\
\text { RADOS System for 4D Radiotherapy with CyberKnife. Med Phys 42, }\end{array}$ \\
\hline
\end{tabular}




\section{Table of Contents}

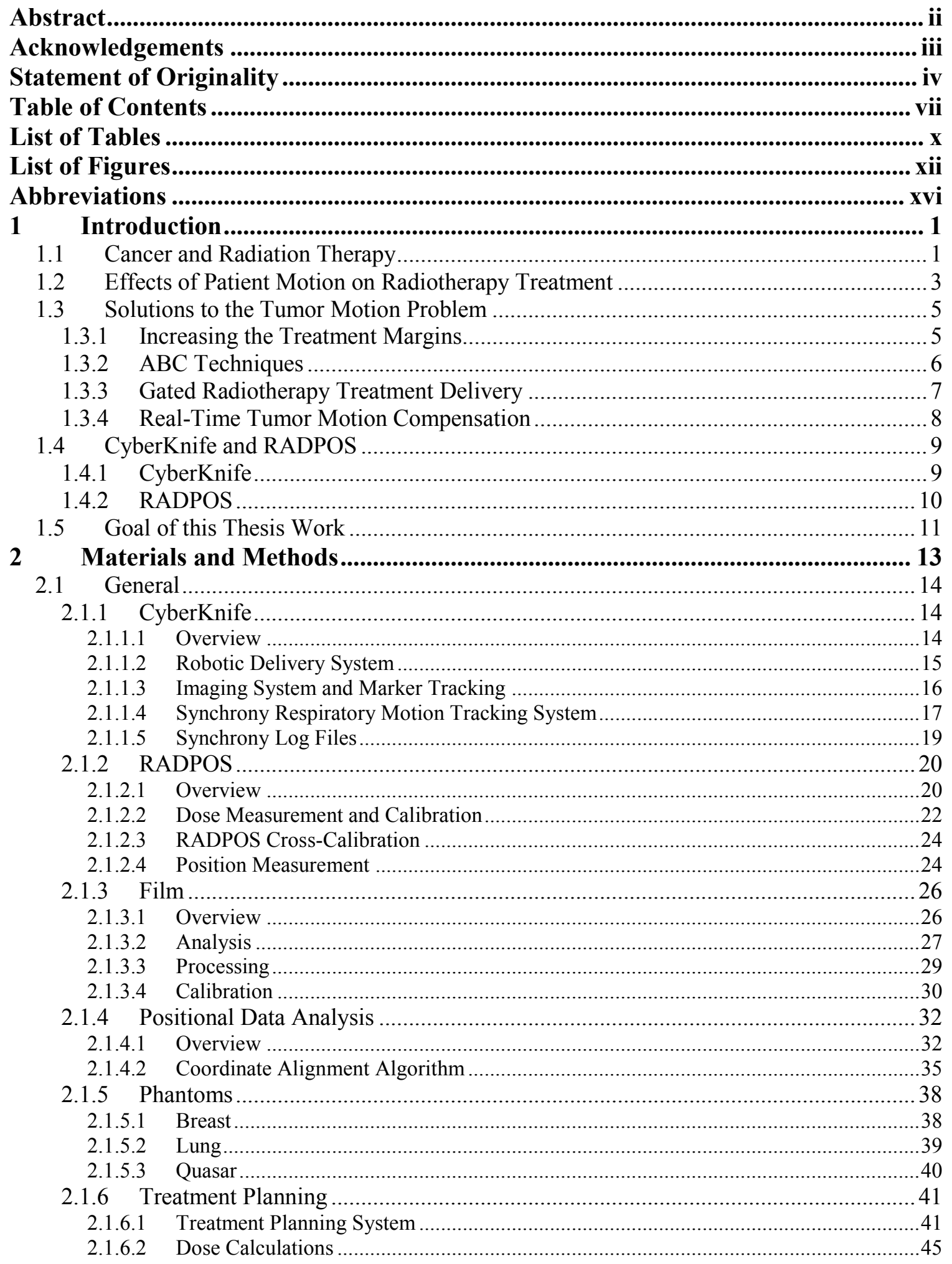




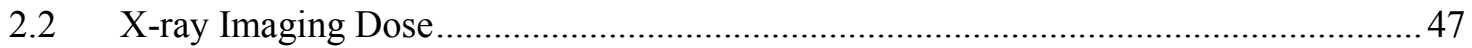

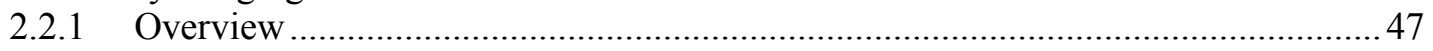

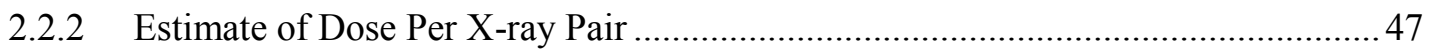

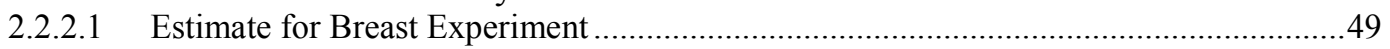

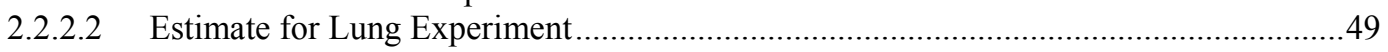

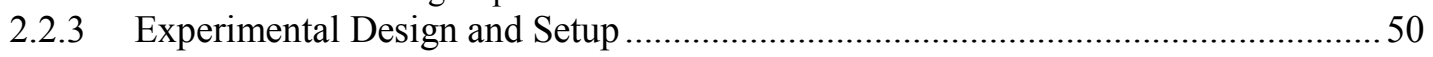

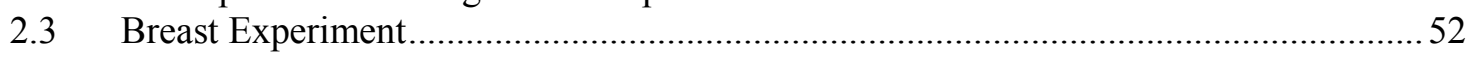

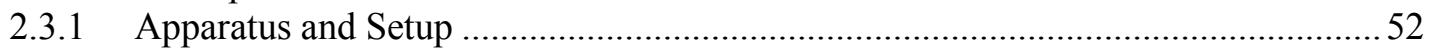

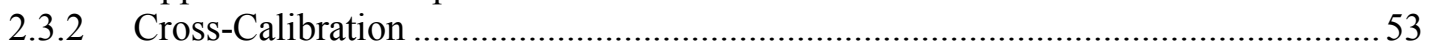

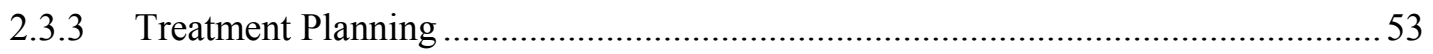

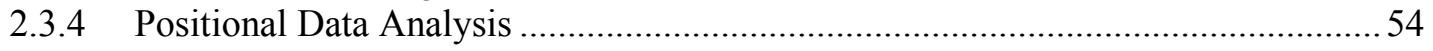

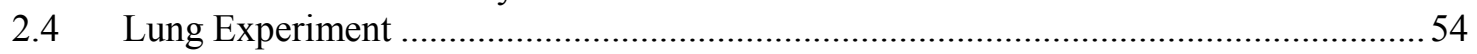

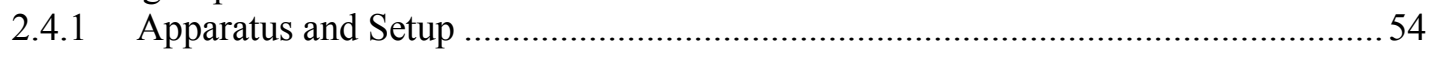

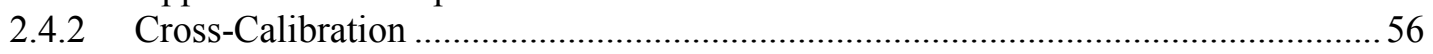

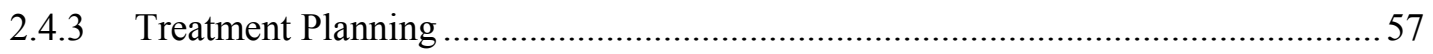

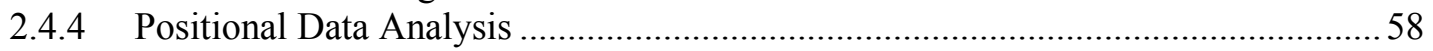

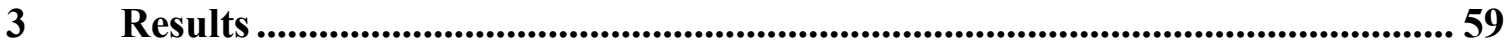

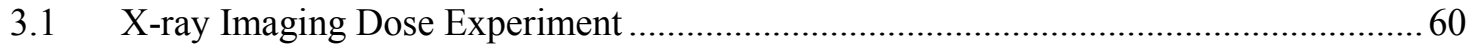

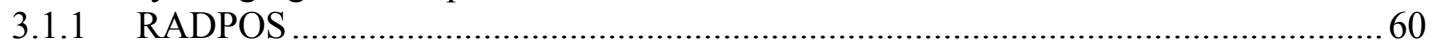

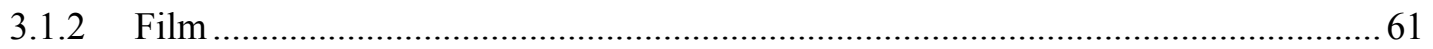

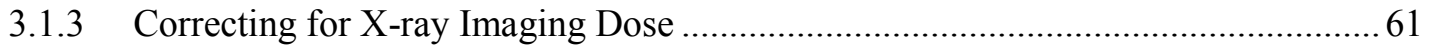

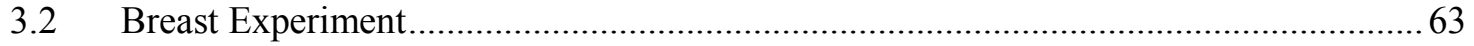

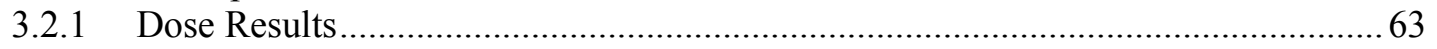

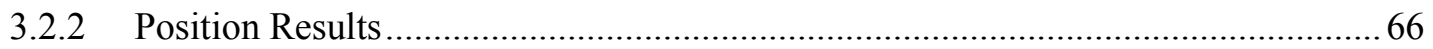

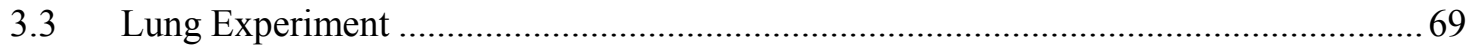

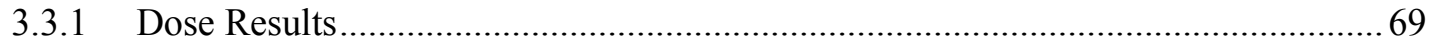

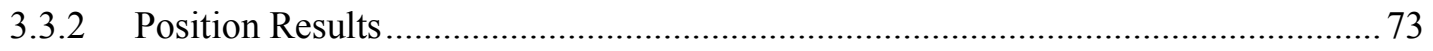

4 Discussion.................................................................................................................... 75

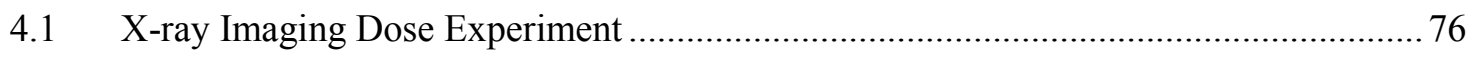

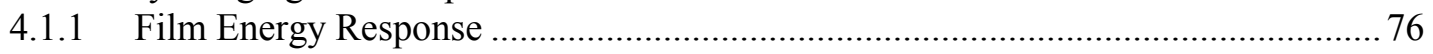

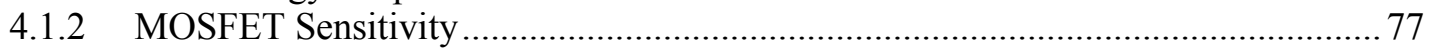

4.1.3 Estimate versus Measurement of Dose Per X-ray Pair ........................................... 79

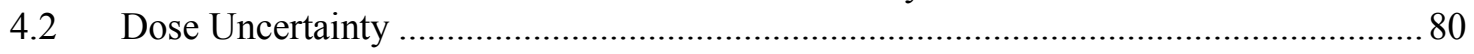

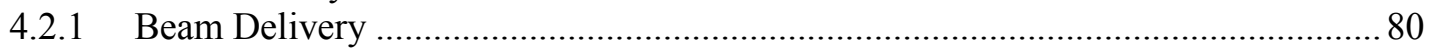

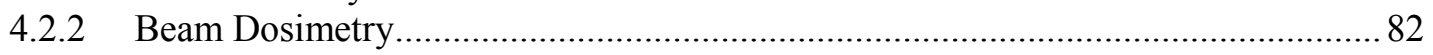

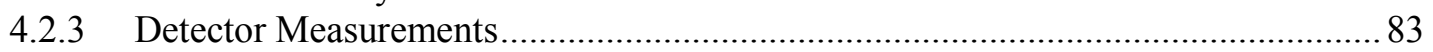

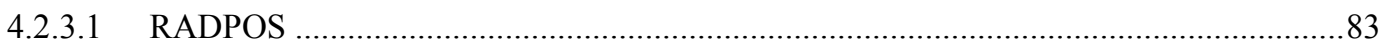

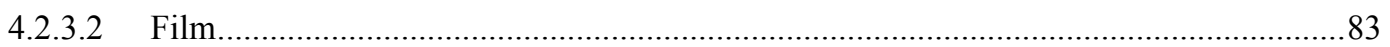

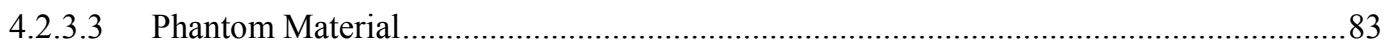

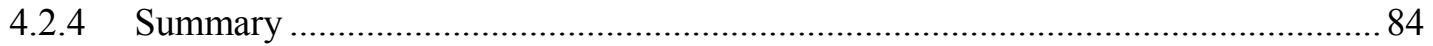

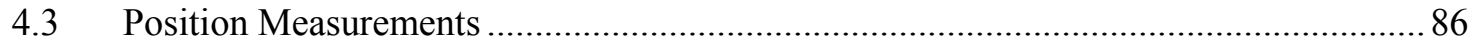

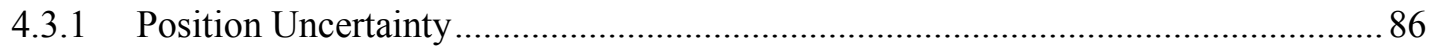

4.3.2 Position Data Comparison............................................................. 88

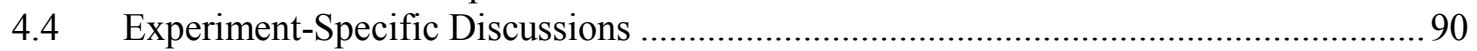

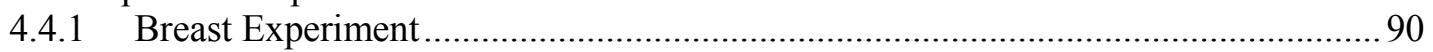

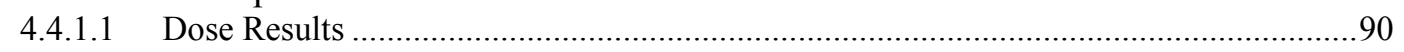

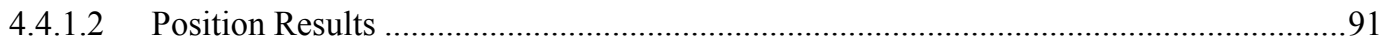

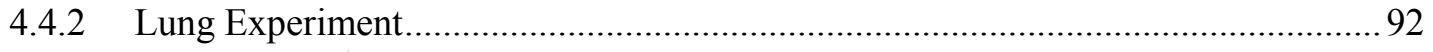

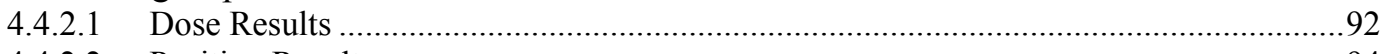

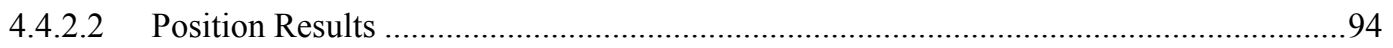

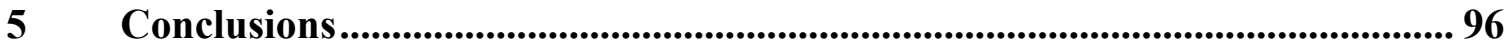




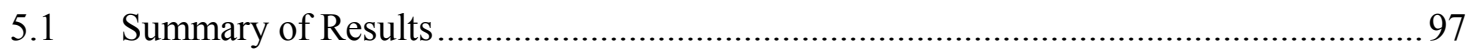

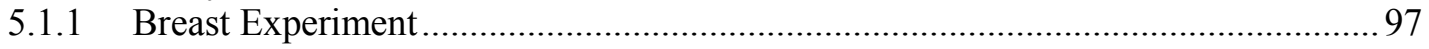

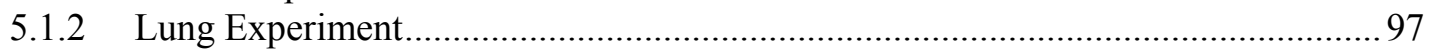

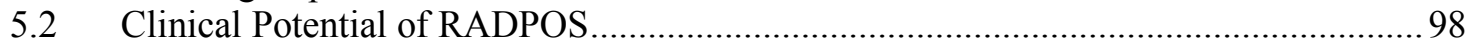

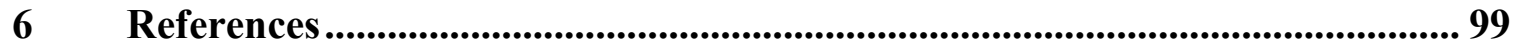




\section{List of Tables}

Table 2.1 Materials making up the breast phantom and Quasar-lung insert setup, along with their associated mass densities and mass attenuation coefficients for an energy of 27 $\mathrm{keV}$ (approximately $1 / 3$ of $80 \mathrm{kVp}$ ) and $40 \mathrm{keV}$ (approximately $1 / 3$ of $130 \mathrm{kVp}$ )............ 48

Table 2.2 Surface dose per single image (from CyberKnife white paper [49]). In addition, approximate unattenuated dose per x-ray values, based on x-ray parameters used during the breast and lung experiments, are given in italics.

Table 2.3 Summary of the main details for the four breast experiment treatment cases. . 53

Table 2.4 Summary of the main details for the five lung experiment treatment cases..... 55

Table 3.1 Details and parameters for $\mathrm{x}$-ray imaging dose experiment. ........................... 60

Table 3.2 Summary of $\mathrm{x}$-ray imaging dose experiment results.

Table 3.3 Change in film dose measurements corrected for $x$-ray imaging dose during the breast experiment.

Table 3.4 Change in RADPOS voltage measurements corrected for x-ray imaging dose during the lung experiment.

Table 3.5 Change in film dose measurements corrected for x-ray imaging dose during the lung experiment.

Table 3.6 Dose comparison between TPS, film (corrected for imaging x-ray dose), and RADPOS, as well as the percent of film pixels passing a 3\%/1 $\mathrm{mm}$ gamma index criteria.

Table 3.7 Percent difference comparisons between TPS, film, and RADPOS dose values.

Table 3.8 Quantitative positional results for the three dynamic treatment cases of the breast, comparing the LED, RADPOS, fiducial and model positional data. The primary direction of motion for all experimental cases is the $\mathrm{z}$ direction. Comparison 1 was performed in the fiducial time domain, whereas Comparison 2 was performed in either the RADPOS (LED-RADPOS, RADPOS-Model) or Synchrony (LED-MODEL) time domains (see section 2.1.4.2).

Table 3.9 TPS dose values at different phases of the respiratory cycle in the RADPOS plane and film plane.

Table 3.10 Measured RADPOS and film (both corrected for imaging x-ray dose) dose values, as well as percent difference comparisons between RADPOS and mid respiration

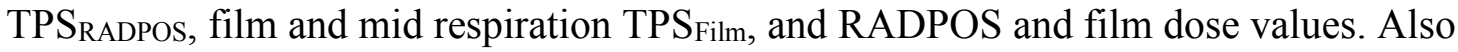
shown is the percent of film pixels passing a $3 \% / 1 \mathrm{~mm}$ and $3 \% / 2 \mathrm{~mm}$ gamma index criteria.

Table 3.11 Positional analysis results for the three dynamic treatment cases of the lung experiment, comparing the RADPOS and model positional data. The primary direction of 
motion for all experimental cases is the $\mathrm{x}$ direction. Comparison 1 was performed in the fiducial time domain, whereas Comparison 2 was performed in either the RADPOS (LED-RADPOS, RADPOS-Model) or Synchrony (LED-MODEL) time domains (see section 2.1.4.2)

Table 4.1 Summary of the various sources of dosimetric uncertainty, along with the associated uncertainty evaluation type.

Table 4.2 Summary of the various contributions to the overall positional uncertainty for the general case of motion along a single direction. The uncertainties along the primary and non-primary directions of motion are given, along with how the uncertainties change with and without RADPOS.. 88 


\section{List of Figures}

Figure 1.1 Canadian cancer incidence for males (a) and females (b) from 1985 to those estimated for 2014 based on the aging population (top curve), population growth (middle curve), and changes in cancer risk and cancer control practices. Obtained with permission from Canadian Statistics 2014 [1].

Figure 1.2 CT scan of the same patient showing instances of taking (a) and not taking (b) respiratory-induced motion into account using gating. The gross tumor volume, GTV, has been contoured in both cases. Obtained with permission from Keall et al., 2002 [16]...... 4

Figure 1.3 A moving target being irradiated with a static beam. Parts of the target move into and out of the primary radiation beam, resulting in the need for increased margins. Adapted from Keall et al., 2001 [17].

Figure 1.4 The typical delineations of the GTV, CTV, and PTV, along with the treated volume and irradiated volume, as given in ICRU Report 62. Adapted from ICRU Report $62[18]$.

Figure 1.5 A modified ventilator used to control patient breathing. Obtained with permission from Wong et al., 1999 [21]......

Figure 1.6 The Varian RPM system. The tracking camera (visible on the right) emits infrared light that is reflected back by the marker block (visible on the left) and used for motion tracking. Obtained with permission from Suhy and Maniawski, 2011 [24].

Figure 1.7 The RADPOS dosimeter probe. The MOSFET and position sensor are shown, separated by $8 \mathrm{~mm}$. 10

Figure 2.1 CyberKnife treatment room at the Ottawa Hospital Cancer Centre. The robotic delivery system and imaging system are shown, along with their associated components.

Figure 2.2 Schematic of nodes (x-marks) and paths (connecting lines) surrounding the patient couch of the CyberKnife. The imaging isocenter is represented by the solid circle. Adapted from Dieterich el al., 2011 [28].

Figure 2.3 Layout of CyberKnife's x-ray imaging system. The x-ray sources are mounted to the ceiling orthogonal to one another, and their associated image receptors are located in the floor. Adapted from Dieterich el al., 2011 [28].

Figure 2.4 RADPOS 4D dosimetry system. An overview of RADPOS' components, adapted from Cherpak el al., 2009 [30], is displayed in (a), while a closer view of the dosimeter probe is shown in (b).

Figure 2.5 MOSFET dosimeter, which is part of the RADPOS probe. Adapted from Soubra et al., 1994 [25].

Figure 2.6 Change in the MOSFET's threshold voltage due to ionizing radiation. Adapted from Soubra et al., 1994 [25]. 
Figure 2.7 The amplitude profile of the magnetic field produced by the DC magnetic field transmitter has a trapezoidal shape.

Figure 2.8 RADPOS' DC magnetic field transmitter. Also shown is its coordinate system.

Figure 2.9 Scan settings (a) and adjustments (b) used for EBT3 GafChromic film processing.

Figure 2.10 Film arrangements and sizes used for pre- and post-experiment analysis. Shown are the films before (a) and after (b) irradiation, along with the sizes of each film piece.

Figure 2.11 Film arrangements and sizes used for pre- and post-experiment calibration. Shown are the films (a) before and (b) after irradiation, along with the sizes of each film piece.

Figure 2.12 Example of a how the c parameter (i.e., calibration curve exponent) is determined. The value of $\mathrm{c}$ which minimizes the error function is used for the calibration fit. In this case, a value of $c=1.3$ optimizes the associated netOD versus dose calibration curve fit.

Figure 2.13 Shown are the (a) LED and (b) RADPOS motion signal waveforms. A common feature from both plots, such as the "jump" indicated by the black arrow, was used to temporally align the waveforms (which appear at a different time due to differences in how the temporal origin is defined in the two systems).

Figure 2.14 3D renderings of semi-spherical Solid Water breast phantom. Both halves (a) and one half (b) of the phantom is shown, along with approximate positions of fiducials and detectors. 38

Figure 2.15 3D renderings of cylindrical cedar wood lung insert. Both halves (a) and one half $(b, c, d)$ of the phantom is shown, along with other features common to all experiments.

Figure 2.16 The Quasar Respiratory Motion Phantom (a) hardware and (b) software. Obtained with permission from Modus Medical Devices Inc.

Figure 2.17 Screen capture of the MultiPlan treatment planning system's fuse step. In this case, a fusion of the CT image for the Quasar-breast phantom system with a small field of view, high resolution CT image of just the breast phantom is shown. 41

Figure 2.18 Screen capture of the MultiPlan treatment planning system's contour step. The MOSFET and position sensor of the RADPOS detector have been outlined............ 42

Figure 2.19 Screen capture of the MultiPlan treatment planning system's align step. The treatment parameters are being set and the locations of the fiducials have been identified.

Figure 2.20 Screen capture of the MultiPlan treatment planning system's plan step. The spatial distribution of treatment beams are shown in the top-left and the dose distribution within the breast phantom is shown in the bottom-left. 
Figure 2.21 CT image of medial slice through the breast phantom. Shown is the measured thickness of the Solid Water material through which the imaging X-rays must pass through to reach the RADPOS MOSFET dosimeter.

Figure 2.22 CT image of medial slice through the Quasar. Shown are the measured thicknesses of the various materials through which the imaging $\mathrm{X}$-rays must pass through to reach the RADPOS MOSFET dosimeter.

Figure 2.23 Cross-calibration conditions used for the (a) ion chamber readings, (b) RADPOS calibration, and (c) film calibration during the x-ray imaging dose experiment.

Figure 2.24 Apparatus and setup for the breast experiment. 52

Figure 2.25 Setups for the dynamic cases of the lung experiment. For case (a) I1, the lung insert and chest platform (with the LEDs) both move together. For cases (b) I2 and P3, one Quasar has the insert and the other has the chest platform, which are made to move separately.

Figure 2.26 Illustration showing how the source positions (black circles) and beam directions (black lines) change for a moving target on the CyberKnife system. The middle (a) of respiration, as well as the maximum (b) superior and (c) inferior extents of Quasar respiratory motion are shown. In each case, the positions of the nodes remain constant but the direction the LINAC is pointing follows the Solid Water tumor within the lung insert.

Figure 3.1 Sagittal dose planes within the moving breast phantom for (a) MultiPlan TPS and (b) the average film data, as well as (c) 2D gamma comparison for 3\%/1 mm, which shows pixels which do not pass the gamma criteria as black. Also shown is a dose profile comparison along the directions: (d) superior/inferior, and (e) anterior/posterior (direction of phantom motion).

Figure 3.2 The LEDs, RADPOS, and fiducial position measurements along the $\mathrm{x}, \mathrm{y}$, and $\mathrm{z}$ directions after implementation of the algorithm.... 66

Figure 3.3 Closer view of a region of Figure 3.2, showing how the three position tracking modalities match up at the fiducial's time points.

Figure 3.4 Illustration of cross-section through the lung insert, showing the film (gray bar) and RADPOS (black circle) positions inside of it. Also shown are the coronal planes for the film and RADPOS, which were used for TPS dose calculations. 69

Figure 3.5 Dose profiles along the coronal plane within the lung insert (case I1) for (a) MultiPlan TPS and (b) the average film data, as well as (c) 2D gamma comparison for $3 \% / 1 \mathrm{~mm}$, which shows pixels which do not pass the gamma criteria as black. Also shown is a dose profile comparison along the directions: (d) superior/inferior (direction of phantom motion), and (e) left/right.....

Figure 3.6 Dose profiles along the coronal plane within the lung insert (case P0) for (a) MultiPlan TPS and (b) the average film data, as well as (c) 2D gamma comparison for $3 \% / 2 \mathrm{~mm}$, which shows pixels which do not pass the gamma criteria as black. Also shown is a dose profile comparison along the directions: (d) superior/inferior (direction of phantom motion), and (e) left/right.... 
Figure 3.7 Comparison of positional data for the RADPOS position sensor (solid gray curve), Synchrony predictive correlation model (dashed black curve), and x-ray imaged fiducial markers (black dots) after implementation of the coordinate alignment algorithm. The results are shown for treatment cases (a) I1, (b) I2, and (c) P1. The data presented is for the primary direction of motion, as motion along the other directions was small $(<1$ $\mathrm{mm}$ peak-to-peak amplitude). The time axes represent the time elapsed since the last program start of the Synchrony system.

Figure 4.1 Response of MOSFET dosimeter as a function of photon energy. A significant over-response is observed at lower photon energies. Obtained with permission from Wang et al., 2005 [58].

Figure 4.2 Illustration of the main differences between (a) isocentric and (b) nonisocentric treatment deliveries on the CyberKnife system. In both cases, the OAR, tumor, and treatment beams are represented by the light gray oval, dark gray irregularly-shaped object, and transparent lines, respectively. In the case of isocentric delivery, the beams intersecting at a common point (isocenter, red dot) pass through the OAR (indicated by the black arrows). For this fictitious example, the OAR is completely spared of entrance and exit dose in the case of non-isocentric delivery. 


\section{Abbreviations}

\begin{tabular}{|c|c|}
\hline $\mathbf{A B C}$ & active breathing control \\
\hline CT & computed tomography \\
\hline CTG & certified therapy grade \\
\hline CTV & clinical target volume \\
\hline DIBH & deep inspiration breathe hold \\
\hline DVH & dose volume histogram \\
\hline GTV & gross tumor volume \\
\hline LED & light emitting diode \\
\hline LINAC & linear accelerator \\
\hline MOSFET & metal-oxide semiconductor field-effect transistor \\
\hline NTCP & normal tissue complication probability \\
\hline OAR & organs at risk \\
\hline PTV & planning target volume \\
\hline QUASAR & Quality Assurance System for Advanced Radiology and Radiotherapy \\
\hline RADPOS & Radiation Positioning System \\
\hline ROI & region of interest \\
\hline TPS & treatment planning \\
\hline
\end{tabular}




\section{Introduction}

\subsection{Cancer and Radiation Therapy}

The Canadian Cancer Society reports that 2 in 5 Canadians will be afflicted with cancer sometime during their lifetime, and nearly 200,000 new cases of cancer were expected to be diagnosed in 2014 [1], as shown in Figure 1.1. In addition to surgery and chemotherapy, radiation therapy is a well-developed and effective cancer treatment approach. Over half of all cancer patients will receive some form of radiation therapy throughout the course of their treatment [2].

The primary goal of radiation therapy is to maximize the amount of dose received by an anatomical target (e.g., a malignant tumor), while at the same time minimizing the amount of dose received by surrounding healthy tissue (i.e., organs at risk, OAR), in

order to achieve curative and/or palliative results. On a microscopic level, ionizing radiation destroys cells primarily via DNA double strand breaks [3]. As a result, cells are no longer able to replicate, which halts their proliferation and keeps the targeted tissue from growing. 
(a)

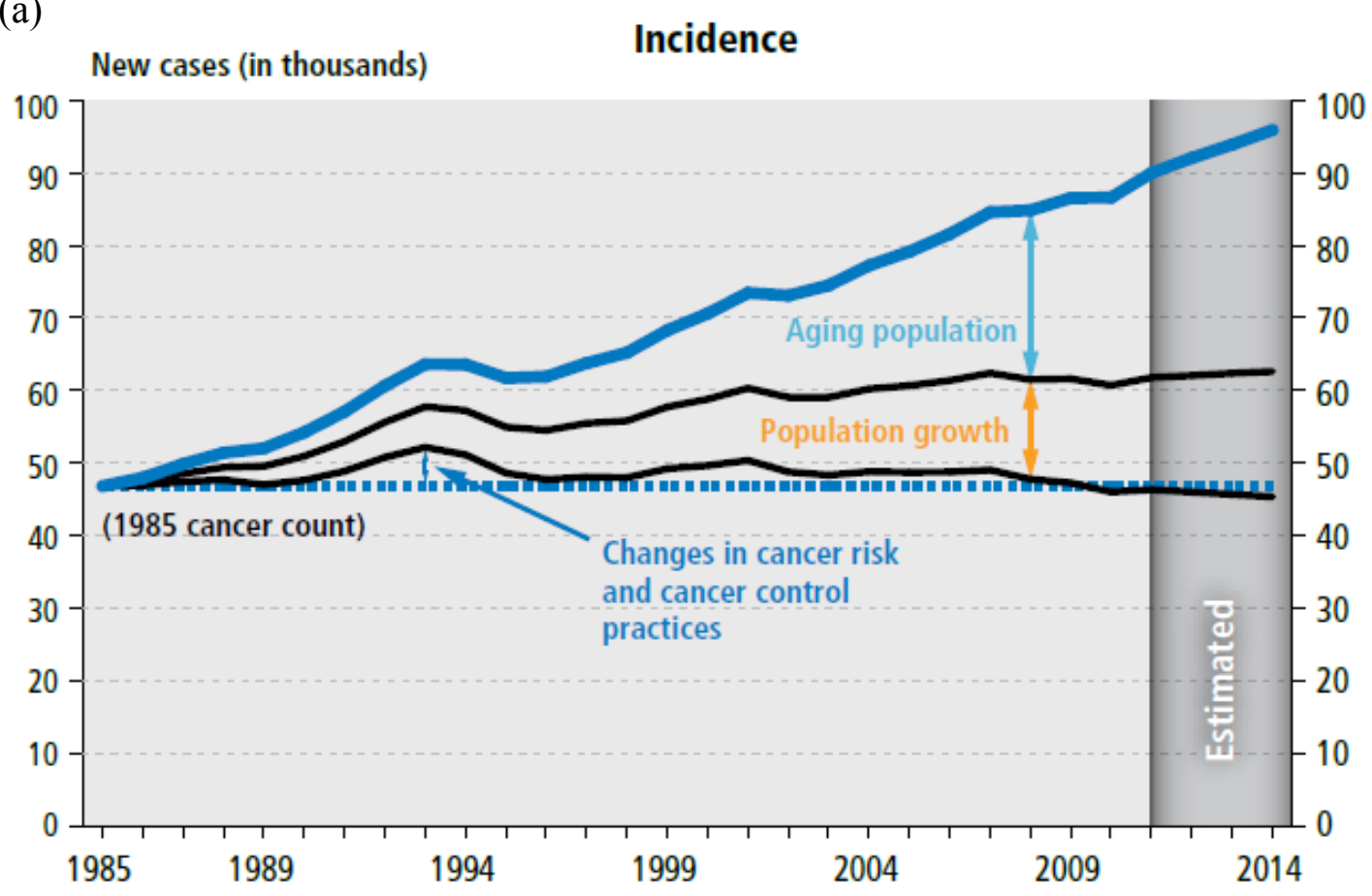

(b)

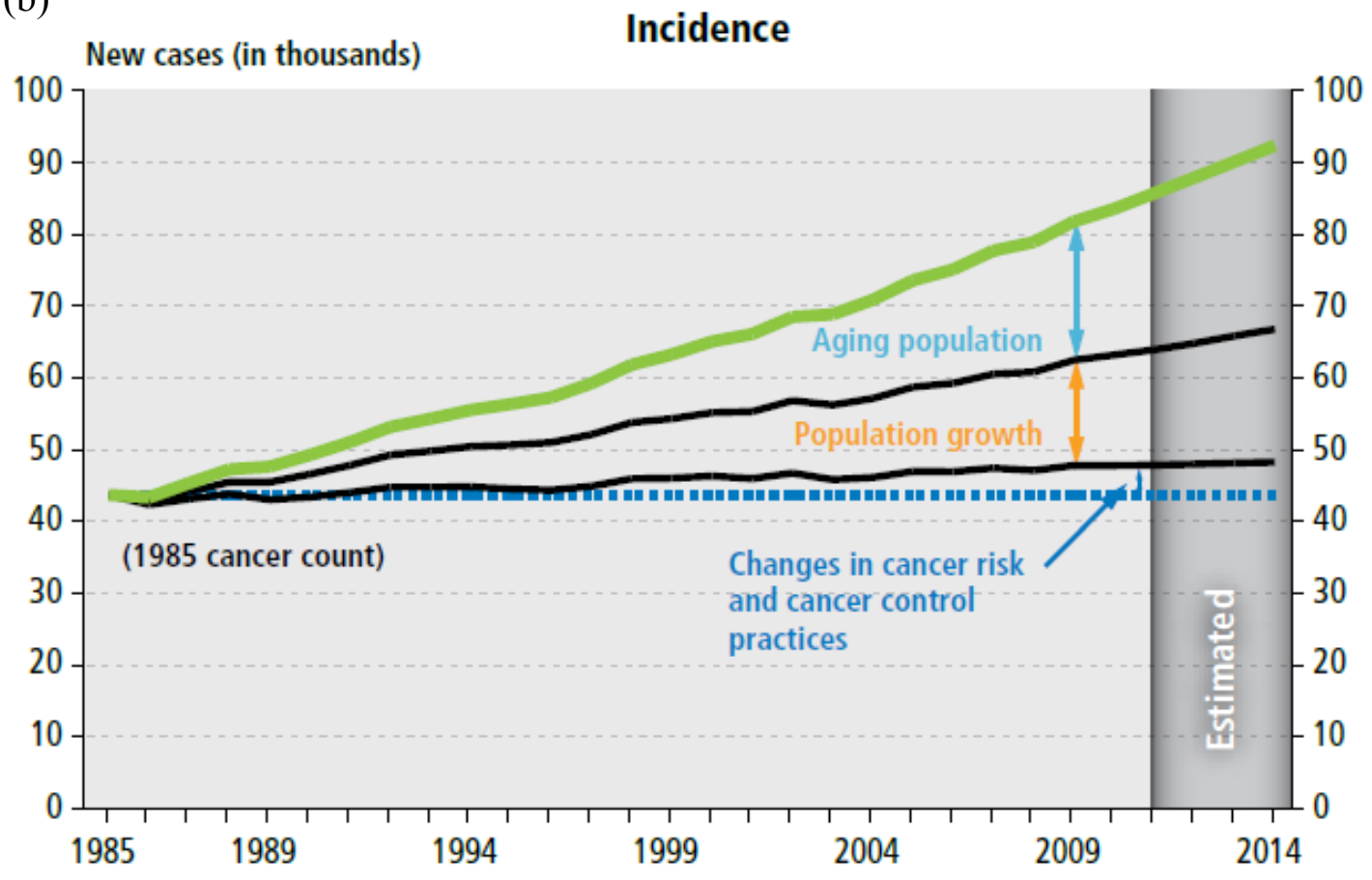

Figure 1.1 Canadian cancer incidence for males (a) and females (b) from 1985 to those estimated for 2014 based on the aging population (top curve), population growth (middle curve), and changes in cancer risk and cancer control practices. Obtained with permission from Canadian Statistics 2014 [1]. 


\subsection{Effects of Patient Motion on Radiotherapy Treatment}

Radiotherapy treatment becomes more challenging in cases where patient motion, such as breathing, causes movement of the treatment target or OAR. De Groote et al. [4] have found that chest wall motion is on the order of $4 \mathrm{~mm}$ during tidal breathing, and a number of studies have shown that lung tumor motion can range from 5 to $12 \mathrm{~mm}$ during respiration [5-8]. This can lead to serious issues throughout the various stages of radiotherapy treatment.

During a CT scan for treatment planning, respiration-induced organ and/or tumor motion may lead to motion artifacts and geometric distortions [9], degrading the quality of x-ray images taken and compromising the quality of a patient's radiotherapy treatment. An example of this is shown in Figure 1.2. Identifying and contouring critical structures also becomes difficult, and the expansion of the clinical target volume, CTV, to planning target volume, PTV, may end up being inaccurate, excessive, or both. Balter et al. [10] have shown that radiation path length, organ position and volume, and dose evaluation metrics (such as dose-volume histograms and normal tissue complication probability) will change because of patient free-breathing, as opposed to maintaining breath-hold at inhalation or exhalation, established during CT acquisition. Finally, during treatment delivery, target motion may result in dose blurring and the irradiation of healthy or sensitive tissue, or missing of the target partially or completely [11]. This may lead to a change in the dose delivered to the target on the order of several percent [12-14].

Due to the aforementioned concerns, taking respiratory-induced motion into account is not a trivial issue, but an essential consideration. Fortunately, several methods 
to deal with this problem at different stages of radiotherapy treatment have been explored, developed, and are currently in use [15].

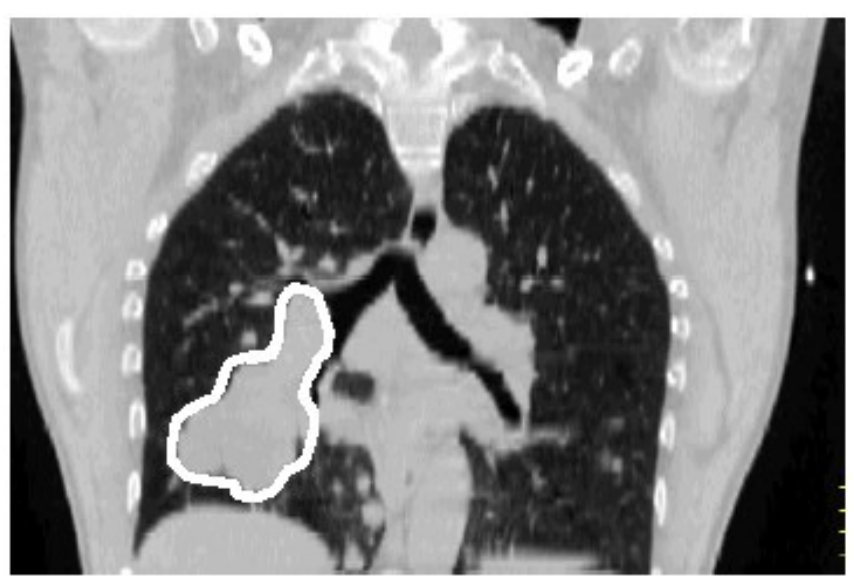

(a)

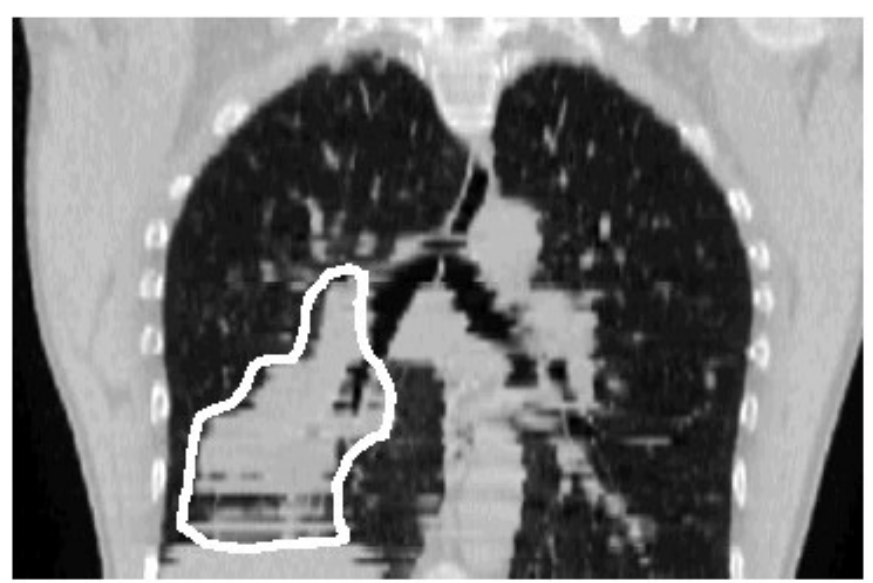

(b)

Figure 1.2 CT scan of the same patient showing instances of taking (a) and not taking (b) respiratoryinduced motion into account using gating. The gross tumor volume, GTV, has been contoured in both cases. Obtained with permission from Keall et al., 2002 [16].

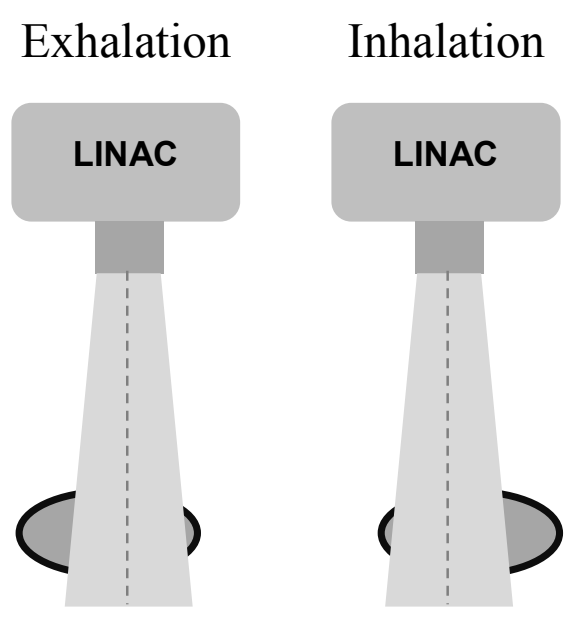

Figure 1.3 A moving target being irradiated with a static beam. Parts of the target move into and out of the primary radiation beam, resulting in the need for increased margins. Adapted from Keall $e t$ al., 2001 [17]. 


\subsection{Solutions to the Tumor Motion Problem}

\subsubsection{Increasing the Treatment Margins}

One way to account for tumor motion due to voluntary and/or involuntary bodily functions is to expand the margins of a target in order to encompass the tumor's full trajectory of movement. Figure 1.3 shows how target motion may lead to a need for larger treatment margins. More specifically, once the GTV and CTV have been delineated by the physician, the PTV is then also added, as seen in Figure 1.4. The PTV is meant to factor in geometric variations of the radiation field and physiological movements (e.g., breathing, heartbeat, etc.), as well as uncertainties associated with positioning of the patient and alignment of treatment beams before and during treatment [18]. Although this allows the tumor to always remain within the primary radiation beam during treatment and receive the prescribed dose, more healthy tissue is irradiated due to the increased margins around the tumor [19]. If nearby healthy tissue is particularly radiosensitive, this may limit the amount of dose prescribed to the tumor and result in compromised treatment outcomes.

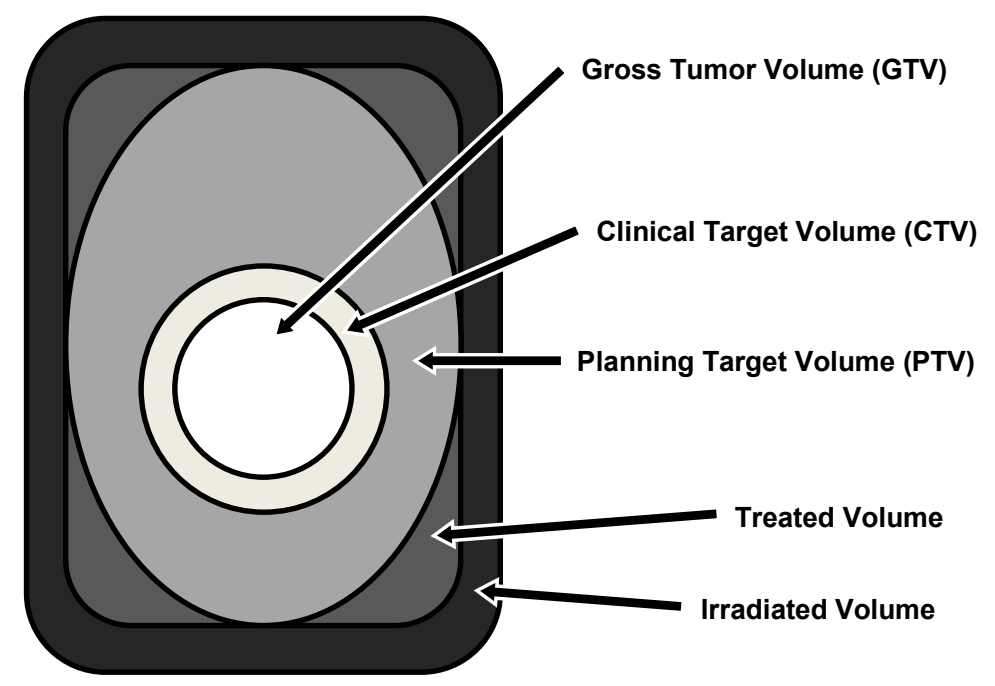

Figure 1.4 The typical delineations of the GTV, CTV, and PTV, along with the treated volume and irradiated volume, as given in ICRU Report 62. Adapted from ICRU Report 62 [18]. 


\subsubsection{ABC Techniques}

Another solution is to implement breath-hold techniques or other active breathing control, ABC, approaches. Adapting the way a patient is breathing may help limit the amount of normal tissue irradiation and improve treatment of the target region. Deepinspiration breath-hold, DIBH, techniques minimize tumor motion due to breathing, as well as expand the lungs to move healthy tissue out of the primary radiation beam [20]. Other ABC methods involve verbally coaching the patient to breathe in a specified fashion, or using specialized apparatus, as seen in Figure 1.5, which limits airflow and/or physically constrains anatomical movement, such as expansion of the thorax or abdomen. $\mathrm{ABC}$ approaches generally result in high intra-fraction precision and reproducibility, but cannot reliably be applied to all patients [21].

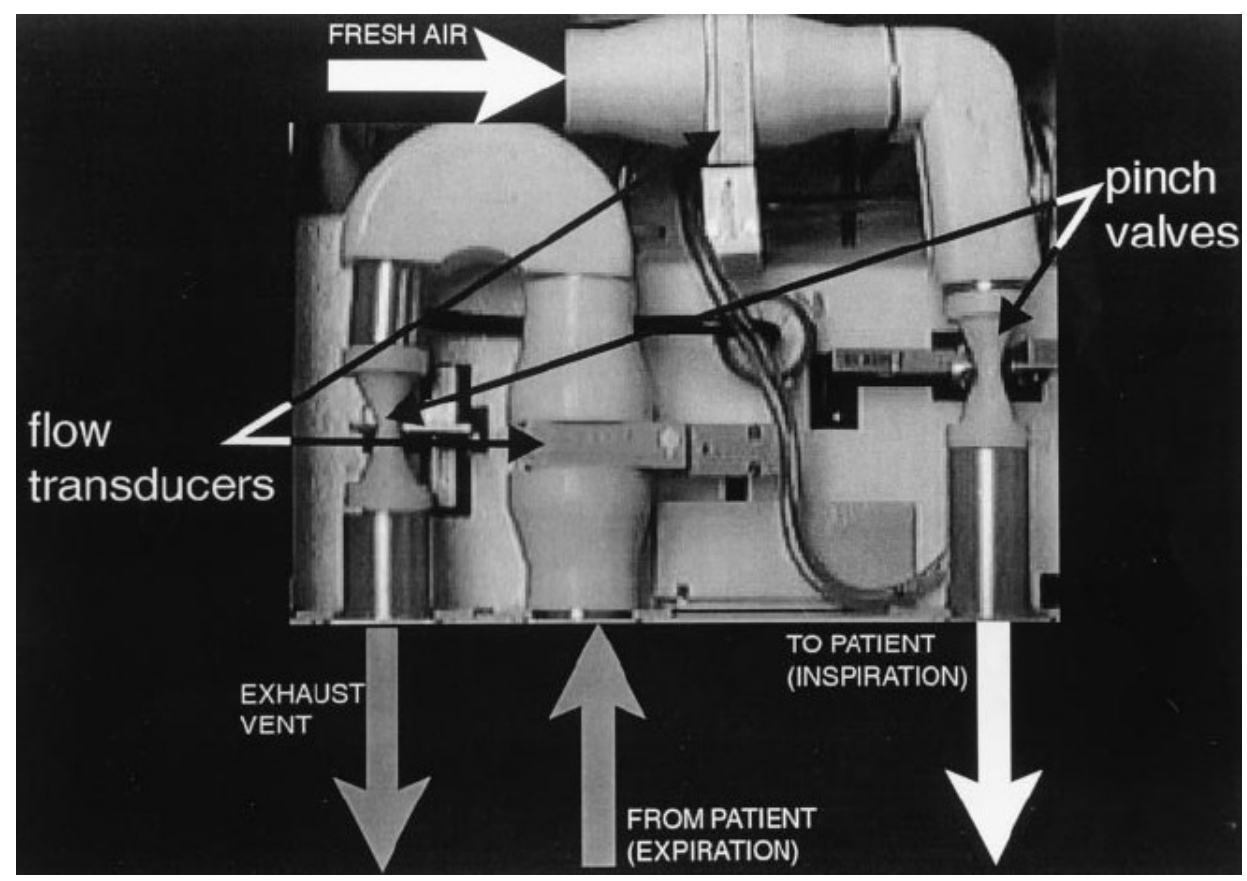

Figure 1.5 A modified ventilator used to control patient breathing. Obtained with permission from Wong et al., 1999 [21]. 


\subsubsection{Gated Radiotherapy Treatment Delivery}

An additional approach that can account for organ motion due to breathing is gated radiation treatment delivery. Gated radiotherapy involves the selective delivery of radiation to a moving target at a time during which the target is known to be within a certain portion of the respiration cycle. Radiotherapy with respiratory gating allows for higher dose delivery to the target while minimizing additional dose to surrounding healthy tissue because of the reduced treatment margin [22]. To be implemented effectively, gating relies on identifying the position and overall trajectory of the tumor target with a high degree of accuracy. This is most commonly accomplished by tracking the motion of internal or external target motion surrogates. The Varian Real-Time Position Management (RPM) system, which performs external motion tracking used to set gating thresholds, is pictured in Figure 1.6.

Respiratory gating leads to a reduced treatment machine duty cycle, resulting in longer treatment times and a decrease in daily patient throughput [16, 23]. Also, residual target motion may take place during the gating interval, leading to some healthy tissue irradiation and the possibility of a geographic miss of the tumor.

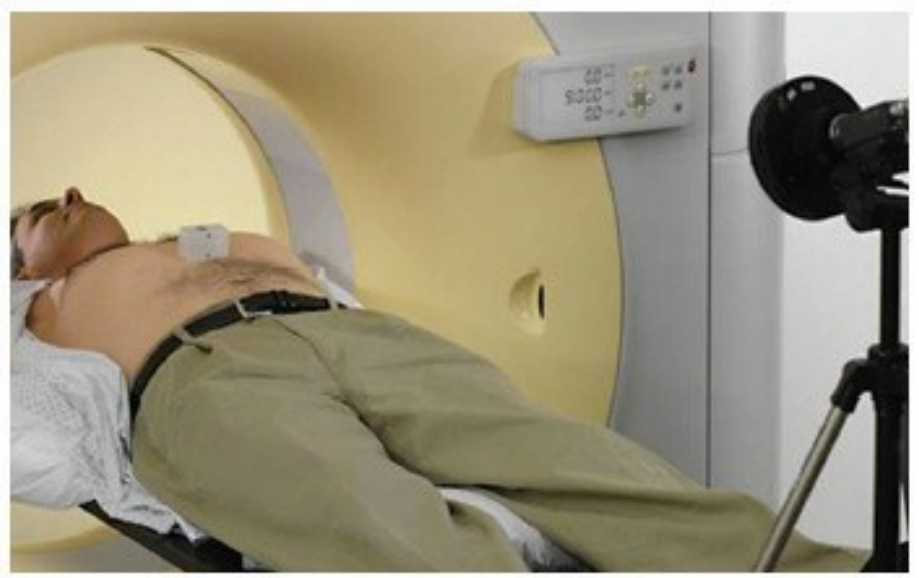

Figure 1.6 The Varian RPM system. The tracking camera (visible on the right) emits infrared light that is reflected back by the marker block (visible on the left) and used for motion tracking. Obtained with permission from Suhy and Maniawski, 2011 [24]. 


\subsubsection{Real-Time Tumor Motion Compensation}

For successful implementation, radiation therapy with real-time tumor motion tracking has to determine the target location, predict its motion in order to account for time delays in hardware and software, and adjust the radiation beam position [25]. Similar to gated radiotherapy, real-time compensation relies on following the movement of the target in order to spare healthy tissue during beam delivery. This can be done by imaging the target directly, following the movement of a target motion surrogate, tracking the positions of internally implanted radiopaque fiducial markers, and using other position detection technology, such as electromagnetic positioning sensors [15]. As opposed to gating, real-time compensation dynamically shifts the position of the radiation beam relative to the target in order to match its motion. This is typically accomplished either by repositioning the LINAC's multi-leaf collimator aperture or by changing the direction of the radiation beam source using a robotic manipulator. This allows for a reduced treatment margin, similarly to gating, but also has a higher duty cycle, allowing for more efficient treatments in terms of dose delivery and patient throughput.

The commercially-available CyberKnife radiosurgery treatment delivery system makes use of real-time tumor motion compensation. A mobile LINAC is dynamically repositioned during treatment, based on internal and external target tracking, in order to compensate for tumor motion. 


\subsection{CyberKnife and RADPOS}

\subsubsection{CyberKnife}

The Accuray CyberKnife Robotic Radiosurgery System consists of a compact linear accelerator mounted on a robotic arm, which gives it six degrees-of-freedom of motion. It is able to deliver small $x$-ray radiotherapy beams to the tumor from many different non-coplanar directions, resulting in highly conformal dose distributions. In addition, the CyberKnife system implements tumor tracking through the use of internally implanted metal fiducials and motion tracking through the use of external LED optical markers [26].

Using the LED and fiducial position measurements, CyberKnife's Synchrony Respiratory Motion Tracking System builds a predictive correlation model. This model is used to adapt beam delivery while the radiation beam is on, in order to account for a patient's breathing pattern. As the treatment progresses, the model is updated with the most recent position tracking data, adjusting for any variations in the breathing pattern of the patient.

By combining frameless LINAC movement with real-time tumor motion tracking and compensation, the CyberKnife radiosurgery treatment delivery system offers a unique and effective solution to tumor motion during radiotherapy. But with such a complex delivery system, there is a need for thorough quality assurance which can look independently at different components of this system. This can potentially be accomplished with the use of RADPOS. 


\subsubsection{RADPOS}

The RADPOS 4D dosimetry system consists of a probe which combines a small active volume MOSFET (metal-oxide-semiconductor-field-effect transistor) dosimeter, or microMOSFET, and an electromagnetic positioning sensor. RADPOS has the ability to perform real-time dose and position measurements simultaneously, making it an excellent candidate for acting as an independent quality assurance tool for the dose delivery and position tracking of the CyberKnife system.

The microMOSFET and position sensor are separated by $8 \mathrm{~mm}$ to limit radiation attenuation and disturbance of the particle fluence near the dosimeter, as seen in Figure 1.7. In order to perform dose measurements, RADPOS' microMOSFET dosimeter measures a threshold voltage difference which is induced when the microMOSFET is irradiated. It is this threshold voltage difference which is proportional to the absorbed dose [27]. The position measurements are obtained using a DC magnetic field transmitter which emits a pulsed 3D magnetic field that is detected and used by the RADPOS electromagnetic positioning sensor to determine its position coordinates.

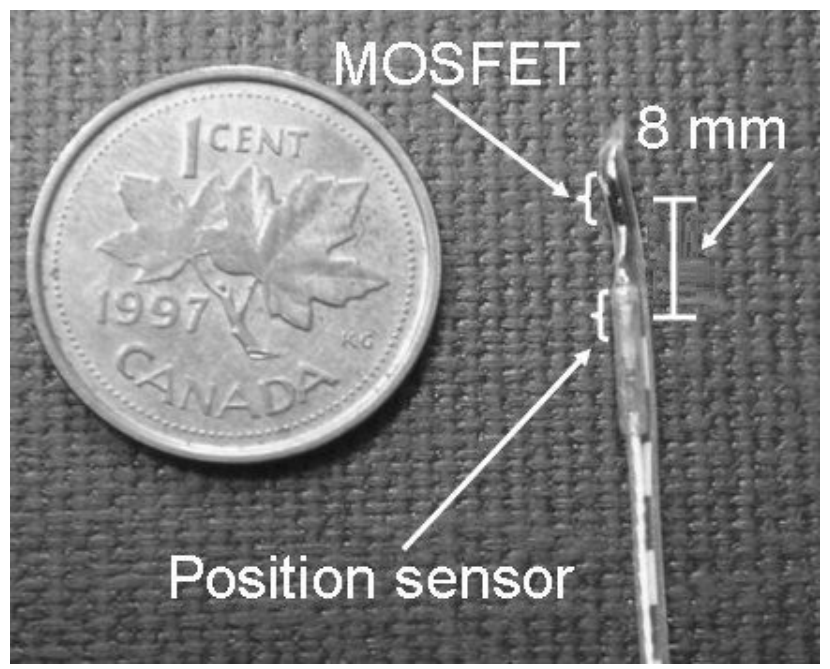

Figure 1.7 The RADPOS dosimeter probe. The MOSFET and position sensor are shown, separated by $8 \mathrm{~mm}$. 


\subsection{Goal of this Thesis Work}

The goal of this project is to quantitatively demonstrate RADPOS' effectiveness as a dose and position quality assurance tool for radiotherapy with CyberKnife. A number of QA tests and protocols outlined in the report of AAPM TG-135, quality assurance for robotic radiosurgery [28], could be addressed using RADPOS as an independent performance verification in addition to the standard set of QA measurement tools and procedures. The project goal will be accomplished by addressing the following experiment-based questions:

1. Can RADPOS be used as an accurate and precise CyberKnife radiotherapy treatment quality assurance tool? RADPOS could be used for Synchrony respiratory compensation verification as part of recommended quarterly QA, as well as independent verification of CyberKnife dose calibration and output factor measurements during annual LINAC QA [28]. The experiment involved setting up and irradiating RADPOS inside of a water-equivalent breast phantom, which itself was on top of a dynamic, moving phantom. This experiment is referred to as the Breast Experiment.

2. Can RADPOS be used to evaluate how CyberKnife treatment delivery is affected by complex phantom motion and respiratory hysteresis effects? Combining the RADPOS together with the Quasar respiratory motion phantom and modified lung insert could be an effective approach to evaluating the accuracy of CyberKnife TPS tissue inhomogeneity corrections for the ray-tracing and Monte Carlo dose calculation algorithms, as recommended in TG-135 [28]. An experiment that would closely mimic a real patient treatment on the CyberKnife 
was designed and carried out on a lung-like phantom. Unique experimental aspects included using real patient breathing waveforms, delivering a treatment based on a more complex patient treatment plan and introducing a phase shift between surface motion and tumor phantom motion. This experiment is referred to as the Lung Experiment.

The accuracy of RADPOS dose measurements was evaluated by comparing to film measurements and to CyberKnife TPS calculations. RADPOS was also used to verify internal fiducial and external LED position measurements by comparing to those measured and predicted by the Synchrony motion compensation system. 


\section{Materials and Methods}

This chapter will outline the various systems, processes and apparatus which were instrumental in the design, execution and analysis of the breast and lung experiments. This includes the CyberKnife and RADPOS systems, analysis of radiochromic film and positional data, static and dynamic phantoms, an x-ray imaging dose investigation, and experiment-specific methodology. 


\subsection{General}

\subsubsection{CyberKnife}

\subsubsection{Overview}

Figure 2.1 shows The Ottawa Hospital Cancer Centre's CyberKnife treatment room. On the left is the robotic delivery system, which includes the CyberKnife LINAC, robotic manipulator, and collimation system. There is also a mobile treatment couch, where patients are positioned and translational and rotational setup errors are corrected for. The imaging system consists of the stereoscopic camera system and the $\mathrm{x}$-ray system. The stereoscopic camera detects and tracks the LED optical markers used for external motion tracking. Attached to the ceiling and floor is the x-ray system, which images and tracks the metal fiducial markers or bony anatomy to be used by the tracking system for target localization.

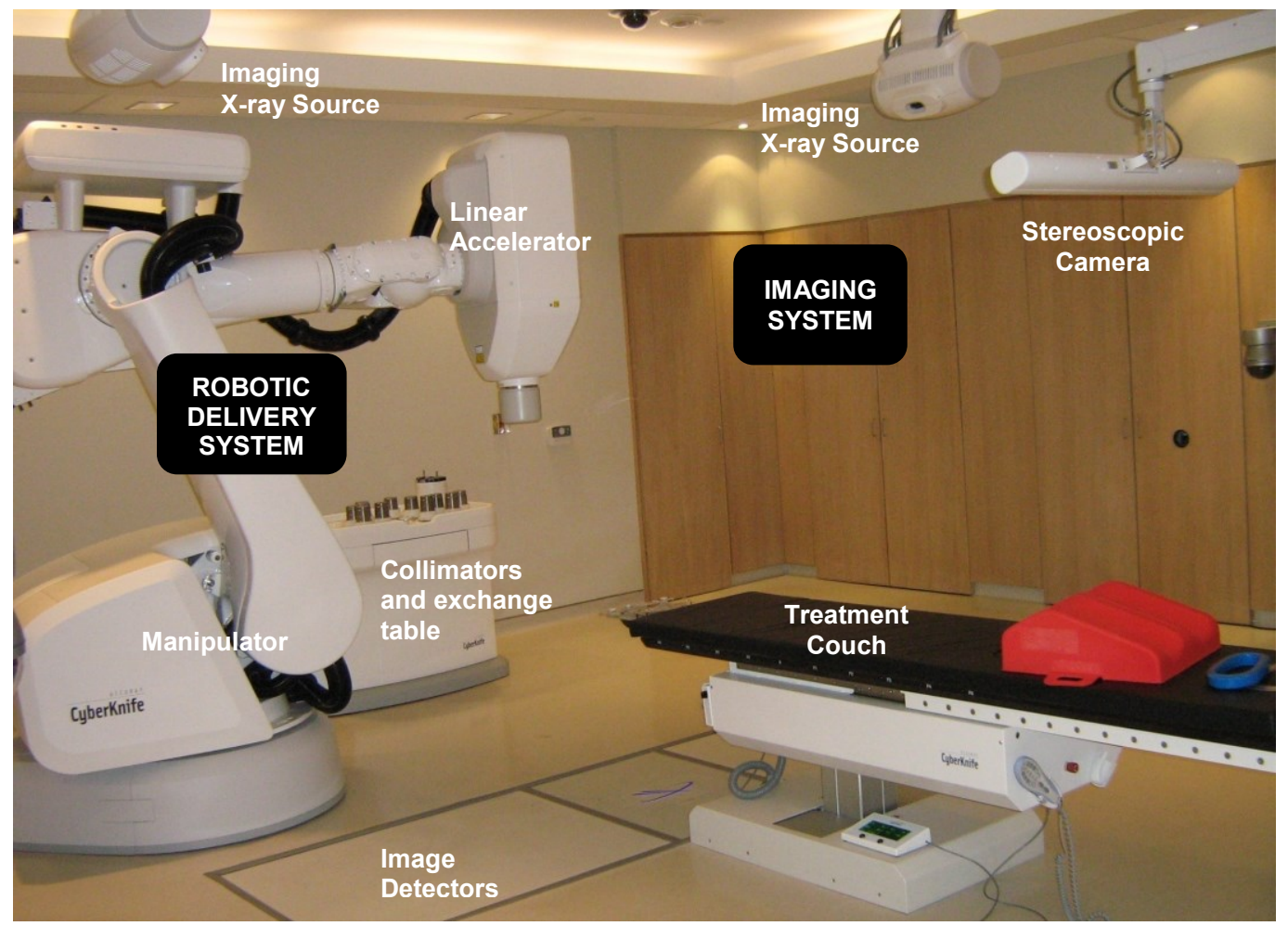

Figure 2.1 CyberKnife treatment room at the Ottawa Hospital Cancer Centre. The robotic delivery system and imaging system are shown, along with their associated components. 


\subsubsection{Robotic Delivery System}

The CyberKnife radiosurgery system consists of a compact linear accelerator mounted on a jointed robotic arm (KUKA Roboter GmbH, Germany), which gives it up to six degrees-of-freedom of motion [29].

The flattening-filter-free LINAC produces $6 \mathrm{MV}$ photon therapy beams at a nominal dose rate of $800 \mathrm{cGy} / \mathrm{min}$ [30]. It is able to deliver small, high dose-rate radiotherapy beams to the tumor from many different non-coplanar directions, resulting in highly conformal dose distributions. The robotic manipulator has been programmed to move the LINAC MV treatment source along 'paths' to various 'nodes' located on a sphere [30], as shown in Figure 2.2. The center of the sphere corresponds to the point at which the orthogonal $\mathrm{kV}$ imaging system beams intersect (referred to as the imaging isocenter). Beam collimation is accomplished via 12 circular tungsten alloy collimators, ranging in size from 5 to $60 \mathrm{~mm}$ in diameter [29]. These collimators can be switched either manually or automatically, allowing the CyberKnife treatment beams to adapt to various tumor shapes and sizes.

CyberKnife is capable of delivering treatments in two ways: isocentric, where the central axes of all the treatment beams share a common intersection point, and nonisocentric, where the treatment beams do not necessarily share a common intersection point. While non-isocentric deliveries may lengthen treatment time, they are the most common type of treatments as they allow the CyberKnife to deliver highly conformal dose distributions to irregularly shaped target geometries. A typical treatment takes between 15-45 minutes, which includes patient preparation, setup, etc. [31]. CyberKnife radiotherapy is generally hypofractionated (i.e., administered over a few fractions). 


\subsubsection{Imaging System and Marker Tracking}

CyberKnife's imaging system consists of two sub-systems: the stereoscopic camera system, which detects and tracks LED optical markers for external motion tracking, and the x-ray imaging system, which images and tracks implanted metal fiducial markers for internal motion tracking. Together, the LED and fiducial position measurements are used by CyberKnife's Synchrony Respiratory Motion Tracking System to generate a predictive model of the target location.

Up to three LED optical markers are positioned on the patient's chest or abdomen for external motion tracking, to be used as a surrogate for the respiratory cycle. A stereoscopic camera, consisting of three CCD cameras, is attached to an adjustable arm and tracks the LED positions at a rate of approximately $25 \mathrm{~Hz}$ (period of $40 \mathrm{~ms}$ ).

In addition, several metal fiducial markers are implanted within or adjacent to the tumor for internal motion tracking. The fiducials are generally made of gold or platinum and are cylindrical in shape, with a diameter of about $1 \mathrm{~mm}$ and a length of 3 to $5 \mathrm{~mm}$. Two orthogonally positioned $\mathrm{x}$-ray sources (peak voltage of $150 \mathrm{kVp}$ ) are fixed to the ceiling, with their corresponding detectors located in the floor [30], as pictured in Figure 2.3. X-ray images are generally taken only once or twice per minute in order to avoid scatter from the LINAC when the beam is on, as well as in order to limit additional dose to the patient. 


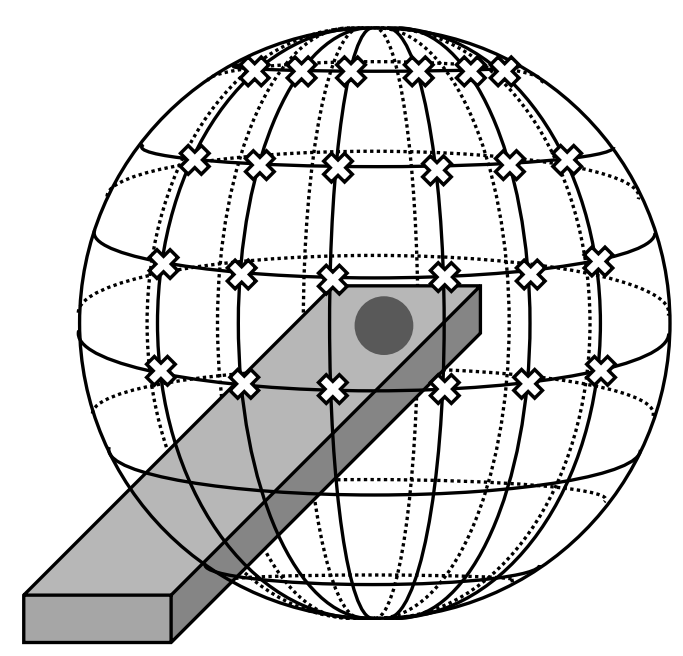

Figure 2.2 Schematic of nodes ( $x$-marks) and paths (connecting lines) surrounding the patient couch of the CyberKnife. The imaging isocenter is represented by the solid circle. Adapted from Dieterich el al., 2011 [28].
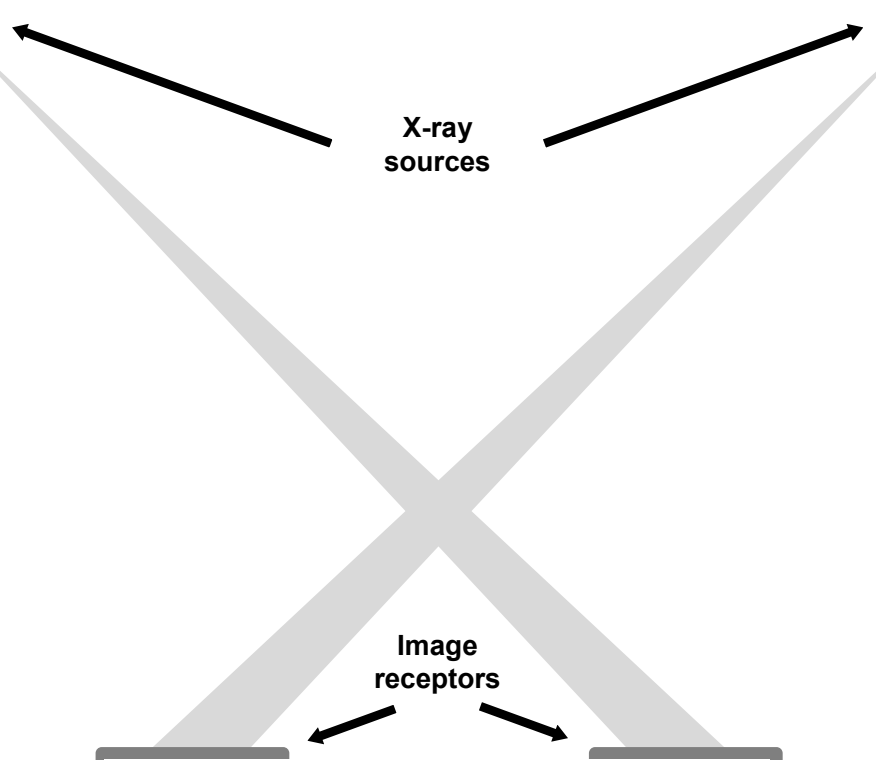

Figure 2.3 Layout of CyberKnife's x-ray imaging system. The $x$-ray sources are mounted to the ceiling orthogonal to one another, and their associated image receptors are located in the floor. Adapted from Dieterich el al., 2011 [28].

\subsubsection{Synchrony Respiratory Motion Tracking System}

The Synchrony Respiratory Motion Tracking System adjusts the direction of the beams during treatment to correct for tumor motion due to breathing. This tracking system employs external optical LED markers and the imaging of internally implanted 
fiducials to build a predictive correlation model, which dynamically corrects beam delivery based on a patient's breathing pattern. Throughout a treatment, the model is continuously updated with the latest tracking data, correcting for drifts in the patient respiratory pattern.

At the start of each treatment, a new correlation model is built from fiducial and LED position measurements to correct for the inter-fraction variability of patient respiratory motion. Multiple x-ray images are taken, in addition to continuous stereoscopic camera tracking, until enough sample points of the patient's respiratory cycle are acquired in order to identify an appropriate model which correlates the external LED motion to the internal fiducial motion. The monitoring of internal target and external surrogate motion continues over the course of a treatment, where the first-in first-out method is used to update the model each time a new x-ray image is taken [32]. This way, intra-fractional changes in a patient's breathing pattern are accounted for, allowing for unrestricted, free breathing of the patient during treatment.

The naturally complicated motion relationship between the chest wall and the lung results in an observable phase difference between measured internal fiducial and external LED position measurements. This effect (hysteresis) leads to a non-linear internal-to-external motion correlation, requiring a linear, quadratic, or constrained fourth order polynomial model fit, depending on the amount of time delay between the measured motion signals [32].

The Synchrony system uses a series of steps to go from marker position measurements to robot beam delivery corrections. First, a separate correlation model is created for every LED optical marker, which matches the movement of that particular 
marker with the motion of the target in order to obtain a target position estimate. Then, the average of these estimates, known as the modeler, gives the final estimate of the target's position. But inherent hardware and software limitations within the CyberKnife system, such as robotic manipulator inertia and latencies in communication, result in an overall delay of approximately $115 \mathrm{~ms}$ between processing of modeler information and adjustment of the LINAC position. In order to compensate for this delay, a predictor is implemented which uses past motion information. Lastly, the predictor output is then fed through a smoothing filter and used to reposition the CyberKnife robot [32].

\subsubsection{Synchrony Log Files}

CyberKnife's Synchrony system generated multiple log files containing Synchrony motion tracking information. Of these log files, only three were used for experimental data analysis: Markers.log, ModelPoints.log and Modeler.log.

The Markers.log file contained information associated with the LED optical markers, which were imaged by the stereoscopic camera. Information relevant to experimental analysis included data point timestamps and individual marker coordinates and statuses (e.g., visible, not visible, etc.)

Information in the ModelPoints.log file corresponded to the data obtained when

the fiducials were imaged by the x-ray sources. Information relevant to experimental analysis included data point timestamps, individual fiducial coordinates and Synchrony correlation errors. These errors represented the differences between where the Synchrony model calculated the fiducials to be and where they actually were (based on position data acquisition via $\mathrm{x}$-ray imaging system). 
The Modeler.log file contained the Synchrony correlation model data, which was computed based on LED and fiducial position measurements. This data included model data point timestamps and calculated target coordinates.

\subsubsection{RADPOS}

\subsubsection{Overview}

RADPOS is a 4D dosimetry system consisting of a probe which combines a highsensitivity microMOSFET dosimeter (TN1002RDM, active volume: $0.2 \times 0.2 \times 1 \times 10^{-3}$ $\mathrm{mm}^{3}$ ) and an electromagnetic positioning sensor. RADPOS has the ability to perform real-time dose and position measurements simultaneously, making it an excellent candidate for acting as an independent quality assurance tool for the CyberKnife system. Figure 2.4a shows what the RADPOS system looks like. The microMOSFET reader is responsible for dose measurements, and the transmitter, pre-amplifier and 3D-guidance tracker are responsible for position measurements.

The position sensor and microMOSFET are separated by $8 \mathrm{~mm}$, as seen in Figure $2.4 \mathrm{~b}$, in order to limit radiation attenuation and disturbance of the particle fluence near the dosimeter. In order to perform dose measurements, RADPOS' microMOSFET dosimeter measures a threshold voltage difference which is induced when the microMOSFET is irradiated. It is this threshold voltage difference that is proportional to the absorbed dose. In terms of position measurements, the DC magnetic field transmitter emits a pulsed 3D magnetic field which is detected and used by RADPOS' EM positioning sensor in order to determine its position coordinates. 
Logging of the dose can occur either manually or automatically at intervals defined by the user. Similarly, logging of the position can occur either manually or automatically, up to a frequency of about $20 \mathrm{~Hz}(50 \mathrm{~ms})$. RADPOS is capable of logging up to 10000 position data entries, at which point logging will stop and the data must be saved and cleared for subsequent use. Logging at max rate (i.e., $20 \mathrm{~Hz}$ ) allows for only $500 \mathrm{~s}(8.3 \mathrm{~min})$ of continuous data collection, and so a logging rate of $10 \mathrm{~Hz}(100 \mathrm{~ms})$ was used during experiments as a compromise between temporal resolution and longer data collection time (1000 s, or $16.7 \mathrm{~min})$.
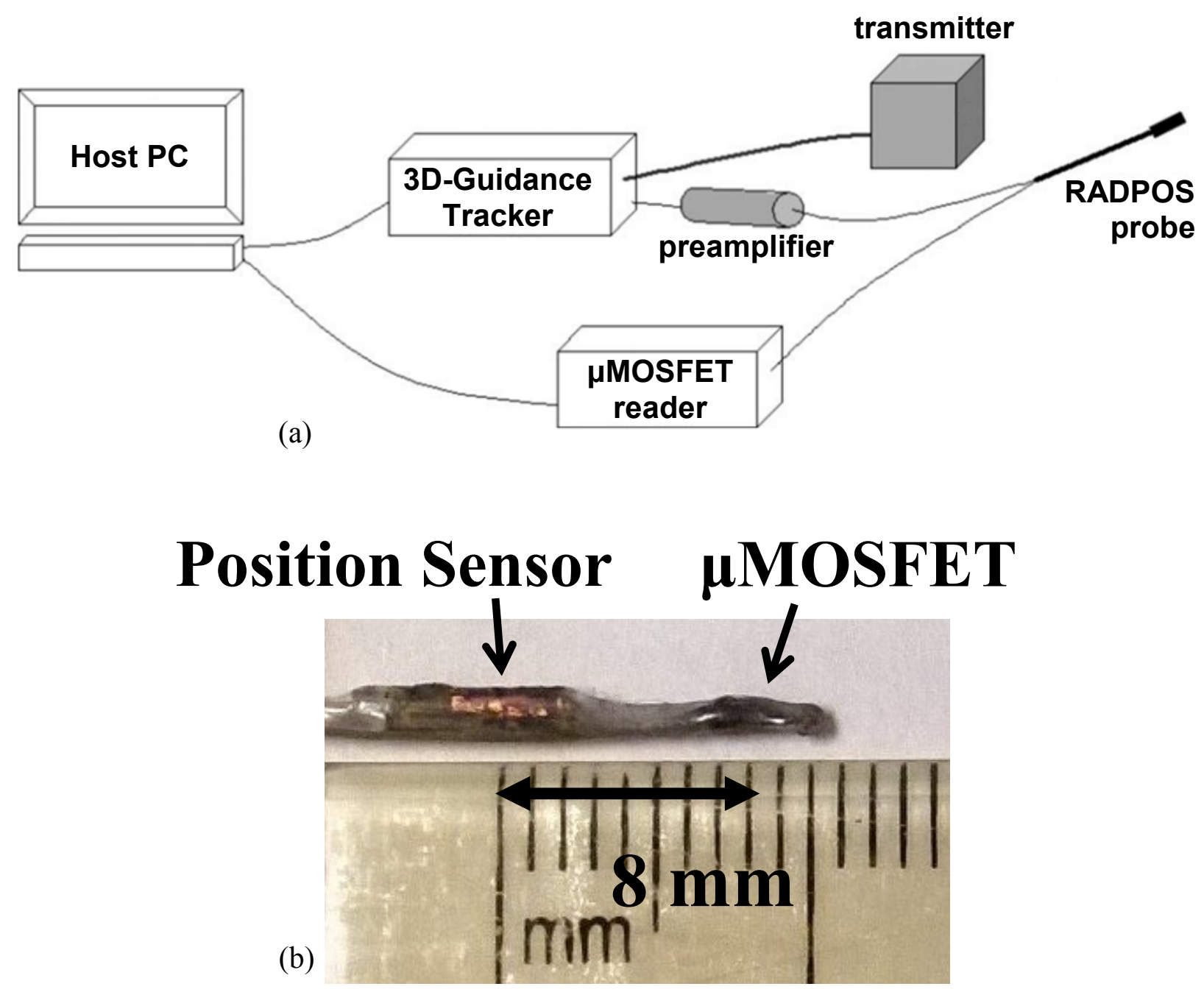

Figure 2.4 RADPOS 4D dosimetry system. An overview of RADPOS' components, adapted from Cherpak el al., 2009 [30], is displayed in (a), while a closer view of the dosimeter probe is shown in (b). 


\subsubsection{Dose Measurement and Calibration}

The RADPOS system uses a metal-oxide-semiconductor-field-effect transistor, or MOSFET, dosimeter in order to measure absorbed dose. Figure 2.5 shows how the MOSFET is structured. The basic working principle of the MOSFET is that the conductivity of a source-to-drain channel is controlled by a voltage which is applied to the gate.

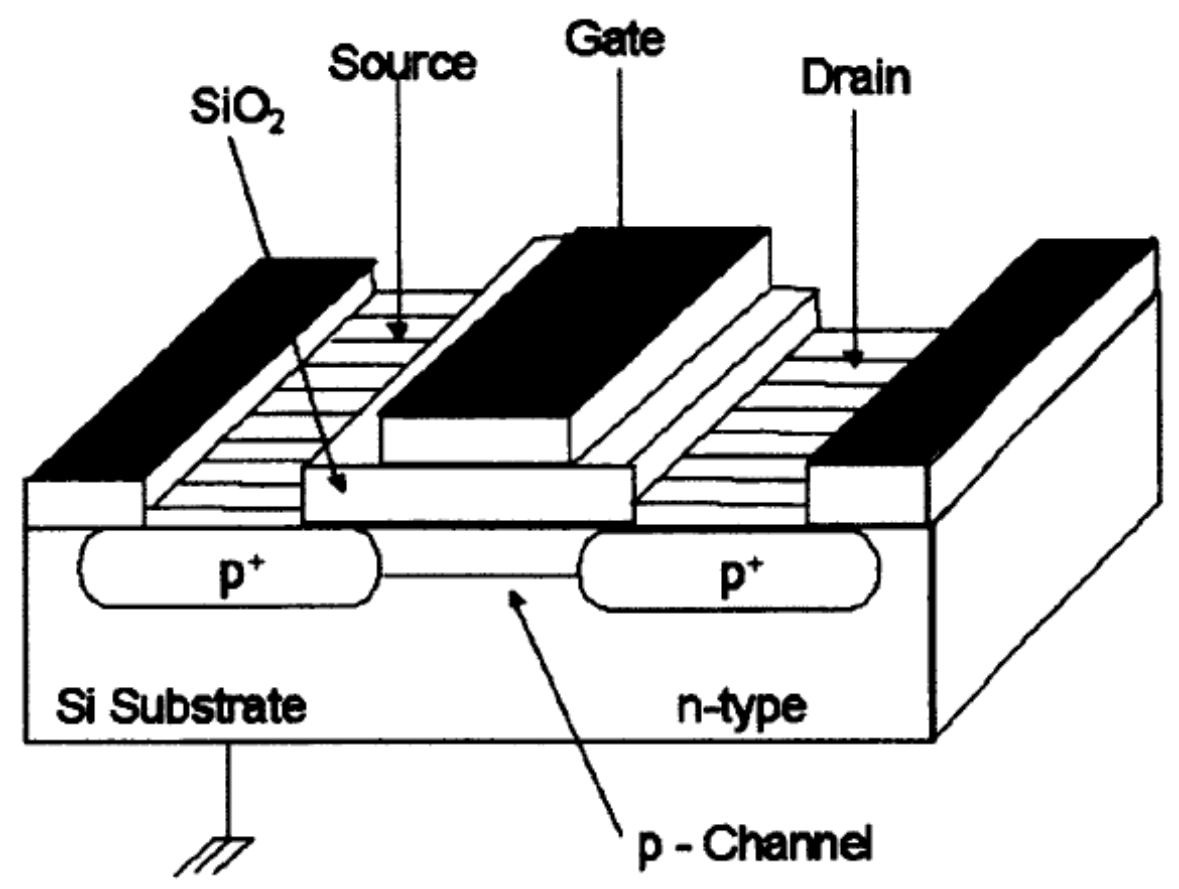

Figure 2.5 MOSFET dosimeter, which is part of the RADPOS probe. Adapted from Soubra et al., $1994[25]$.

During normal (i.e., non-irradiated) MOSFET reading, a negative gate voltage, $\mathrm{V}_{\mathrm{g}}$ $<0$, is applied. As a result, holes are attracted towards the channel from the negatively doped Si substrate. This causes the channel to become more positive and conducting. At a certain threshold voltage, $\mathrm{V}_{\text {th }}$, the channel is conductive enough (i.e., enough positive charge build-up) such that the source, gate, and drain become connected, creating an inversion layer [27]. 
During exposure to ionizing radiation, a positive bias is applied to the MOSFET gate. Irradiation produces electron-hole pairs in the $\mathrm{SiO}_{2}$ layer, which lies right beneath the gate. Some holes recombine with electrons, while others are repelled from the positive gate towards the oxide-substrate interface and become trapped. When the MOSFET is read using an applied negative $\mathrm{V}_{\mathrm{g}}$, the extra positive charge at the interface (due to the trapped holes) repels and impedes substrate hole build-up. This causes a shift in the gate voltage necessary to produce an inversion layer, where a higher applied voltage is required and $\mathrm{V}_{\text {th }}$ has to be increased to overcome the positive potential barrier, as shown in Figure 2.6. This shift in the threshold voltage, $\Delta \mathrm{V}_{\text {th }}$, is directly proportional to the radiation dose, enabling the RADPOS to perform dose measurements [27].

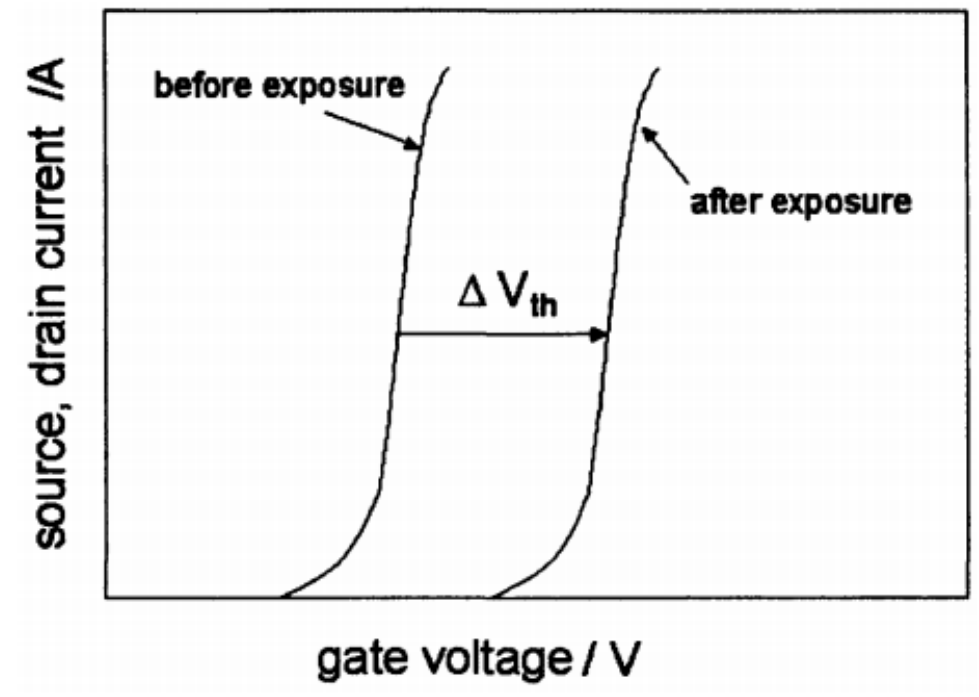

Figure 2.6 Change in the MOSFET's threshold voltage due to ionizing radiation. Adapted from Soubra et al., 1994 [25].

When the MOSFET is irradiated, newly formed holes that did not recombine with electrons will eventually become trapped in the oxide-substrate interface. This is what causes a change in the measured threshold voltage (which is proportional to the absorbed dose). These trapped holes persist for a very long time (i.e., years), and every time the 
MOSFET is irradiated, more and more holes become trapped. A larger number of trapped holes make it more and more difficult for other holes to approach the interface due to the positive charge build-up. This causes an overall decrease in MOSFET sensitivity, and therefore, a change in the calibration coefficient, CF.

\subsubsection{RADPOS Cross-Calibration}

The CF, given in $\mathrm{cGy} / \mathrm{mV}$, was calculated for a RADPOS dosimeter by taking the quotient of the delivered dose (cGy) and the change in the MOSFET's threshold voltage $(\mathrm{mV})$, as given in Equation 2.1:

$$
C F=\frac{D}{\Delta V_{t h}}
$$

RADPOS threshold voltage measurements were made before and after irradiation with a known dose, and this was repeated several times. Then, the average threshold voltage difference was used to determine the CF [34].

\subsubsection{Position Measurement}

The RADPOS system uses an electromagnetic positioning sensor and DC magnetic field transmitter in order to perform position measurements. The positional coordinates are determined by the sensor's response to the pulsed, 3D magnetic field generated by the DC magnetic field transmitter.

A current is sequentially applied to three perpendicularly-arranged coil loops within the transmitter, producing well-characterized magnetic fields along the $\mathrm{x}, \mathrm{y}, \mathrm{z}$ directions. The field in each direction has a trapezoidal amplitude profile, as pictured in Figure 2.7. The purpose of the steady-state period is to give ample time for eddy currents 
induced in nearby metals to decay and for the output of the position sensor to stabilize [34].

The position sensor consists of a three-axis ring fluxgate magnetometer and is responsible for measuring the strength of the generated magnetic field, which is proportional to the inverse cube of the distance from the transmitter [35]. The sensor's positional results are converted into $\mathrm{x}, \mathrm{y}, \mathrm{z}$ coordinates, as well as azimuth, elevation, and roll angles. The magnetic field transmitter, as well as its coordinate system, is shown in Figure 2.8.

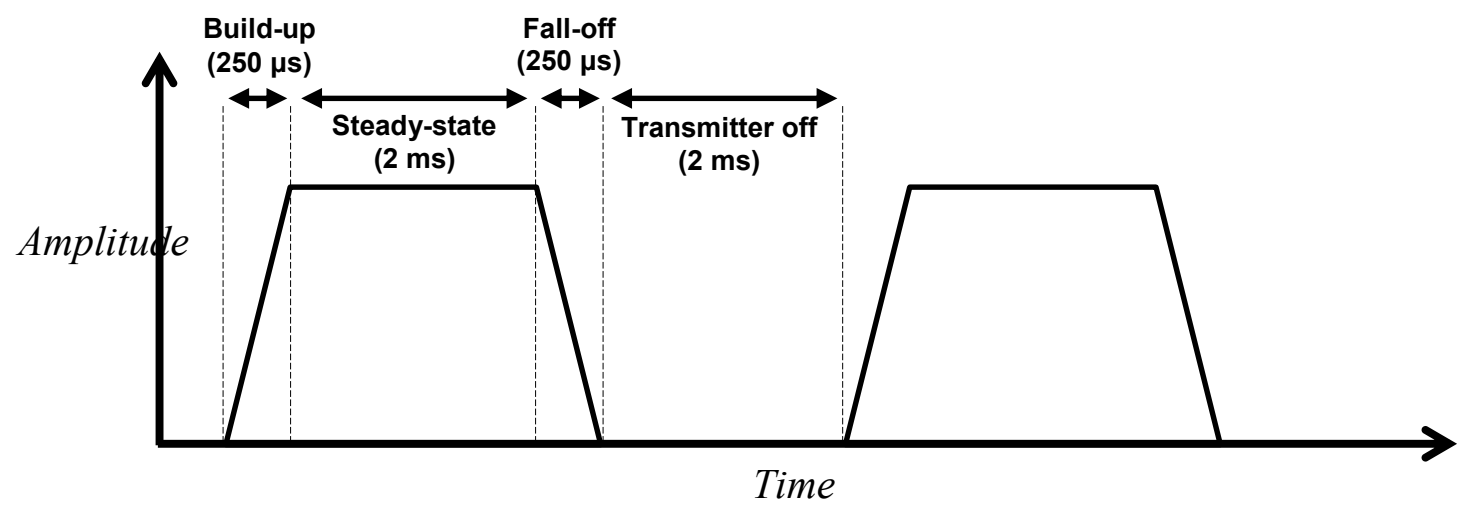

Figure 2.7 The amplitude profile of the magnetic field produced by the DC magnetic field transmitter has a trapezoidal shape.

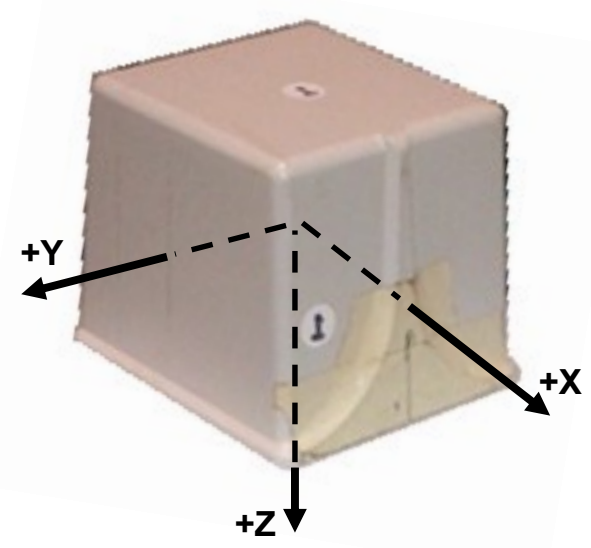

Figure 2.8 RADPOS' DC magnetic field transmitter. Also shown is its coordinate system. 


\subsubsection{Film}

\subsubsection{Overview}

EBT3 GafChromic film (Ashland Inc.) was used to perform dose measurements, which were compared to RADPOS dose measurements and treatment planning system dose calculations.

This film consists of a $27 \mu \mathrm{m}$ thick active substrate layer sandwiched between two $120 \mu \mathrm{m}$ thick clear polyester layers [36]. The active layer is composed of lithium salt monomer crystals dispersed in gelatin. Upon irradiation, the monomers polymerize, darkening the film and effectively increasing its optical density [37]. The measured change in optical density between pre- and post-irradiation is then proportional to the absorbed dose.

Preparation of the film for use in an experiment involved cutting it into specific sizes and performing pre-irradiation scans using a large Epson 10000XL scanner (SN: FVS0016178). Scan settings were set to those shown in Figure 2.9a, and in addition to the adjustments shown in Figure 2.9b, no color correction was applied. A custom acrylic sheet template was used in order to improve film placement stability and scan reproducibility. A post-irradiation scan was then done a few days after the experiment in order to minimize uncertainties associated with the time dependence after exposure for GafChromic film [38]. 


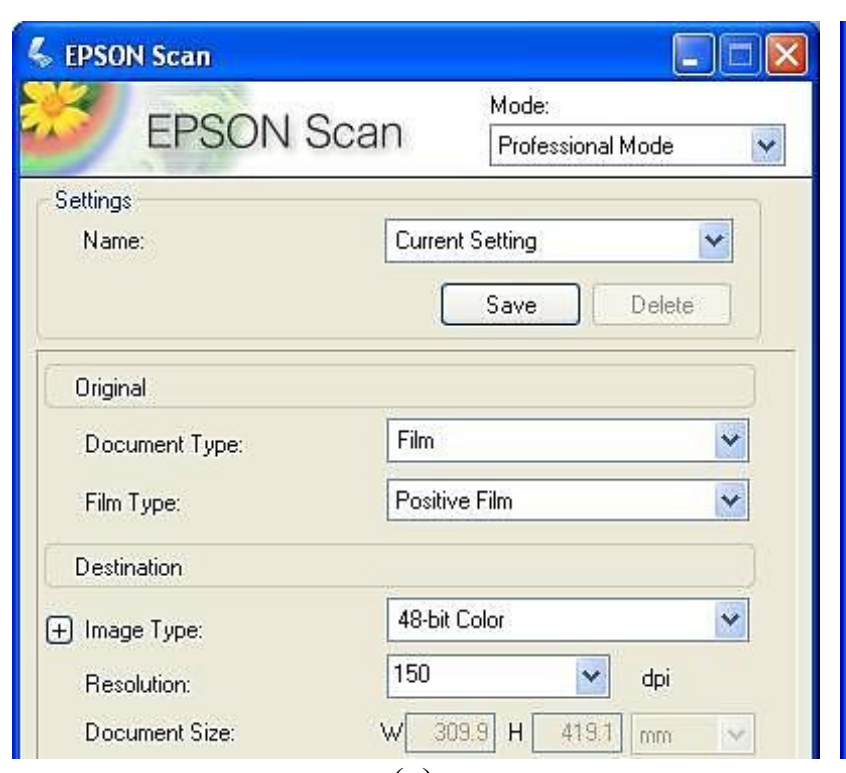

(a)

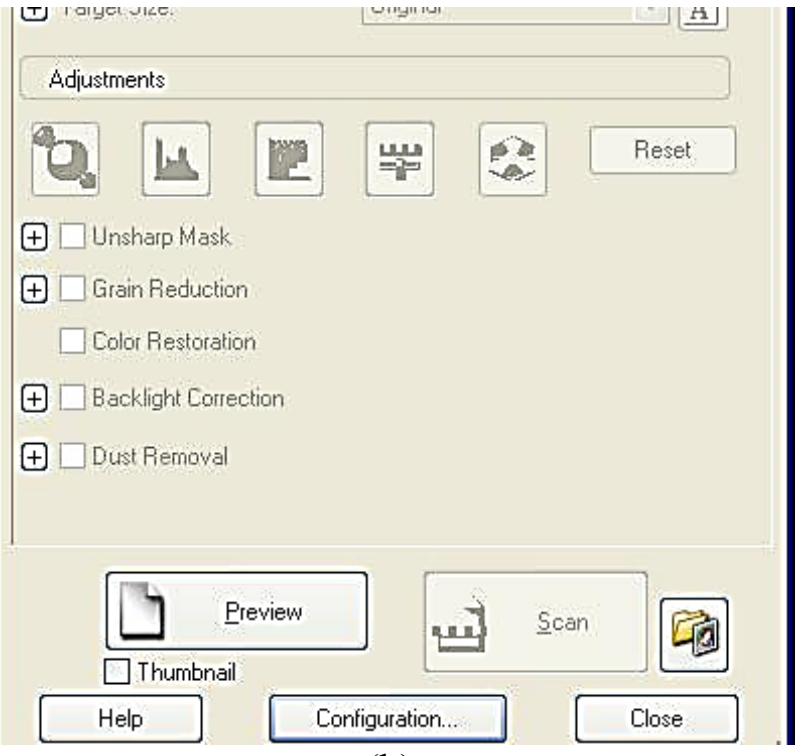

(b)

Figure 2.9 Scan settings (a) and adjustments (b) used for EBT3 GafChromic film processing.

\subsubsection{Analysis}

Once all pre- and post-irradiation scans were completed, an in-house MATLAB script was used to analyze the films and retrieve the dosimetric results. The script has the user import CT scan images and the treatment planning system calculated dose matrix. Then, the user identifies the film plane and fiducials on the CT, followed by the generation of dose film files. These files take into account dose rescaling, as well as color channel uniformity corrections. Next, the fiducial locations are identified on the film files, and the film and CT are aligned (based on matching the fiducial positions).

A gamma analysis can then be performed and a region-of-interest, ROI, is chosen. This should correspond to the region of the film nearest to the detector (i.e., RADPOS dosimeter probe). Finally, a dose value (mean, min, and max) is generated for that film's selected ROI. 
A dose rescaling was performed using a similar approach to that used by Lewis $e t$ al. [39]. The film piece which was left unirradiated between pre- and post-irradiation scans was used to account for changes in the apparent darkness of the film not due to radiation exposure (e.g., thermal and ambient light conditions, scanner lamp brightness variation, etc.). The post-irradiation film intensity values were adjusted to give zero average dose to a large rectangular region of interest in the unirradiated film. Hence, the other film data which were scanned at the same time as the unirradiated film were scaled using the same scaling factor. In a similar manner, the film piece which was irradiated to a known reference dose was used for known dose rescaling. However, the known dose film was rescaled after applying the film's calibration parameters.

A gamma analysis is performed based on the choice of dose-difference and distance-to-agreement parameters, and is used to compare measured (e.g., film) and calculated (e.g., TPS) dose distributions [40]. Mathematically, a gamma analysis is computed according to Equation 2.2:

$$
\gamma=\min \left\{\sqrt{\left(\frac{r}{\Delta d}\right)^{2}+\left(\frac{\delta}{\Delta D}\right)^{2}}\right\}
$$

where $r$ is the spatial difference between a measurement point and a point on the calculated dose distribution, $\Delta d$ is the distance-to-agreement criterion, $\delta$ is the difference between the calculated and measured dose values at each point, and $\Delta D$ is the dosedifference criterion. Minimization is done over a small region around each calculated data point for the entire dose distribution (i.e., entire film). 


\subsubsection{Processing}

Figure 2.10 gives examples of film scans before (Figure 2.10a) and after (Figure 2.10b) experimental irradiations have been done, along with the various film sizes used. Also shown around the edges of each film scan is the custom acrylic sheet template, used to improve film placement stability and scan reproducibility.

A $7.62 \times 12.7 \mathrm{~cm}^{2}$ piece of film was irradiated during the experimental CyberKnife treatment (left film in Figure 2.10b). A second $7.62 \times 12.7 \mathrm{~cm}^{2}$ piece of film irradiated to a dose of 300 cGy was used for known dose rescaling (middle film in Figure 2.10b). The final $5.08 \times 12.7 \mathrm{~cm}^{2}$ piece of film was left unirradiated and was used for zero dose rescaling (right film in Figure 2.10b).

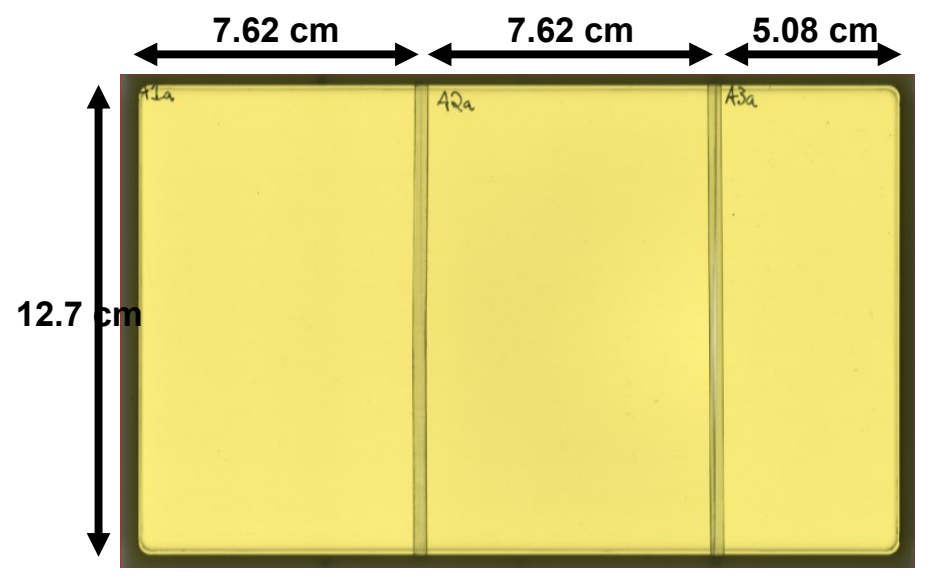

(a)

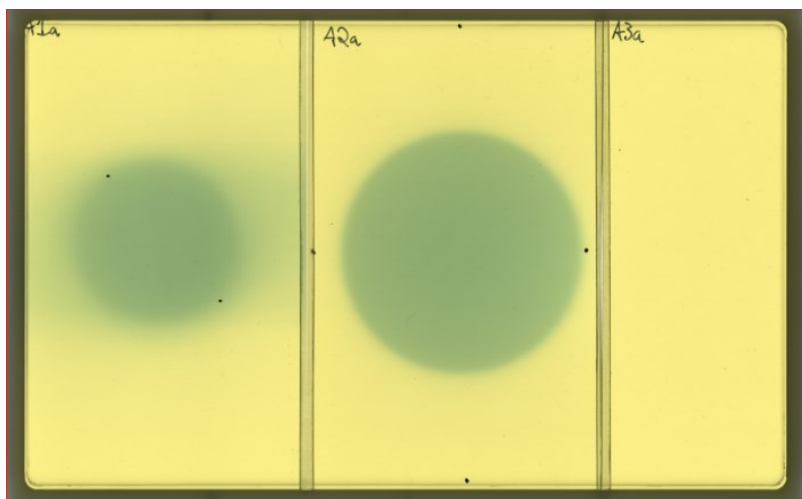

(b)

Figure 2.10 Film arrangements and sizes used for pre- and post-experiment analysis. Shown are the films before (a) and after (b) irradiation, along with the sizes of each film piece. 


\subsubsection{Calibration}

A series of $5.08 \times 12.7 \mathrm{~cm}^{2}$ pieces of film were irradiated one at a time to doses of 10 to 300 cGy in a pseudo-random fashion: $10,250,75,200,50,300,150,25$, and 100 cGy. Figure 2.11 shows what the calibration film scans looked like before (Figure 2.11a) and after (Figure 2.11b) irradiation for doses of 75, 100, and $150 \mathrm{cGy}$ (left, mid-left, and mid right films, respectively, in Figure 2.11a,b). A piece of film was left unirradiated and was used for zero dose rescaling (right-most film in Figure 2.11a,b).

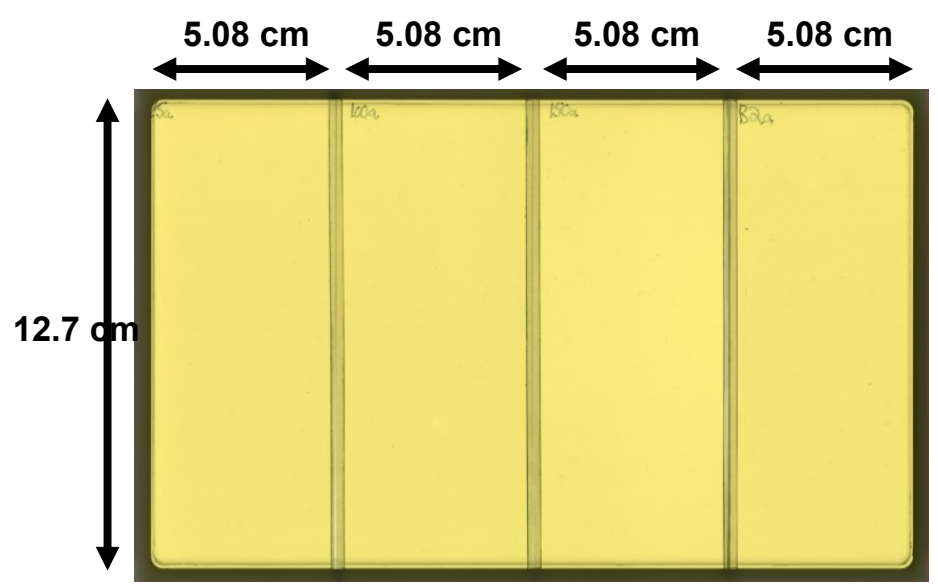

(a)

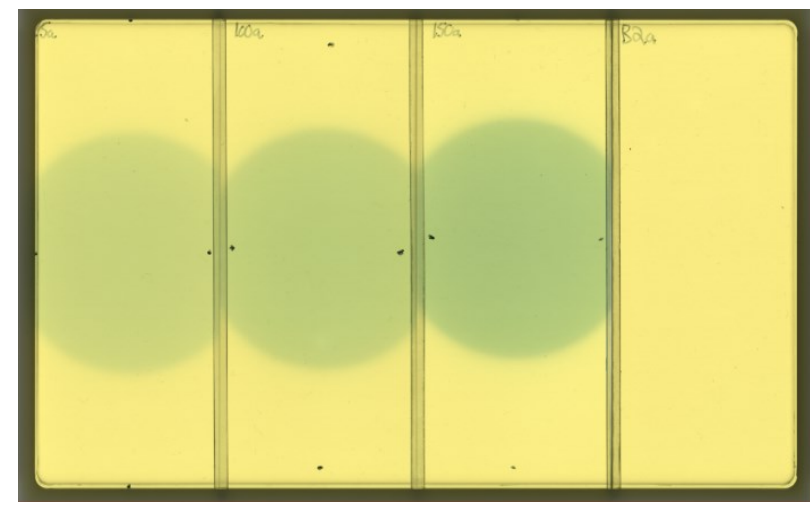

(b)

Figure 2.11 Film arrangements and sizes used for pre- and post-experiment calibration. Shown are the films (a) before and (b) after irradiation, along with the sizes of each film piece.

In order to produce a film calibration curve, the net optical density, OD, and the standard deviation of the net OD values were calculated from the processed calibration film dose values. Based on the dose response relations proposed by Devic et al. [41], the net $\mathrm{OD}$ and the standard deviation of the net OD were calculated according to Equations 2.3 and 2.4 , respectively:

$$
\begin{gathered}
\operatorname{netOD}(D)=\log _{10} \frac{I_{\text {unirr }}(D)}{I_{\text {irr }}(D)} \\
\sigma_{\text {netOD }}(D)=\frac{1}{\ln 10} \sqrt{\left(\frac{\sigma_{I_{\text {unirr }}}(D)}{I_{\text {unirr }}(D)}\right)^{2}+\left(\frac{\sigma_{I_{\text {irr }}}(D)}{I_{\text {irr }}(D)}\right)^{2}}
\end{gathered}
$$


where $I_{\text {unirr }}$ and $I_{\text {irr }}$ are the unirradiated and irradiated intensities, respectively, while $\sigma_{\text {unirr }}$ and $\sigma_{\text {irr }}$ are the unirradiated and irradiated intensity uncertainties, respectively. A net OD versus dose film calibration curve is then created from the computed data. An in-house MATLAB script uses a least-squares-based approach to determine the calibration curve fit parameters ( $a$ and $b$ ) according to Equation 2.5:

$$
f(O D)=a \cdot O D+b \cdot O D^{c}
$$

The c parameter is varied from 1 to 3 in steps of 0.1 , and the value which minimizes an error function (the sum of the square of the residual error) is selected (Figure 2.12).

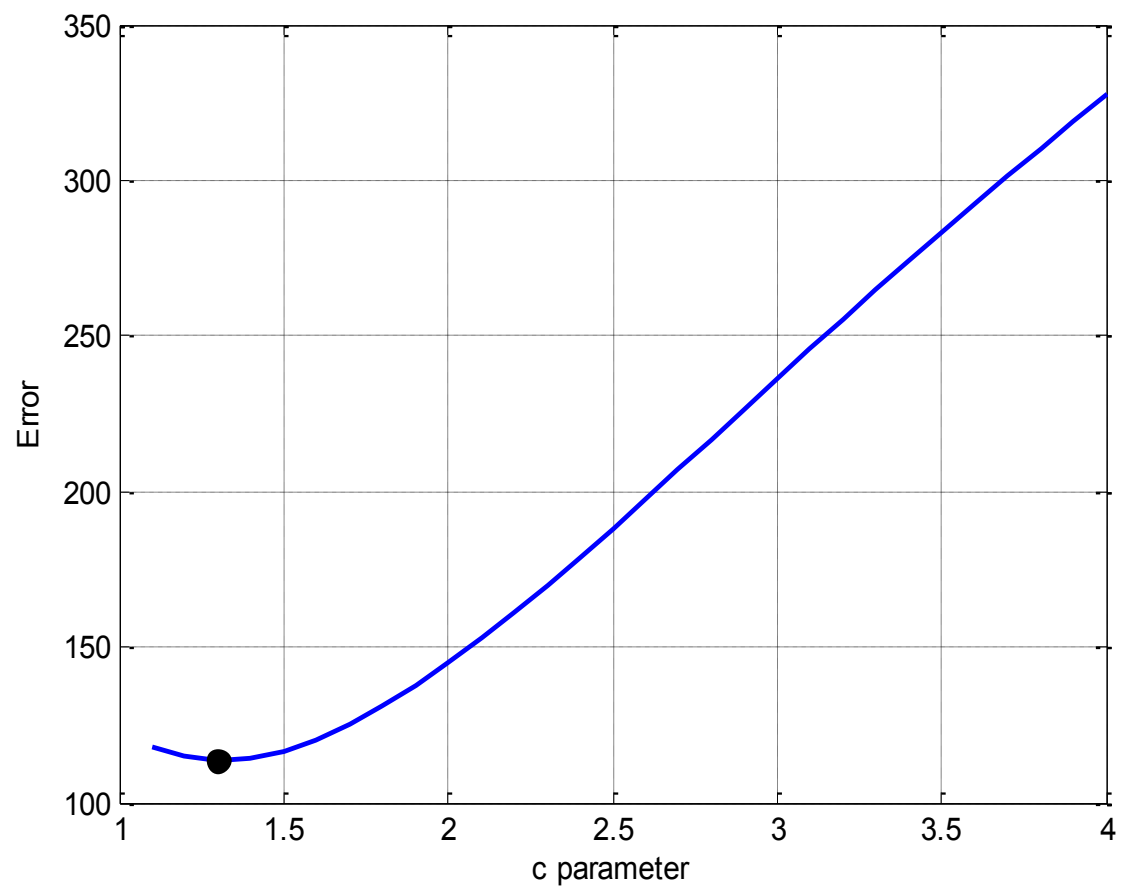

Figure 2.12 Example of a how the c parameter (i.e., calibration curve exponent) is determined. The value of $c$ which minimizes the error function is used for the calibration fit. In this case, a value of $c=1.3$ optimizes the associated netOD versus dose calibration curve fit. 


\subsubsection{Positional Data Analysis}

\subsubsection{Overview}

During an experiment, three position measurement datasets were generated: fiducial $\log$ files for fiducial position measurements, Synchrony log files for LED position measurements, and RADPOS output files for RADPOS position measurements. Each of these position measurement datasets measures motion at a different point in a phantom (which is assumed to move as a rigid body) and each system has a different coordinate system, which prevented dataset comparisons from being done directly. The stereoscopic camera can also be moved around in the room freely changing the origin and orientation of the axes of the LED coordinate system from experiment to experiment. To be able to consistently compare RADPOS position measurements to those of the fiducials and LEDs, a coordinate alignment algorithm is required to compare the three position tracking modalities.

Additionally, data acquisition happened on different computers for different systems: Synchrony and fiducial log files were created on the CyberKnife computer terminal, while RADPOS output files were generated on a separate laptop computer. Therefore, in addition to being in different spatial coordinate systems, the CyberKnife and RADPOS file data were based on separate clocks with different origins in the time domain. It was necessary to synchronize the position measurements both spatially and temporally in order to consistently compare the datasets.

During an experiment, position data logging for the Synchrony and RADPOS systems was initiated several seconds prior to turning on the Quasar in order to produce a clearly visible 'no-motion to motion-on' discontinuity, as seen in Figure 2.13. By finding 
this point in the Synchrony and RADPOS position signals, the difference between timescales could be determined and temporal synchronization could be achieved.

Before any position measurement data analysis could be done, the necessary information was first read in from the fiducial and Synchrony log files, as well as from RADPOS output files. An in-house MATLAB script was written, which enabled the user to select which dataset to import and automatically read in the appropriate information. Depending on the data format, which was slightly different for each position measurement dataset, a number of sorting and filtering steps had to be implemented. For example, the Markers log file, which contained the $\mathrm{x}, \mathrm{y}, \mathrm{z}$ position information for the three LED markers, had the following data format:

$$
\text { [t] (dt) [map]:x1 y1 z1 st1:x2 y2 z2 st2:x3 y3 z3 st3 }
$$

In this case, only the $\mathrm{t}$ and $\mathrm{x}, \mathrm{y}, \mathrm{z}$ information (bolded) was relevant. However, st gave information regarding a certain marker's status (visible, not visible, etc.), and so any instances where all three LEDs did not have a positive status had to be discarded. 

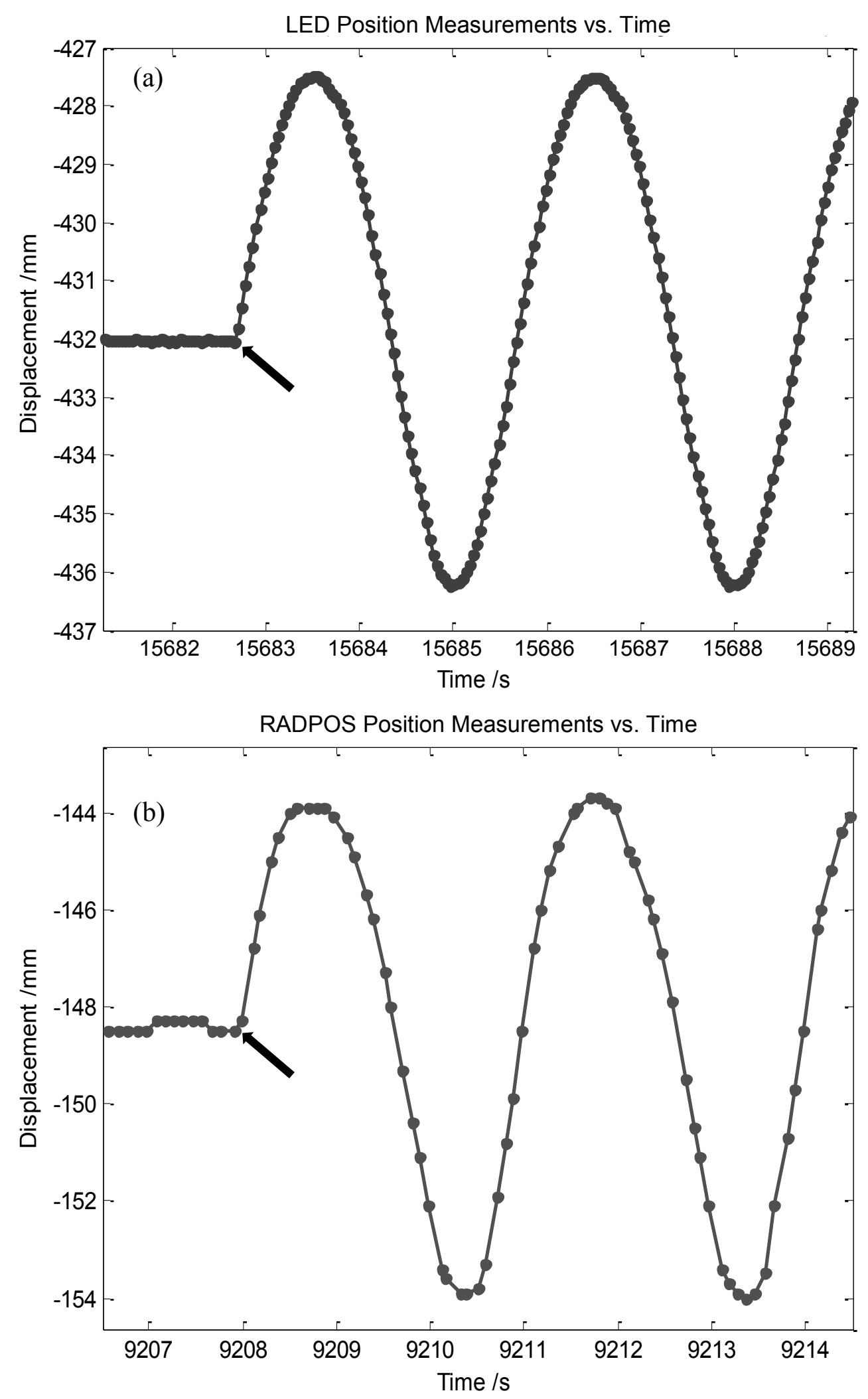

Figure 2.13 Shown are the (a) LED and (b) RADPOS motion signal waveforms. A common feature from both plots, such as the "jump" indicated by the black arrow, was used to temporally align the waveforms (which appear at a different time due to differences in how the temporal origin is defined in the two systems). 


\subsubsection{Coordinate Alignment Algorithm}

An algorithm put forth by Arun et al. [42] and later modified by Umeyama [43] offers a solution to the coordinate alignment problem. A coordinate transformation can be represented as follows:

$$
p_{i}^{\prime}=R \cdot p_{i}+T
$$

where $\mathbf{p}$ and $\mathbf{p}^{\prime}$ are initial and final 3D point sets, respectively, $\mathbf{R}$ is a rotation matrix, and $\mathbf{T}$ is a translation matrix. This algorithm finds $\mathbf{R}$ and $\mathbf{T}$ in order to compute the leastsquares solution to Equation 2.7:

$$
\Sigma^{2}=\sum_{i=1}^{N}\left\|\boldsymbol{p}_{\boldsymbol{i}}^{\prime}-\left(\boldsymbol{R} \cdot \boldsymbol{p}_{\boldsymbol{i}}+\boldsymbol{T}\right)\right\|^{2}
$$

where $\Sigma^{2}$ is the parameter to be minimized and $\mathrm{N}$ is the number of data points. In other words, this least-squares-based coordinate alignment algorithm transforms $\mathbf{p}$ into $\mathbf{p}^{\prime}$ via unique rotation and translation matrices. In the context of this work, the LED and RADPOS position measurements were transformed into the coordinate system of the fiducials, which is considered to be the absolute coordinate system of the room. The three position tracking modalities could then be compared consistently within a common coordinate system.

The coordinate alignment algorithm was applied for position measurement comparison during analysis of the breast and lung experiments. In addition, the algorithm allowed for position data comparisons along the $\mathrm{x}, \mathrm{y}, \mathrm{z}$ directions of the position tracking modalities.

The algorithm could only be used if $\mathbf{p}$ and $\mathbf{p}^{\prime}$ were datasets of the same size. Since the various position measurement modalities sampled motion at different frequencies and 
output differently-sized datasets, the algorithm was implemented as follows: Let L be the number of LED data points (very finely sampled), A be the number of RADPOS data points (finely sampled), and F be the number of fiducial data points (sparsely sampled) for a particular position measurement.

1. The LED data was organized into a $3 \times \mathrm{L}$ matrix (row 1 corresponded to $\mathrm{x}$ measurements, row 2 to $y$, row 3 to $z$ ), the RADPOS data into a $3 \times A$ matrix, and the fiducial data into a $3 \times \mathrm{F}$ matrix

2. The LED matrix was interpolated to the fiducial time data points, resulting in a $3 \times \mathrm{F}$ matrix with LED data (which will be referred to as $3 \times \mathrm{L}_{\mathrm{F}}$ )

3. The coordinate alignment algorithm was run to obtain the rotation $(3 \times 3$ matrix $\mathrm{R})$ and translation $(3 \times 1$ vector $\mathrm{T})$ matrices that transformed $3 \times \mathrm{L}_{\mathrm{F}}$ into the fiducial reference frame, resulting in a $3 \times \mathrm{L}_{\mathrm{F}}^{\prime}$ matrix

4. Steps 2 and 3 were repeated for the $3 \times$ A RADPOS data matrix

5. The transformed LED and RADPOS data, $3 \times \mathrm{L}_{\mathrm{F}}^{\prime}$ and $3 \times \mathrm{A}^{\prime} \mathrm{F}$, were compared within the fiducial reference frame

6. The $\mathrm{T}$ vector was concatenated to itself $\mathrm{L}$ times in order to obtain a $3 \times \mathrm{L}$ translation matrix, $T_{L}$, to be applied to every point in the LED dataset

7. $R$ and $T_{L}$ were applied to the original $3 \times L$ LED data to transform it into the fiducial reference frame, resulting in a $3 \times L^{\prime}$ matrix

8. Steps 6 and 7 were repeated for the $3 \times$ A RADPOS data matrix

9. The transformed LED and RADPOS data, $3 \times \mathrm{L}^{\prime}$ and $3 \times \mathrm{A}^{\prime}$, were compared within the fiducial reference frame (which involved interpolating the very finely sampled transformed LED matrix to the finely sampled RADPOS time data points) 
All interpolations were done using cubic spline interpolation. Comparison of RADPOS to the Synchrony predictive correlation model was accomplished in exactly the same manner as outlined above, where the model data (very fine resolution) took the place of the LED data. In subsequent chapters, the comparisons from steps 5 and 9 will be referred to as Comparison 1 and Comparison 2, respectively. 


\subsubsection{Phantoms}

\subsubsection{Breast}

The breast phantom used was made from Certified Therapy Grade (CTG) Solid Water (Gammex Inc.). It was approximately semi-spherical in shape, measuring 11.75 $\mathrm{cm}$ in diameter and $7.94 \mathrm{~cm}$ in height. The phantom was split in half to allow for the insertion of film, and several small slots were drilled in the inner surface in order to house four metal fiducial markers and the RADPOS detector probe (see Figure 2.14).
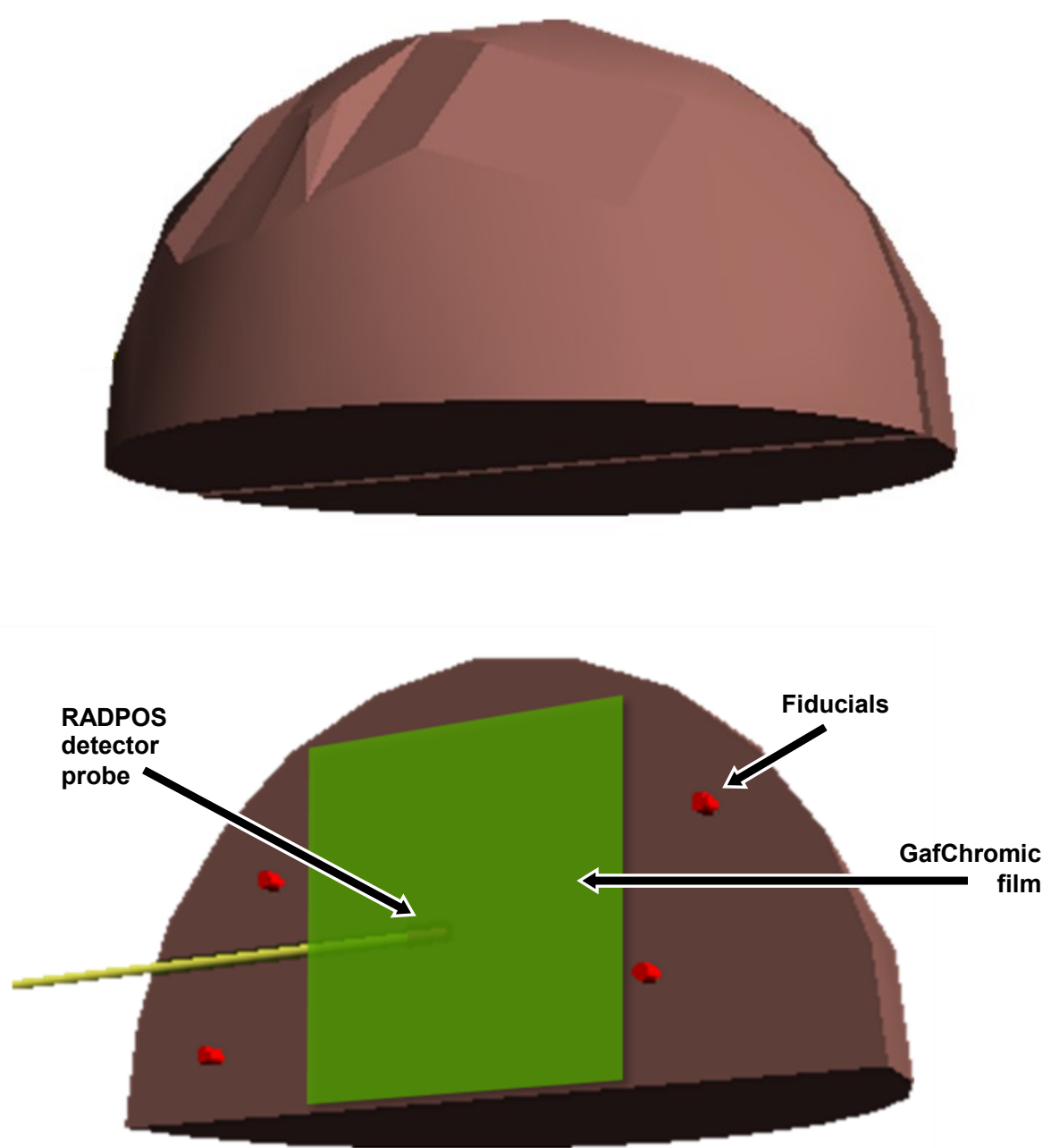

Figure 2.14 3D renderings of semi-spherical Solid Water breast phantom. Both halves (a) and one half (b) of the phantom is shown, along with approximate positions of fiducials and detectors. 


\subsubsection{Lung}

The lung insert (Modus Medical Devices Inc.) used was made from cedar wood. It was cylindrical in shape, measuring $7.90 \mathrm{~cm}$ in diameter and $16.10 \mathrm{~cm}$ in length, with approximately $1 \mathrm{~cm}$ of plastic on either end. The phantom was split into two halves to allow for the insertion of film, and several small slots were drilled in the inner surface in order to house four metal fiducial markers and the RADPOS detector probe. In addition, a spherical groove was cut out of the centre of the insert and filled with spherical CTG Solid Water, meant to represent a solid lung tumor (see Figure 2.15).
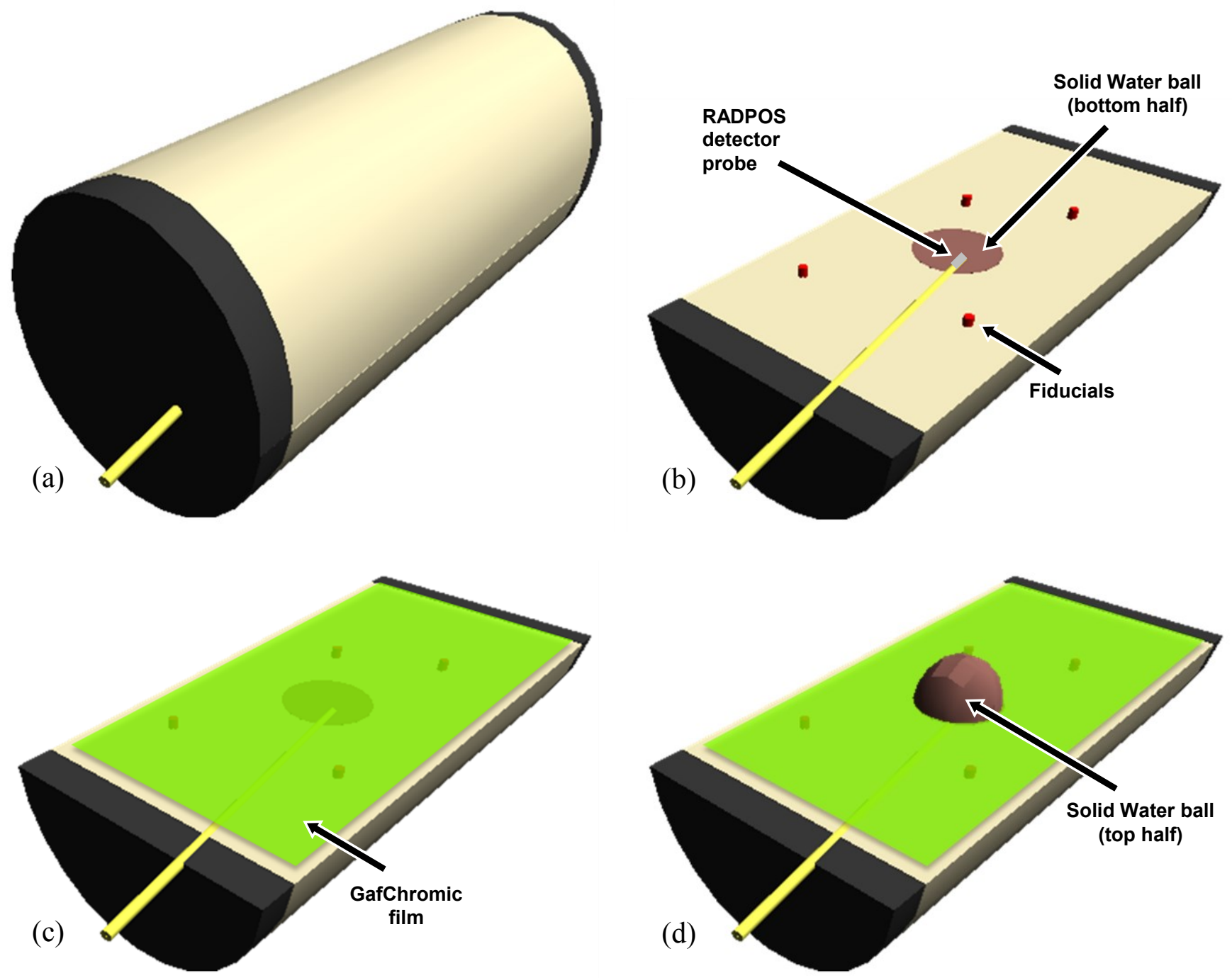

Figure 2.15 3D renderings of cylindrical cedar wood lung insert. Both halves (a) and one half (b,c,d) of the phantom is shown, along with other features common to all experiments. 


\subsubsection{Quasar}

The Quasar Respiratory Motion Phantom (model 100-1011, Modus Medical Devices Inc.) was used to simulate the motion of the chest and lung using the motion of the chest wall platform and translation stage, respectively. The sinusoidal motion cam was chosen for all uses of the Quasar. The oval-shaped acrylic body houses the lung insert (see Figure 2.16a). In addition to the phantom, a Quasar software application made it possible to import, edit, and run sinusoidal, patient-specific, or custom motion waveforms (see Figure 2.16b).

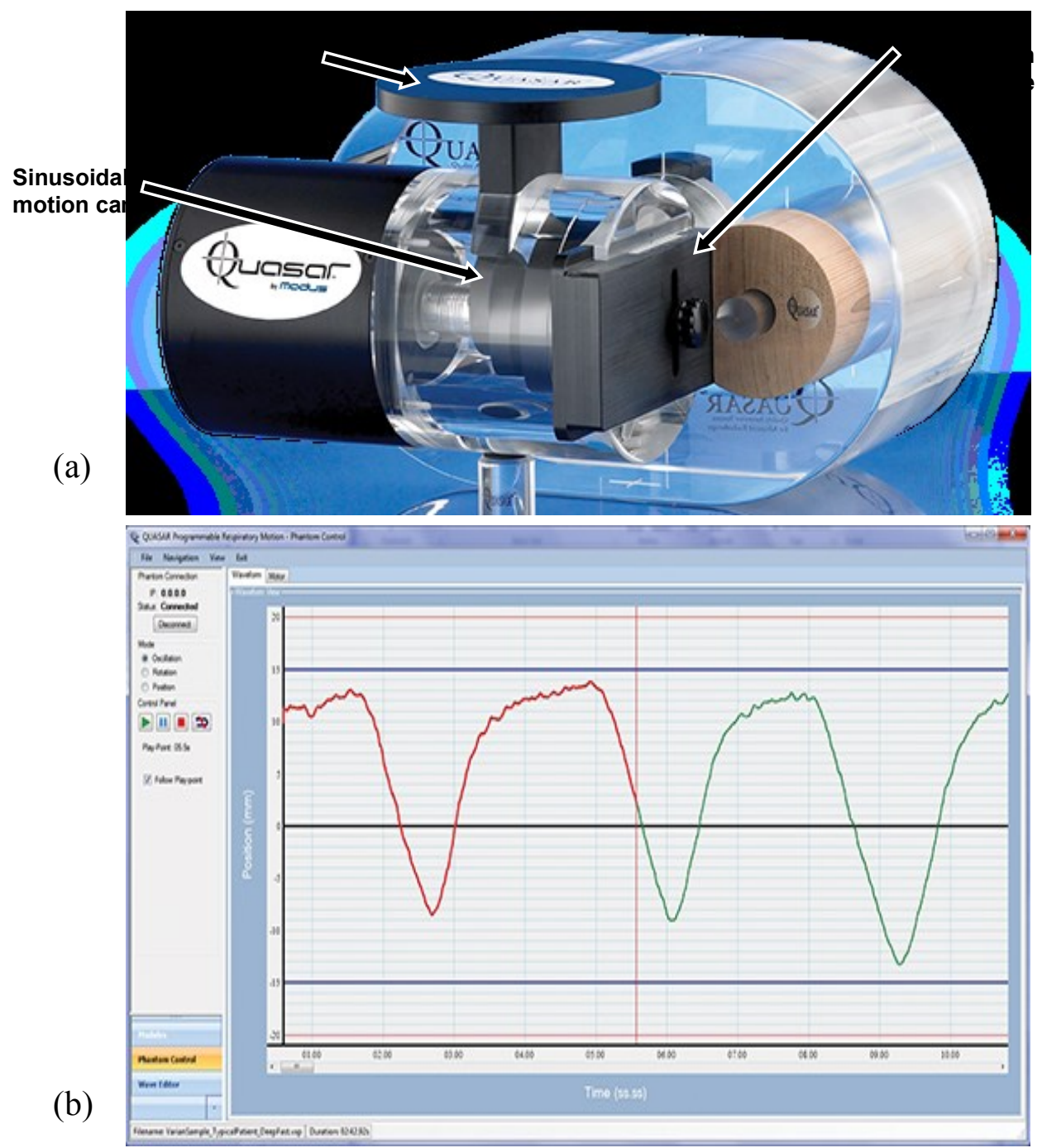

Figure 2.16 The Quasar Respiratory Motion Phantom (a) hardware and (b) software. Obtained with permission from Modus Medical Devices Inc. 


\subsubsection{Treatment Planning}

\subsubsection{Treatment Planning System}

The MultiPlan treatment planning system is software used to plan radiotherapy treatments on CyberKnife. There are four main steps (visualized as tabs in the upper part of Figure 2.17) in creating a plan: fuse, contour, align, and plan. A fifth step, visualize, simply displays the dose on the patient's anatomy. What follows is a brief description of each step in the context of how the plans were designed for the breast and lung experiments.

The fuse step involves aligning different image sets. For example, the CT scans of the phantom for static (Quasar off) conditions were fused to phases of the phantom for dynamic (Quasar on) conditions (i.e., 3DCT and 4DCT scans) as part of the lung experiment. The locations of the fiducials, which are radiopaque and clearly visible on the CT images, are identified and then the two images are 'registered' to one another by minimizing the distance between the selected points in the different image sets. Figure 2.17 demonstrates what occurs during the fuse step.

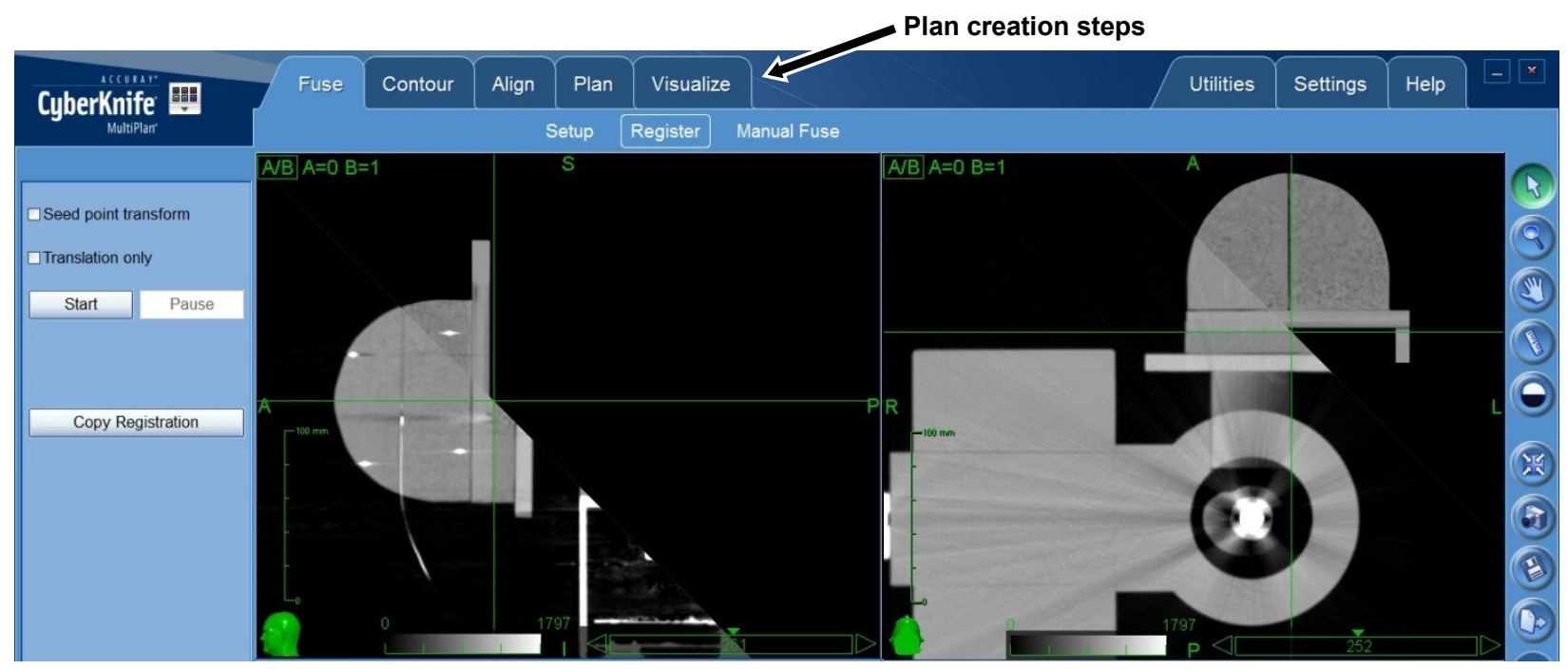

Figure 2.17 Screen capture of the MultiPlan treatment planning system's fuse step. In this case, a fusion of the CT image for the Quasar-breast phantom system with a small field of view, high resolution CT image of just the breast phantom is shown. 
The contour step consists of outlining and labeling various regions on the 3DCT image, such as adding GTV or PTV and OAR during patient planning. For the experiments in this work, this step included contouring the RADPOS detector (MOSFET and positioning sensor), the region to receive dose (e.g., Solid Water ball phantom) and streak artifacts due to the presence of high $\mathrm{Z}$ components in the CT. Several tools are available in order to simplify the contouring process, such as an 'auto interpolation' feature. This tool contours an object automatically over multiple CT image slices based on interpolating between contours of the same object in different image slices that came before and after in the superior/inferior direction. Figure 2.18 shows a part of the contouring step.

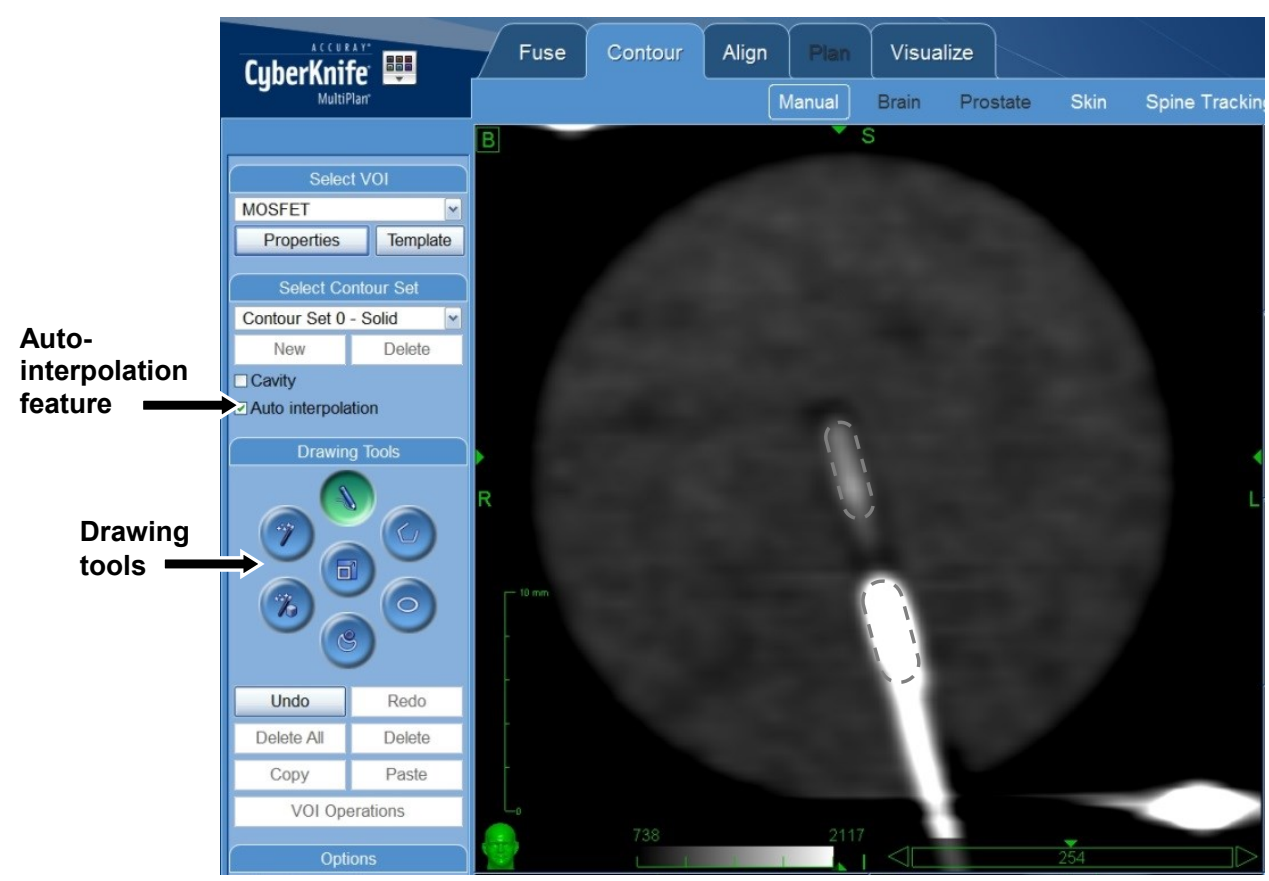

Figure 2.18 Screen capture of the MultiPlan treatment planning system's contour step. The MOSFET and position sensor of the RADPOS detector have been outlined. 
The align step deals with selecting the location of the imaging isocenter, setting various treatment parameters and precisely identifying the fiducial locations on the planning CT image. The different parameters which could be defined include the number of treatment fractions, the anatomy to be treated, the path of the robotic manipulator, the tracking method (e.g., Synchrony) to be used during treatment, and the collimator type (fixed or the Iris variable collimator). Figure 2.19 presents the various aspects of the align step.

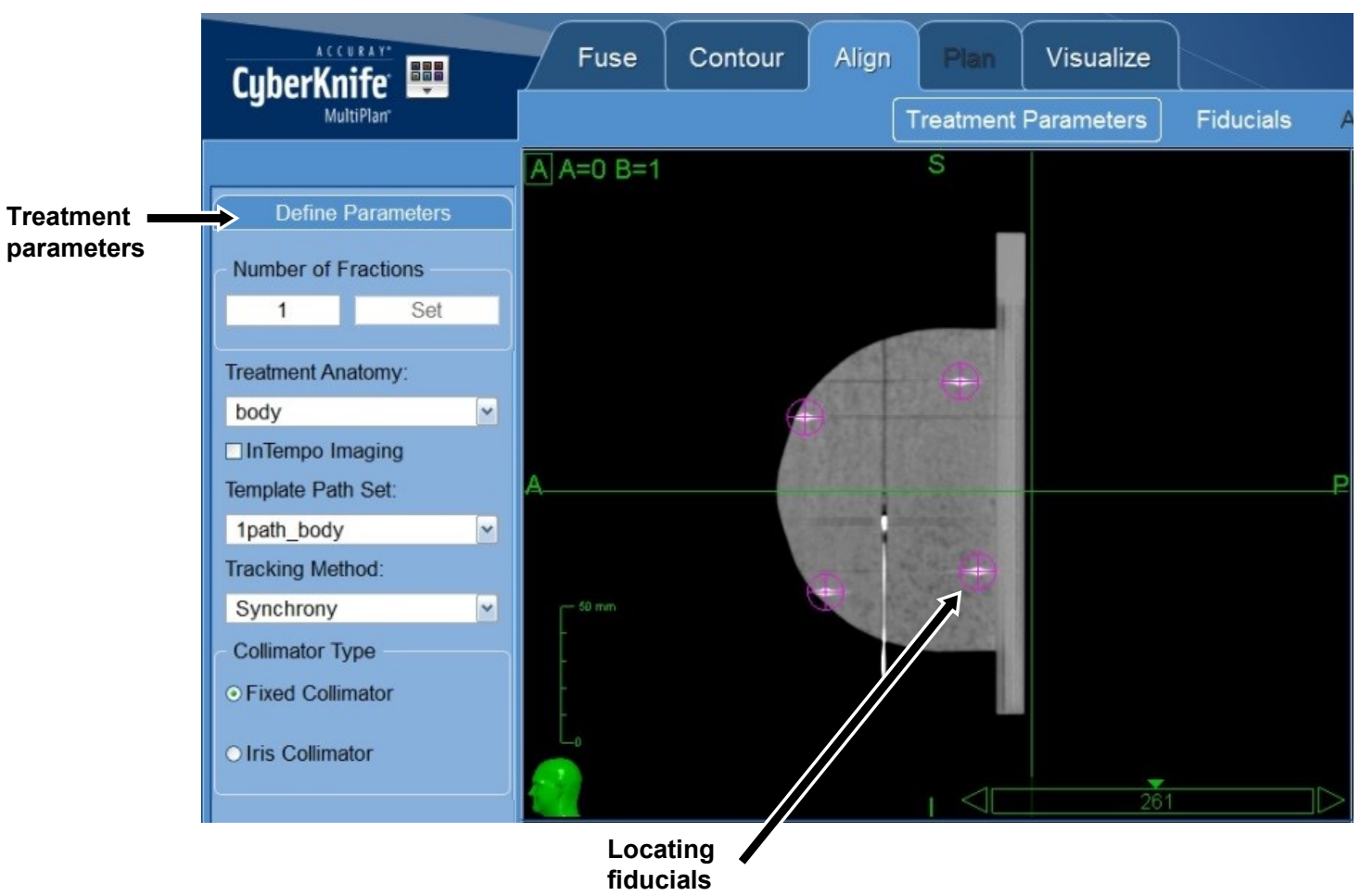

Figure 2.19 Screen capture of the MultiPlan treatment planning system's align step. The treatment parameters are being set and the locations of the fiducials have been identified. 
The plan step consists of treatment plan optimization and dose calculation. This step allows the user to assign density overrides, define the region over which the dose calculation takes place, compute the dose delivered per beam and optimize the beam weights to achieve various required dose limits and target constraints. The treatment time could then be further minimized through beam reduction and monitor unit reduction techniques. Figure 2.20 displays what the plan step looks like.

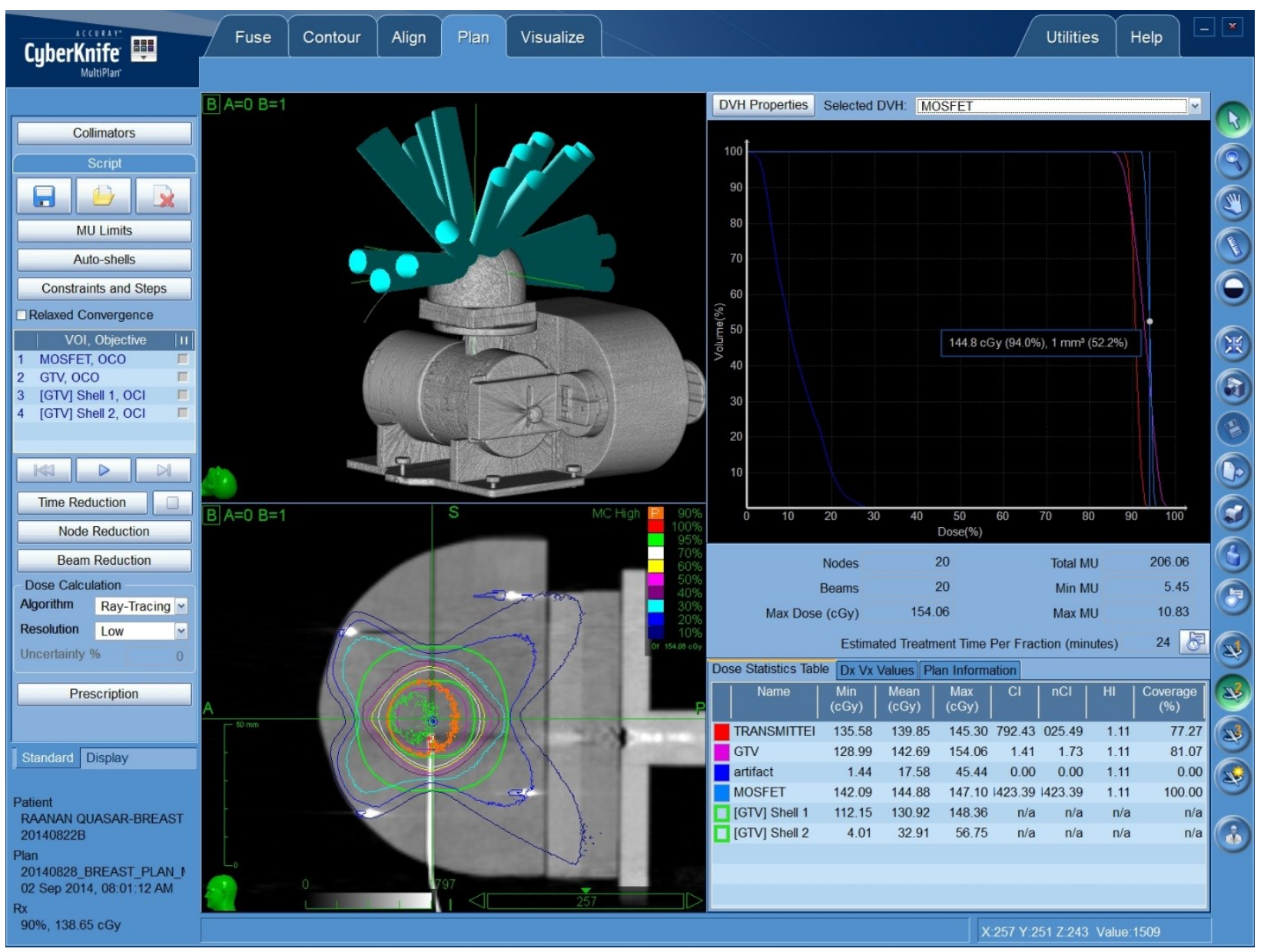

Figure 2.20 Screen capture of the MultiPlan treatment planning system's plan step. The spatial distribution of treatment beams are shown in the top-left and the dose distribution within the breast phantom is shown in the bottom-left. 


\subsubsection{Dose Calculations}

CyberKnife's treatment planning system performs dose calculations using two different algorithms: ray-tracing and Monte Carlo.

The ray-tracing algorithm performs an analytic dose calculation and computes the point dose within each voxel for every treatment beam. Heterogeneity corrections are implemented along the primary path of the treatment beam only using a radiological effective path length for the incident radiation. This effective path length is calculated by adding together the individual voxel contributions along the trajectory from the beam source to the target voxel using electron densities relative to water computed from the CT data [44]. However, ray-tracing does not account for how electron transport changes when the voxel-of-interest is surrounded by heterogeneous media [45]. Using beam data tables (derived from clinical measurements made in a water phantom) for tissue phantom ratios (TPR), off-centre ratios (OCR), and output factors (OF), the point dose per beam is calculated using ray-tracing from Equation 2.8:

$$
D / M U=O C R\left(\operatorname{coll}, R_{800}, D_{e f f}\right) \cdot\left(\frac{800}{S A D}\right)^{2} \cdot T P R\left(f, D_{e f f}\right) \cdot O F(\operatorname{coll}, S A D)
$$

where coll is the collimator size, $\mathrm{R}_{800}$ is the radial distance from central axis at $800 \mathrm{~mm}$ from the source, and $\mathrm{f}$ is the field-size projected at the calculation point [44].

The Monte Carlo algorithm performs a statistical dose calculation for a specified uncertainty level. It computes the dose within each voxel by taking into account how electron and photon interactions occur in heterogeneous media. As described by Ma et al. [46], the CyberKnife Monte Carlo dose calculation software performs computations in a 3D voxel geometry which models the heterogeneous anatomy of patients. A different 
material with variable density can be associated with every voxel, but the stopping power density correction for the material does not change.

Higher algorithm efficiency is achieved using pre-computed particle interaction and track data. This data includes photon mean free paths in prevalent anatomical materials (air, tissue, bone and water) for different energies and information for secondary electron tracks in water. These pre-simulated electron tracks, which have their step length resized in proportion to the local material density and stopping and scattering power ratios, are used to simulate electrons resulting from photon interactions [47]. Based on the fraction of the equivalent step length in a voxel, the energy deposition, and in turn the dose, may be calculated.

Since Monte Carlo calculations simulate the physical interactions that result in dose being deposited by photons and charged particles and absorbed by the medium, Monte Carlo is favored over ray-tracing for cases involving anatomical regions of significant heterogeneity [48].

In this work, Monte Carlo calculation parameters were always set to high resolution (i.e., smallest voxel size corresponding to the planning CT voxel size) and 1\% statistical uncertainty. 


\subsection{X-ray Imaging Dose}

\subsubsection{Overview}

Non-isocentric treatment plans are inherently more complex and generally require longer treatment times when compared to isocentric treatment plans. As a result, the patient's breathing pattern must be monitored over a longer duration, meaning that more CyberKnife imaging x-rays must be taken over the course of radiotherapy treatment (which could take several fractions), resulting in a larger imaging dose to the patient.

All four treatment cases of the breast experiment incorporated isocentric plans, consisting of approximately 33 x-ray pairs on average. The lung experiment incorporated three isocentric plan treatment cases and two non-isocentric plan treatment cases. Each isocentric plan treatment case consisted of approximately $40 \mathrm{x}$-ray pairs on average. Each non-isocentric plan treatment case consisted of approximately $80 \mathrm{x}$-ray pairs on average. An x-ray imaging dose experiment was designed in order to obtain an estimate of the imaging dose during prolonged CyberKnife treatments.

\subsubsection{Estimate of Dose Per X-ray Pair}

Calculations were done in order to determine a rough estimate of imaging x-ray dose for the breast phantom and lung insert. First, mass densities $(\rho)$ and mass attenuation coefficients $(\mu / \rho)$ were identified for materials making up the breast phantom and Quasarlung insert (see Table 2.1). Next, based on a CyberKnife imaging dose white paper [49], as well as the x-ray parameters used during the breast and lung experiments, an approximate unattenuated surface dose per x-ray was determined for each experiment's geometry (see Table 2.2). Finally, the thicknesses of the various materials along the x-ray 
imaging beams central axes for the breast and lung experiments were measured to assess the attenuation of x-rays at the position of the RADPOS detector active volume using Equation 2.9 (see Figure 2.21 and Figure 2.22).

Table 2.1 Materials making up the breast phantom and Quasar-lung insert setup, along with their associated mass densities and mass attenuation coefficients for an energy of $27 \mathrm{keV}$ (approximately $1 / 3$ of $80 \mathrm{kVp}$ ) and $40 \mathrm{keV}$ (approximately $1 / 3$ of $130 \mathrm{kVp}$ ).

\begin{tabular}{ccccc}
\hline $\begin{array}{c}\text { Average Photon } \\
\text { Energy (keV) }\end{array}$ & Phantom & Material & $\boldsymbol{\rho}\left(\mathbf{g} / \mathbf{c m}^{\mathbf{3}}\right)$ & $\boldsymbol{\mu} / \mathbf{\rho}\left(\mathbf{c m}^{2} / \mathbf{g}\right)$ \\
\hline 27 & Breast & Solid Water & 1.0 & 0.506 \\
40 & Breast & Solid Water & 1.0 & 0.268 \\
40 & Quasar & Acrylic (PMMA) & 1.14 & 0.235 \\
40 & Lung Insert & Cedar Wood & 0.26 & $0.236^{*}$ \\
\hline *based on approximate chemical composition of wood $(6 \% \mathrm{H}, 43 \% \mathrm{O}, 50 \% \mathrm{C}[50])$ \\
$\left(\frac{\mu}{\rho}\right)_{\text {wood }}^{40 \mathrm{keV}}$ \\
$=\left[0.06 \times\left(\frac{\mu}{\rho}\right)_{H}^{40 \mathrm{keV}}\right]+\left[0.43 \times\left(\frac{\mu}{\rho}\right)_{O}^{40 \mathrm{keV}}\right]+\left[0.50 \times\left(\frac{\mu}{\rho}\right)_{\mathrm{C}}^{40 \mathrm{keV}}\right]$ \\
$=$ \\
$=0.236 \frac{\mathrm{cm}^{2}}{\mathrm{~g}}$
\end{tabular}

Table 2.2 Surface dose per single image (from CyberKnife white paper [49]). In addition, approximate unattenuated dose per $x$-ray values, based on $x$-ray parameters used during the breast and lung experiments, are given in italics.

\begin{tabular}{ccccc}
\hline $\mathbf{k V p}$ & $\mathbf{m A}$ & $\mathbf{m s}$ & $\mathbf{m A s}$ & Dose (mGy) \\
\hline 100 & 100 & 100 & 10 & 0.15 \\
100 & 320 & 100 & 32 & 0.50 \\
120 & 100 & 100 & 10 & 0.24 \\
120 & 320 & 100 & 32 & 0.80 \\
150 & 100 & 100 & 10 & 0.38 \\
150 & 320 & 100 & 32 & 1.24 \\
80 & 250 & 80 & 20 & $\sim 0.30$ \\
130 & 200 & 80 & 16 & $\sim 0.62$ \\
\hline
\end{tabular}

$$
e^{-\mu \cdot x}=e^{-\left(\frac{\mu}{\rho}\right) \cdot \rho \cdot x}
$$




\subsubsection{Estimate for Breast Experiment}

Attenuation:

$e^{-\left(\frac{\mu}{\rho}\right) \cdot \rho \cdot x}=e^{-\left[\left(0.506 \frac{\mathrm{cm}^{2}}{\mathrm{~g}} \times 1.0 \frac{\mathrm{g}}{\mathrm{cm}^{3}} \times 4.4 \mathrm{~cm}\right)\right]}=0.108$

Total:

Dose $_{\text {Xray pair }}=(0.30 \mathrm{mGy} \times 0.108) \times 2=0.065 \mathrm{mGy}$

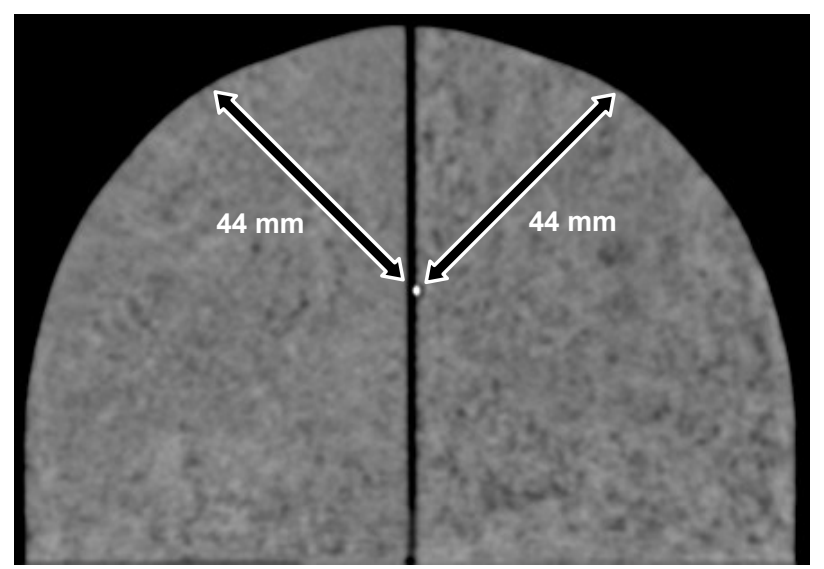

Figure 2.21 CT image of medial slice through the breast phantom. Shown is the measured thickness of the Solid Water material through which the imaging $x$-rays must pass through to reach the RADPOS MOSFET dosimeter.

\subsubsection{Estimate for Lung Experiment}

Side A attenuation:

$$
\begin{aligned}
& e^{-\left(\frac{\mu}{\rho}\right) \cdot \rho \cdot x} \\
= & e^{-\left[\left(0.235 \frac{\mathrm{cm}^{2}}{\mathrm{~g}} \times 1.14 \frac{\mathrm{g}}{\mathrm{cm}^{3}} \times 2.2 \mathrm{~cm}\right)+\left(0.236 \frac{\mathrm{cm}^{2}}{\mathrm{~g}} \times 0.26 \frac{\mathrm{g}}{\mathrm{cm}^{3}} \times 2.5 \mathrm{~cm}\right)+\left(0.268 \frac{\mathrm{cm}^{2}}{\mathrm{~g}} \times 1.0 \frac{\mathrm{g}}{\mathrm{cm}^{3}} \times 1.5 \mathrm{~cm}\right)\right]} \\
= & 0.318
\end{aligned}
$$

Side B attenuation:

$$
\begin{aligned}
& e^{-\left(\frac{\mu}{\rho}\right) \cdot \rho \cdot x} \\
= & e^{-\left[\left(0.235 \frac{\mathrm{cm}^{2}}{\mathrm{~g}} \times 1.14 \frac{\mathrm{g}}{\mathrm{cm}^{3}} \times 10 \mathrm{~cm}\right)+\left(0.236 \frac{\mathrm{cm}^{2}}{\mathrm{~g}} \times 0.26 \frac{\mathrm{g}}{\mathrm{cm}^{3}} \times 2.5 \mathrm{~cm}\right)+\left(0.268 \frac{\mathrm{cm}^{2}}{\mathrm{~g}} \times 1.0 \frac{\mathrm{g}}{\mathrm{cm}^{3}} \times 1.5 \mathrm{~cm}\right)\right]} \\
= & 0.039
\end{aligned}
$$

Total:

$$
\begin{aligned}
\text { Dose }_{\text {Xray pair }} & =\text { Dose }_{\text {Side A }}+\text { Dose }_{\text {Side } B} \\
& =(0.62 \mathrm{mGy} \times 0.318)+(0.62 \mathrm{mGy} \times 0.039) \\
& =0.222 \mathrm{mG})
\end{aligned}
$$




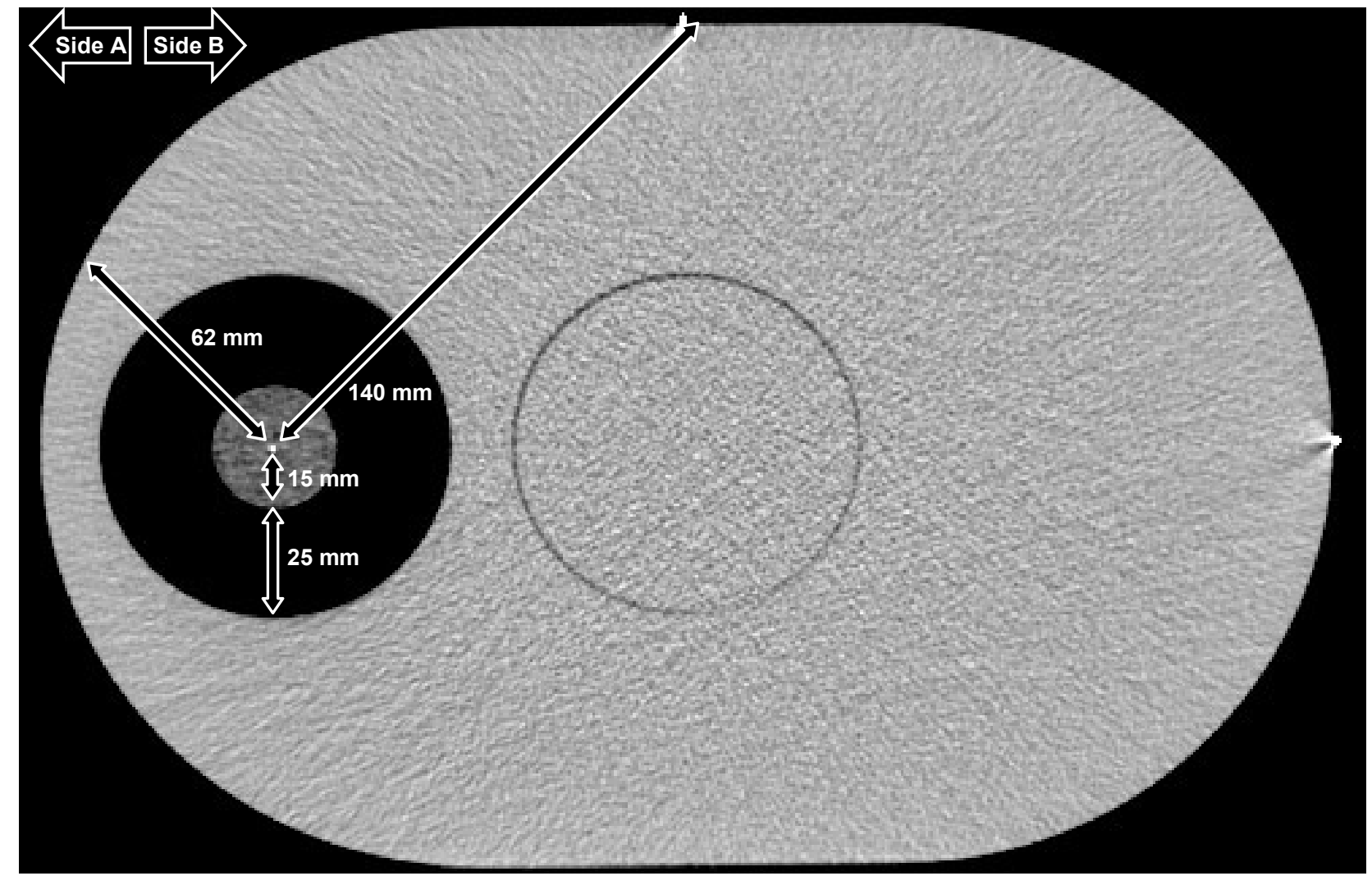

Figure 2.22 CT image of medial slice through the Quasar. Shown are the measured thicknesses of the various materials through which the imaging $x$-rays must pass through to reach the RADPOS MOSFET dosimeter.

\subsubsection{Experimental Design and Setup}

The experiment involved the same setup as the one shown in the left-hand part of Figure $2.25 \mathrm{~b}$, where the RADPOS probe and two films were inserted into the lung insert in between the Solid Water ball, all of which was housed within the acrylic body of the Quasar. A total x-ray dose of 10-15 cGy was chosen to be delivered as a compromise between experiment duration and signal-to-noise ratio. Therefore, $600 \mathrm{x}$-ray image pairs were to be taken, which would result in a total delivered dose of approximately $13 \mathrm{cGy}$ (according to the estimates from the previous section). 
Cross-calibration of the RADPOS and film was done immediately after the experiment. RADPOS (high sensitivity TN-1002RDM microMOSFET, SN 0669) and films were calibrated using an Exradin A1SL ion chamber (SN XW132493) and a Fluke 35040 electrometer ( $\mathrm{SN}$ 59947). The chamber cavity temperature was $21.9^{\circ} \mathrm{C}$ and the room pressure was $748.2 \mathrm{mmHg}$.

Ion chamber (Figure 2.23a) readings were performed in Solid Water at a depth of $5 \mathrm{~cm}$ for an $80 \mathrm{~cm}$ SAD setup. RADPOS (Figure 2.23b) and film (Figure 2.23c) crosscalibration readings were done at a depth of $5 \mathrm{~cm}$, with the ion chamber moved to a depth of $8 \mathrm{~cm}$ to monitor output variations of the CyberKnife LINAC.

(a)

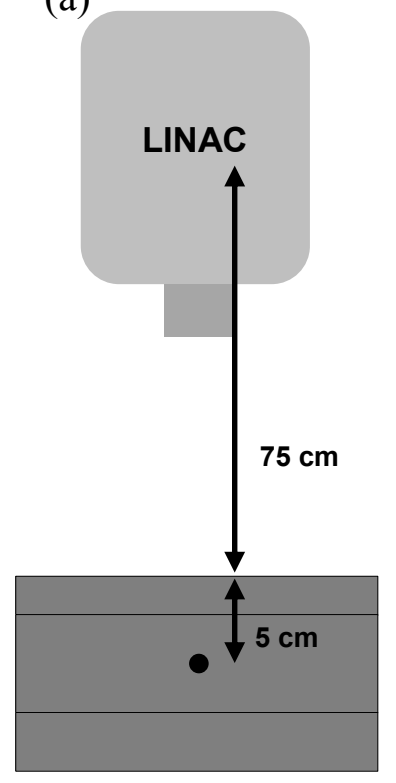

(b)

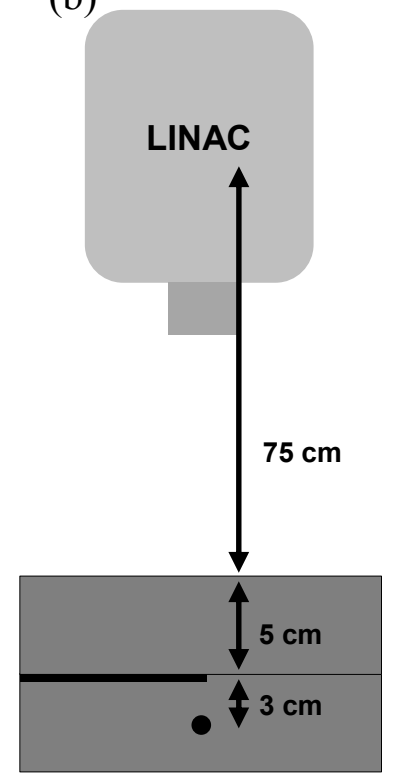

(c)

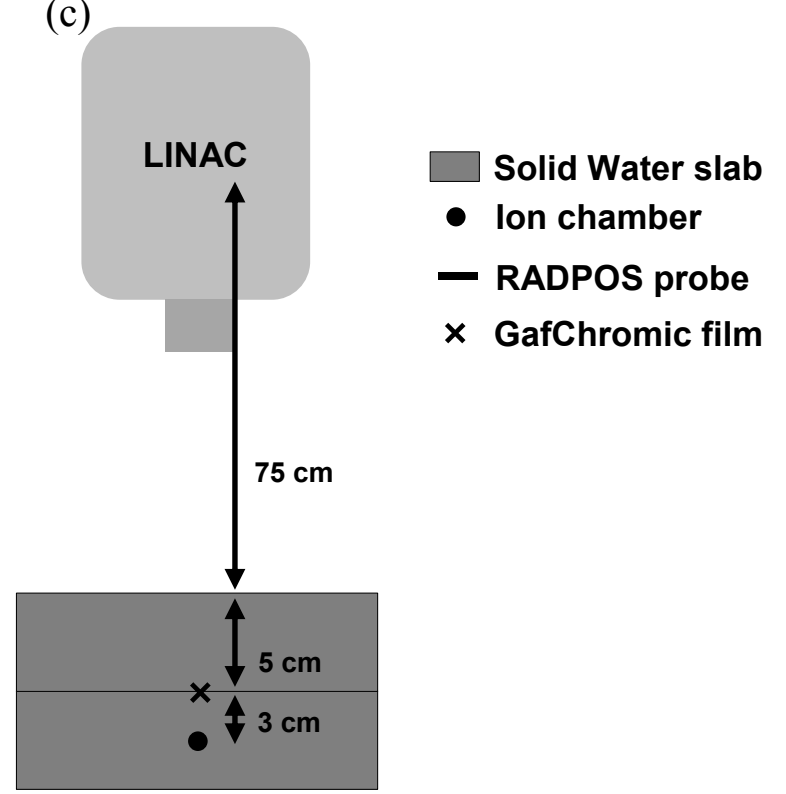

Figure 2.23 Cross-calibration conditions used for the (a) ion chamber readings, (b) RADPOS calibration, and (c) film calibration during the $x$-ray imaging dose experiment. 


\subsection{Breast Experiment}

\subsubsection{Apparatus and Setup}

The phantom was positioned on the chest wall platform of the Quasar phantom, to which three Synchrony LED optical markers were also attached (see Figure 2.24). The RADPOS was set to a position logging rate of $10 \mathrm{~Hz}$ (period of $100 \mathrm{~ms}$ ) to match the stereoscopic camera sampling rate of $25 \mathrm{~Hz}$ as closely as possible, while the Quasar was programmed to have its platform move sinusoidally ( $1 \mathrm{~cm}$ amplitude, $3 \mathrm{~s}$ period) in the anterior/posterior direction.

There were four treatment cases over the course of the experiment: Case 1 involved irradiating two films simultaneously for a static phantom, while Cases 2, 3, and 4 involved irradiating one film at a time for a dynamic phantom. A summary of the treatment cases and their specific details are given in Table 2.3. The RADPOS probe was present within the breast phantom for all cases. For all cases, the treatment plan was isocentric, used a $30 \mathrm{~mm}$ diameter cone with 20 beams, the point of intersection was located at the RADPOS detector and the total MU was 206.06. Each case took approximately 20-25 minutes to execute: 10-15 minutes for irradiation and 10 minutes for pre- and post-irradiation setup.

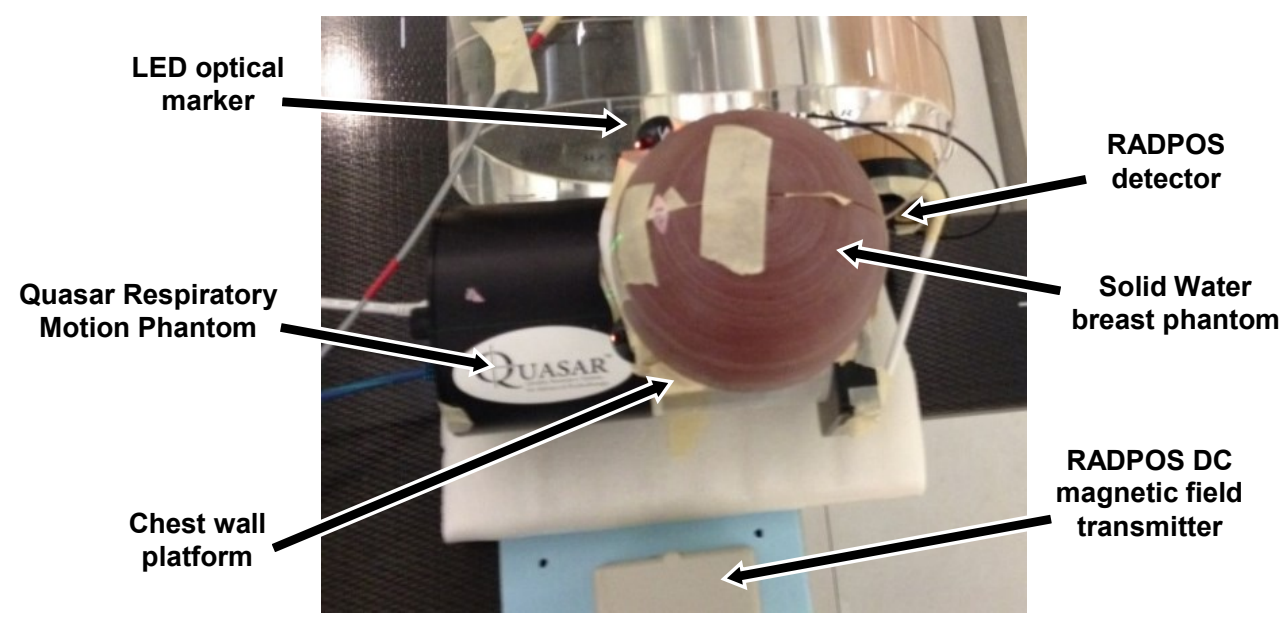

Figure 2.24 Apparatus and setup for the breast experiment. 
Table 2.3 Summary of the main details for the four breast experiment treatment cases.

\begin{tabular}{ccc}
\hline Case & Number of Films & Motion Type \\
\hline 1 & 2 & Static \\
2 & 1 & Sinusoidal \\
3 & 1 & Sinusoidal \\
4 & 1 & Sinusoidal \\
\hline
\end{tabular}

\subsubsection{Cross-Calibration}

Calibration and cross-calibration was done similarly to as described for the x-ray imaging dose experiment (see section 2.2.3). Specific differences included:

- RADPOS SN 0674

- Ion chamber cavity temperature was $22.15^{\circ} \mathrm{C}$ and the room pressure was 749.9 $\mathrm{mmHg}$

- RADPOS cross-calibration readings were done at a depth of $5 \mathrm{~cm}$, with the ion chamber at a depth of $9 \mathrm{~cm}$

- Film cross-calibration readings were done at a depth of $5 \mathrm{~cm}$, with the ion chamber at a depth of $8 \mathrm{~cm}$

\subsubsection{Treatment Planning}

A CT-based, isocentric treatment plan was created for the Solid Water breast phantom containing four metal fiducials and the RADPOS probe. Dose calculations were performed using the ray-tracing and Monte Carlo algorithms from CyberKnife's MultiPlan treatment planning system. 


\subsubsection{Positional Data Analysis}

For this experiment, the user selected a common time point from the LED and RADPOS motion signals based on matching common physical features in the waveforms (as shown in Figure 2.13). This allowed for temporal synchronization between the Synchrony and RADPOS data within the uncertainty of the temporal resolution of the RADPOS system.

\subsection{Lung Experiment}

\subsubsection{Apparatus and Setup}

The experiment was composed of five treatment cases, and two separate Quasar phantoms were used for most cases. One Quasar housed the lung insert, and another housed the chest wall platform (to which three LED markers were attached). Each case involved the irradiation of two films, which were inserted inside of the lung insert on top of the RADPOS probe prior to treatment delivery. The RADPOS was again set to a position logging rate of $10 \mathrm{~Hz}$. In addition, two treatment plan types were used: isocentric and non-isocentric. The isocentric plan used a $35 \mathrm{~mm}$ diameter cone with 28 beams, with the point of intersection located at the RADPOS detector and a total MU of 414.8. The non-isocentric plan used a $15 \mathrm{~mm}$ (43 beams) and a $30 \mathrm{~mm}$ (28 beams) diameter cone, with a total MU of 1147.5. Irradiation for isocentric plan cases took approximately 10-15 minutes, while irradiation for non-isocentric plan cases took approximately 20-25 minutes. In addition, setup for each case took about 20 minutes.

The five cases varied in complexity, and are summarized as follows: Case I0 involved a single, static Quasar. P0 was the same as I0, except that the treatment plan was 
changed to the more complex, non-isocentric patient plan. I1 involved a single Quasar with its lung insert (3.6 cm amplitude, superior/inferior direction) and chest platform (1 $\mathrm{cm}$ amplitude, anterior/posterior direction) components moving together sinusoidally (3.33 s period). I2 involved two Quasars, where the sinusoidal lung motion of one was out-of-phase (several tenths of a second) with the sinusoidal chest motion of the other. P1 was the most sophisticated case, where each Quasar's motion was set to an irregular waveform derived from a patient's log files. A non-isocentric plan was used, and the motions of the lung insert and chest platform were set to be out-of-phase. A summary of the treatment cases and their specific details is given in Table 2.4, and the experimental setups for the dynamic cases is shown in Figure 2.25. The RADPOS probe was present within the lung insert for all cases.

Table 2.4 Summary of the main details for the five lung experiment treatment cases.

\begin{tabular}{cccc}
\hline Case & Motion Type & Phase Type & Plan Type \\
\hline I0 & Static & N/A & Isocentric \\
P0 & Static & N/A & Non-Isocentric \\
I1 & Sinusoidal & In Phase & Isocentric \\
I2 & Sinusoidal & Out of Phase & Isocentric \\
P1 & Patient Waveform & Out of Phase & Non- Isocentric \\
\hline
\end{tabular}


(a)

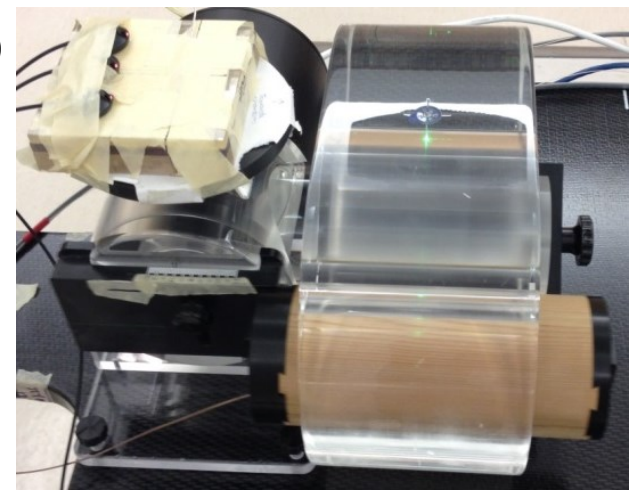

(b)

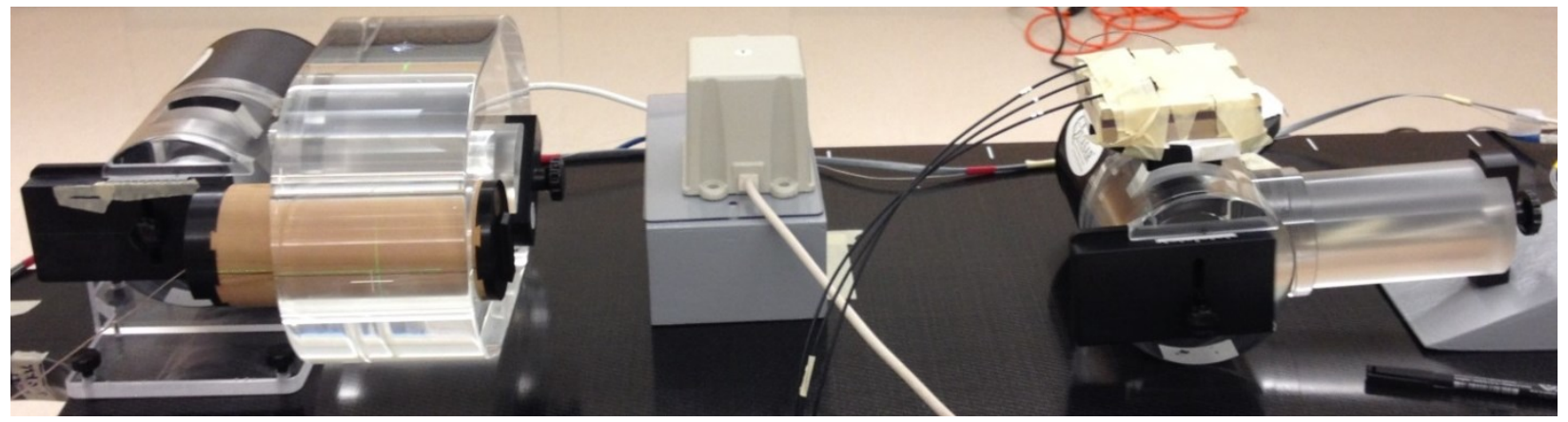

Figure 2.25 Setups for the dynamic cases of the lung experiment. For case (a) I1, the lung insert and chest platform (with the LEDs) both move together. For cases (b) I2 and P3, one Quasar has the insert and the other has the chest platform, which are made to move separately.

\subsubsection{Cross-Calibration}

Calibration and cross-calibration was done similarly to as described for the x-ray imaging dose experiment (see section 2.2.3). Specific differences included:

- $\quad$ RADPOS SN 0674 and SN 0669

- Ion chamber cavity temperature was $21.6^{\circ} \mathrm{C}$ and the room pressure was 760.6 $\mathrm{mmHg}$

- RADPOS and film cross-calibration readings were done at a depth of $5 \mathrm{~cm}$, with the ion chamber at a depth of $13 \mathrm{~cm}$ 


\subsubsection{Treatment Planning}

A more sophisticated approach was used for this experiment's treatment planning.

During CT scanning, two different scans were performed: a static (Quasar motion off) scan and a dynamic (Quasar motion on) scan; the dynamic scan was subsequently divided into ten phases, which covered the entire cycle of Quasar motion. To assess the effects of respiratory motion, dose calculations were done on three of the ten phases: the midrespiration phase (i.e., lung insert in the middle of the Quasar acrylic body), the maximum superior phase (i.e., lung insert protruding from the Quasar acrylic body in the superior direction as much as possible) and the maximum inferior phase (i.e., lung insert protruding from the Quasar acrylic body in the inferior direction as much as possible). Figure 2.26 shows illustrations of what the three phases-of-interest looked like relative to the Quasar acrylic body. This approach was taken for the isocentric and non-isocentric treatment delivery cases.
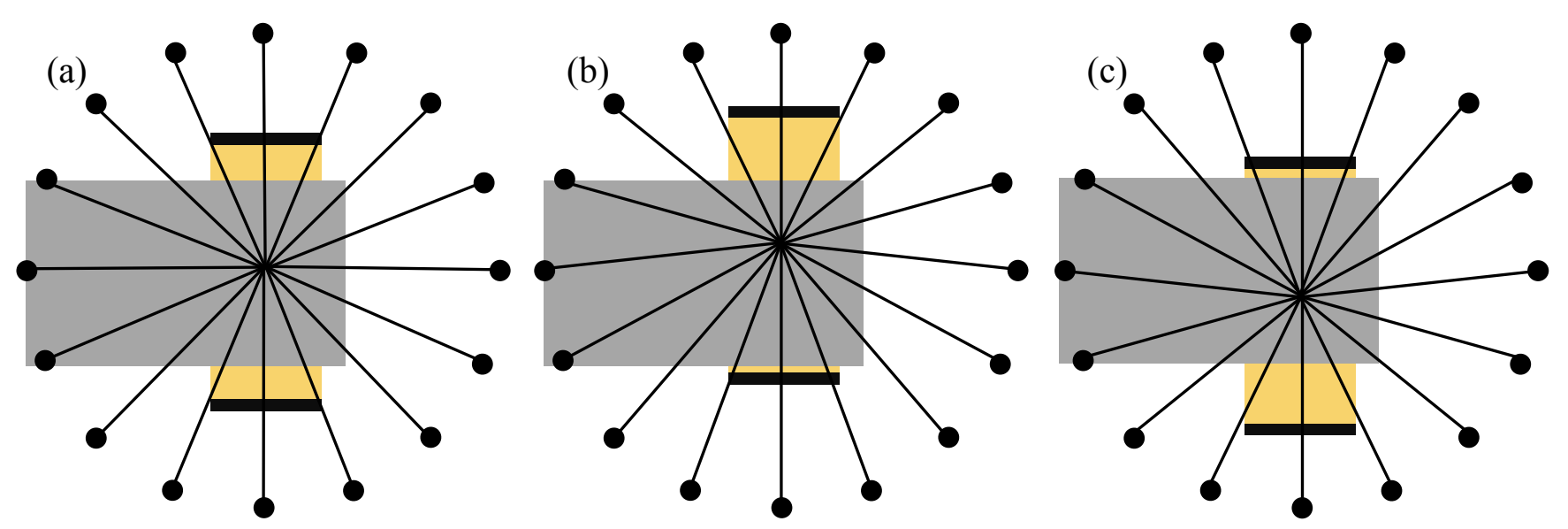

Figure 2.26 Illustration showing how the source positions (black circles) and beam directions (black lines) change for a moving target on the CyberKnife system. The middle (a) of respiration, as well as the maximum (b) superior and (c) inferior extents of Quasar respiratory motion are shown. In each case, the positions of the nodes remain constant but the direction the LINAC is pointing follows the Solid Water tumor within the lung insert. 


\subsubsection{Positional Data Analysis}

The temporal alignment for this experiment was almost the same as for the breast experiment. The only modification was that after the user had selected common point from the LED and RADPOS motion signals, an iterative optimization program was performed. This would search around the selected common time point for a slightly better temporal synchronization by slightly varying the calculated time difference between the Synchrony and RADPOS data. The time difference was varied from +1 to -1 seconds in steps of 0.01 seconds and the time difference which minimized the sum of the square of the differences between the motion signals was selected. 


\section{Results}

This chapter presents the quantitative findings of the breast and lung experiments, as well as the $\mathrm{x}$-ray imaging dose experiment. Corrections for $\mathrm{kV}$ imaging $\mathrm{x}$-ray dose are given for RADPOS and film. The dosimetric and positional results are presented for the breast and lung experiments. 


\subsection{X-ray Imaging Dose Experiment}

The various experimental details are given in Table 3.1, with the x-ray parameters exactly matching the conditions of the lung experiment.

Table 3.1 Details and parameters for $x$-ray imaging dose experiment.

\begin{tabular}{ccccc}
\hline \multirow{2}{*}{ \# of x-ray pairs } & \multicolumn{4}{c}{ X-ray Parameters } \\
\cline { 2 - 5 } & $\boldsymbol{k} \boldsymbol{V} \boldsymbol{m}$ & $\boldsymbol{m} \boldsymbol{A}$ & $\boldsymbol{m s}$ & $\boldsymbol{m} \boldsymbol{A s}$ \\
\hline 600 & 130 & 200 & 80 & 16 \\
\hline
\end{tabular}

\subsubsection{RADPOS}

Threshold voltage difference $(\Delta \mathrm{V})$ readings were performed before and after irradiation of the RADPOS (SN 0669) by the 600 x-ray pairs. The voltage measured prior to delivery of the x-rays was $14717.65 \mathrm{mV}$, and the voltage measured directly following delivery of the $\mathrm{x}$-rays was $15035.90 \mathrm{mV}$, yielding a voltage difference of $318.25 \mathrm{mV}$ due to the x-rays. Therefore, the voltage change per x-ray pair was determined to be 0.5304 $\mathrm{mV}$.

Since two RADPOS detectors were used over the course of the lung experiment, a simple rescaling was done (using the previously determined calibration coefficients of the detectors) with Equation 3.1 in order to determine the voltage per x-ray pair for the second RADPOS (SN 0674) with Equation 3.2:

$$
\begin{aligned}
& \frac{C F_{0674}}{C F_{0669}}=\frac{0.3613 \frac{c G y}{m V}}{0.3687 \frac{c G y}{m V}}=0.9799 \\
& \left(\Delta V / /_{\text {-ray pair }}\right)_{0674}=\frac{C F_{0674}}{C F_{0669}} \times(\Delta V / x \text {-ray pair })_{0669}
\end{aligned}
$$

This yielded an approximate voltage per x-ray pair of $0.5198 \mathrm{mV}$ for RADPOS 0674. 


\subsubsection{Film}

The film analysis protocol for this experiment was the same as that for the breast and lung experiments. Using the calibration curve for the $6 \mathrm{MV}$ photon beam, the total dose measured by the film over the course of the 600 x-rays was $28.48 \mathrm{cGy}$, which resulted in a dose per x-ray pair of $0.04747 \mathrm{cGy}$ in a ROI containing the MOSFET active volume.

\subsubsection{Correcting for X-ray Imaging Dose}

A summary of the x-ray imaging dose experiment is given in Table 3.2, with results showing the voltage/x-ray pair and dose/x-ray pair values determined for RADPOS and film, respectively.

Table 3.2 Summary of x-ray imaging dose experiment results.

\begin{tabular}{cccc}
\hline Detector & $\begin{array}{c}\text { 6 MV Calibration } \\
\text { Coefficient } \\
(\mathbf{c G y} / \mathbf{m V})\end{array}$ & $\begin{array}{c}\text { Voltage/x-ray pair } \\
(\mathbf{m V})\end{array}$ & $\begin{array}{c}\text { Dose/x-ray pair* } \\
\mathbf{( c G y )}\end{array}$ \\
\hline RADPOS $_{0674}$ & 0.3613 & 0.5198 & - \\
RADPOS $_{0669}$ & 0.3687 & 0.5304 & - \\
Film & - & - & 0.04747 \\
\hline
\end{tabular}

*using the $6 \mathrm{MV}$ photon beam calibration curve

In addition, based on the estimated and measured dose per x-ray values (for film) determined for the lung experiment setup, a measurement-to-estimate (ME) scaling factor was calculated and applied to the value determined in Equation 2.10. The result could then be applied to the measured film dose values of the breast experiment. 


$$
\begin{aligned}
& M E=\frac{\text { Dose }_{\text {Xray pair, } \text { measurement }}^{\text {Lung }}}{\text { Dose }}=\frac{0.04747 c G y}{0.022 c G y}=2.14 \\
& \text { Dose }_{\text {Xray pair,measurement }}^{\text {Breast }}=M E \times \text { Dose }_{\text {Xray pair, }, \text { estimate }}^{\text {Breast }} \\
& =2.16 \times 0.0065 c G y \\
& =0.0140 c G y
\end{aligned}
$$

Table 3.3 shows how the film dose measurements changed for the breast experiment (based on the estimate of Equation 3.4), while Table 3.4 and Table 3.5 show how the RADPOS voltage measurements and film dose measurements respectively changed for the lung experiment.

Table 3.3 Change in film dose measurements corrected for $x$-ray imaging dose during the breast experiment.

\begin{tabular}{cccc}
\hline Phantom & Uncorrected Dose (cGy) & Corrected Dose (cGy) & Dose Difference (cGy) \\
\hline Static & 147.50 & 147.04 & 0.46 \\
Dynamic & 145.31 & 144.86 & 0.45 \\
\hline
\end{tabular}

Table 3.4 Change in RADPOS voltage measurements corrected for $x$-ray imaging dose during the lung experiment.

\begin{tabular}{ccccc}
\hline Case & $\begin{array}{c}\text { RADPOS } \\
\text { SN }\end{array}$ & $\begin{array}{c}\text { Uncorrected Voltage } \\
(\mathbf{m V})\end{array}$ & $\begin{array}{c}\text { Corrected Voltage } \\
(\mathbf{m V})\end{array}$ & $\begin{array}{c}\text { Voltage } \\
\text { Difference }(\mathbf{m V})\end{array}$ \\
\hline I0 & 0674 & 668.37 & 654.86 & 13.51 \\
P0 & 0674 & 677.24 & 644.49 & 32.75 \\
I1 & 0674 & 668.56 & 655.57 & 12.99 \\
I2 & 0669 & 664.04 & 644.61 & 19.43 \\
P1 & 0669 & 706.90 & 666.59 & 40.31 \\
\hline
\end{tabular}


Table 3.5 Change in film dose measurements corrected for x-ray imaging dose during the lung experiment.

\begin{tabular}{cccc}
\hline Case & Uncorrected Dose (cGy) & Corrected Dose (cGy) & Dose Difference (cGy) \\
\hline I0 & 239.84 & 238.09 & 1.75 \\
P0 & 232.73 & 229.27 & 3.46 \\
I1 & 244.86 & 243.01 & 1.85 \\
I2 & 245.27 & 243.49 & 1.78 \\
P1 & 252.72 & 248.73 & 3.99 \\
\hline
\end{tabular}

For experimental cases that involved the isocentric plan (i.e., I0, I1, I2), the voltage difference due to $\mathrm{x}$-rays accounted for approximately $2-3 \%$ of the total uncorrected RADPOS voltage. Additionally, the dose difference due to x-rays accounted for approximately $0.7-0.8 \%$ of the total uncorrected film dose for these cases.

For experimental cases that involved the non-isocentric plan (i.e., P0, P1) which had a longer treatment time in which more $\mathrm{x}$-ray images were acquired, the voltage difference due to $\mathrm{x}$-rays accounted for approximately $5 \%$ of the total uncorrected RADPOS voltage. Additionally, the dose difference due to x-rays accounted for approximately $1.5 \%$ of the total uncorrected film dose for these cases.

\subsection{Breast Experiment}

\subsubsection{Dose Results}

Table 3.6 shows the calculated treatment planning system dose, as well as the measured RADPOS and average film dose values. The TPS calculated dose values were 146.7 cGy for ray tracing and $145.3 \mathrm{cGy}$ for Monte Carlo (1.0\% statistical uncertainty), with an additional dosimetric uncertainty of $0.5 \%$ for both resulting from TPS beam data. 
The right-hand columns of Table 3.6 give the gamma results for $3 \% / 1 \mathrm{~mm}$. Table 3.7 displays the percent differences when comparing the TPS, film, and RADPOS doses.

Shown in Figure 3.1a and Figure 3.1b are the dose profiles for the CyberKnife MultiPlan treatment planning system and the average film data, respectively. The profiles are for the breast phantom during beam delivery on a dynamic Quasar. The 2D gamma comparison for a 3\%/1 mm criteria is given in Figure 3.1c. Below, in Figure 3.1d and Figure 3.1e, are dose profile comparisons along the superior/inferior direction and the anterior/posterior direction, respectively.

Table 3.6 Dose comparison between TPS, film (corrected for imaging x-ray dose), and RADPOS, as well as the percent of film pixels passing a $3 \% / 1 \mathrm{~mm}$ gamma index criteria.

\begin{tabular}{cc|ccccc}
\hline \multicolumn{2}{c|}{} & \multicolumn{5}{c}{ Measured } \\
\hline $\begin{array}{c}\text { RT dose } \\
\text { (cGy) }\end{array}$ & $\begin{array}{c}\text { MC dose } \\
\text { (cGy) }\end{array}$ & Phantom & $\begin{array}{c}\text { RADPOS } \\
\text { dose (cGy) }\end{array}$ & $\begin{array}{c}\text { Film dose } \\
\text { (cGy) }\end{array}$ & $\begin{array}{c}\gamma \text {-index, } \\
\mathbf{3} \% / \mathbf{1 m m} \\
\text { (RT) }\end{array}$ & $\begin{array}{c}\boldsymbol{\gamma} \text {-index, } \\
\mathbf{3} / \mathbf{1 m m} \\
\text { (MC) }\end{array}$ \\
146.7 & $145.3 \pm 1.0 \%$ & Static & $147.2 \pm 1.6 \%$ & $147.0 \pm 1.9 \%$ & $97.2 \%$ & $96.7 \%$ \\
& & Dynamic & $149.6 \pm 1.4 \%$ & $144.9 \pm 1.8 \%$ & $97.3 \%$ & $97.0 \%$ \\
\hline
\end{tabular}

Table 3.7 Percent difference comparisons between TPS, film, and RADPOS dose values.

$$
\% \text { Difference }
$$

\begin{tabular}{cccccc}
\hline Phantom & RADPOS/RT & RADPOS/MC & Film/RT & Film/MC & RADPOS/Film \\
Static & $0.3 \%$ & $1.3 \%$ & $0.2 \%$ & $1.2 \%$ & $0.1 \%$ \\
Dynamic & $2.0 \%$ & $2.9 \%$ & $-1.3 \%$ & $-0.3 \%$ & $3.3 \%$ \\
\hline
\end{tabular}



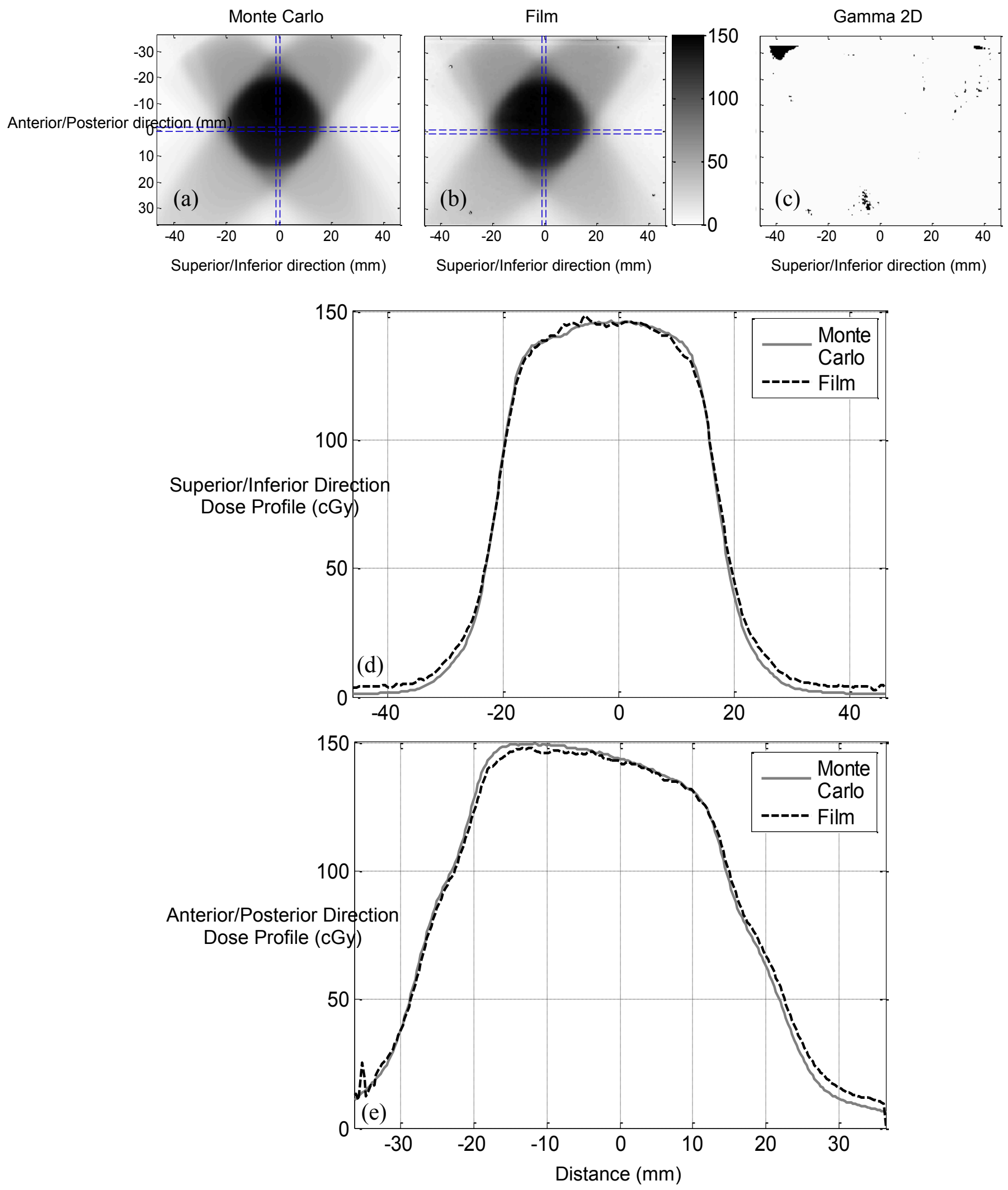

Figure 3.1 Sagittal dose planes within the moving breast phantom for (a) MultiPlan TPS and (b) the average film data, as well as (c) $2 \mathrm{D}$ gamma comparison for $3 \% / 1 \mathrm{~mm}$, which shows pixels which do not pass the gamma criteria as black. Also shown is a dose profile comparison along the directions: (d) superior/inferior, and (e) anterior/posterior (direction of phantom motion). 


\subsubsection{Position Results}

The plot in Figure 3.2 shows all the components of the three position tracking modalities after implementation of the coordinate alignment algorithm. The RADPOS and LED signals, represented by the solid curves and dashed curves, respectively, are shown as well as fiducial marker data points, represented by the dots. A zoomed-in version of the same plot, shown in Figure 3.3, demonstrates how the three position tracking modalities agreed at the fiducial's time points. The LED and RADPOS position data closely matched the fiducial data along the $\mathrm{z}$ direction (primary direction of motion), but agreement was worse along the $\mathrm{x}$ and $\mathrm{y}$ directions.

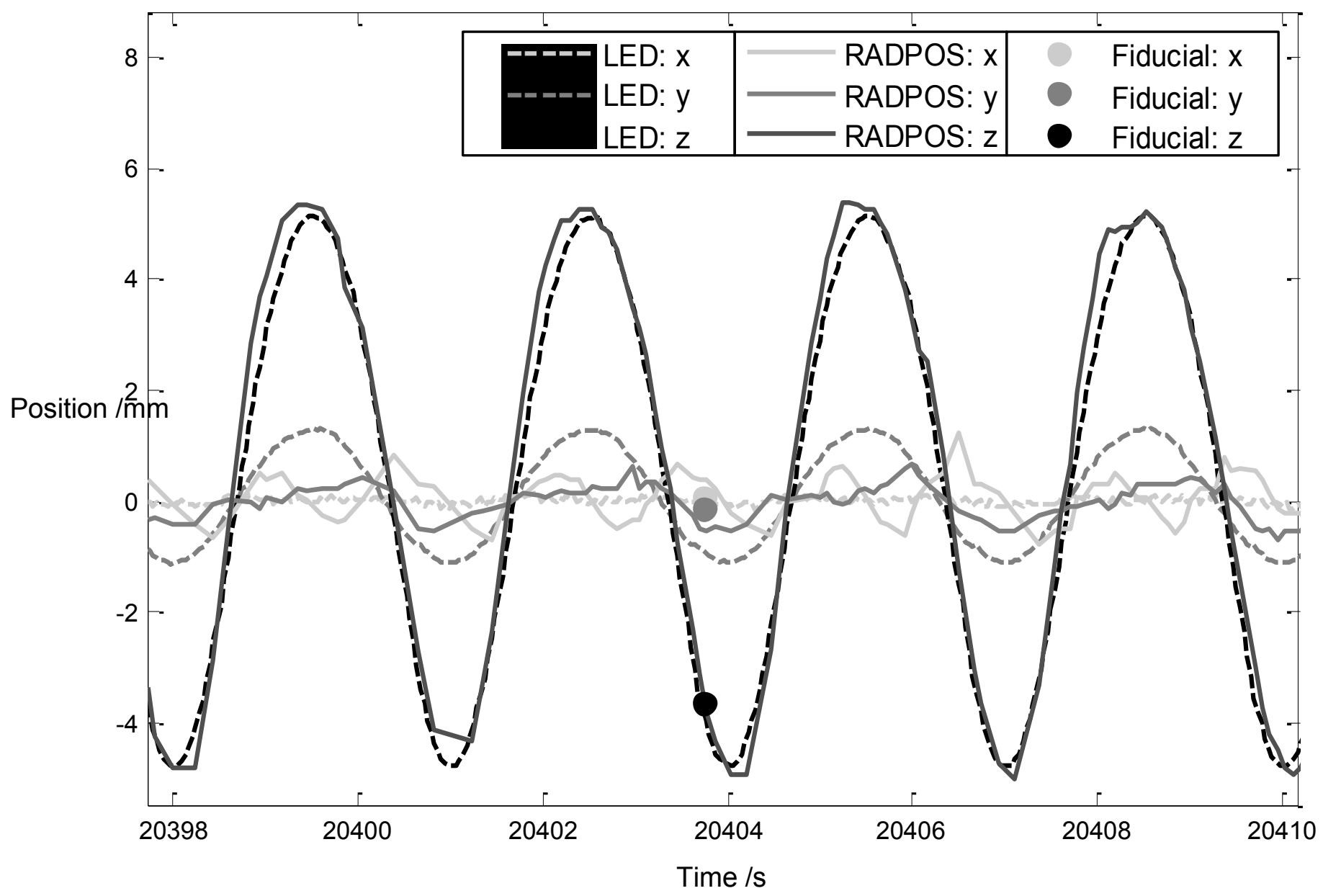

Figure 3.2 The LEDs, RADPOS, and fiducial position measurements along the $x, y$, and $z$ directions after implementation of the algorithm. 


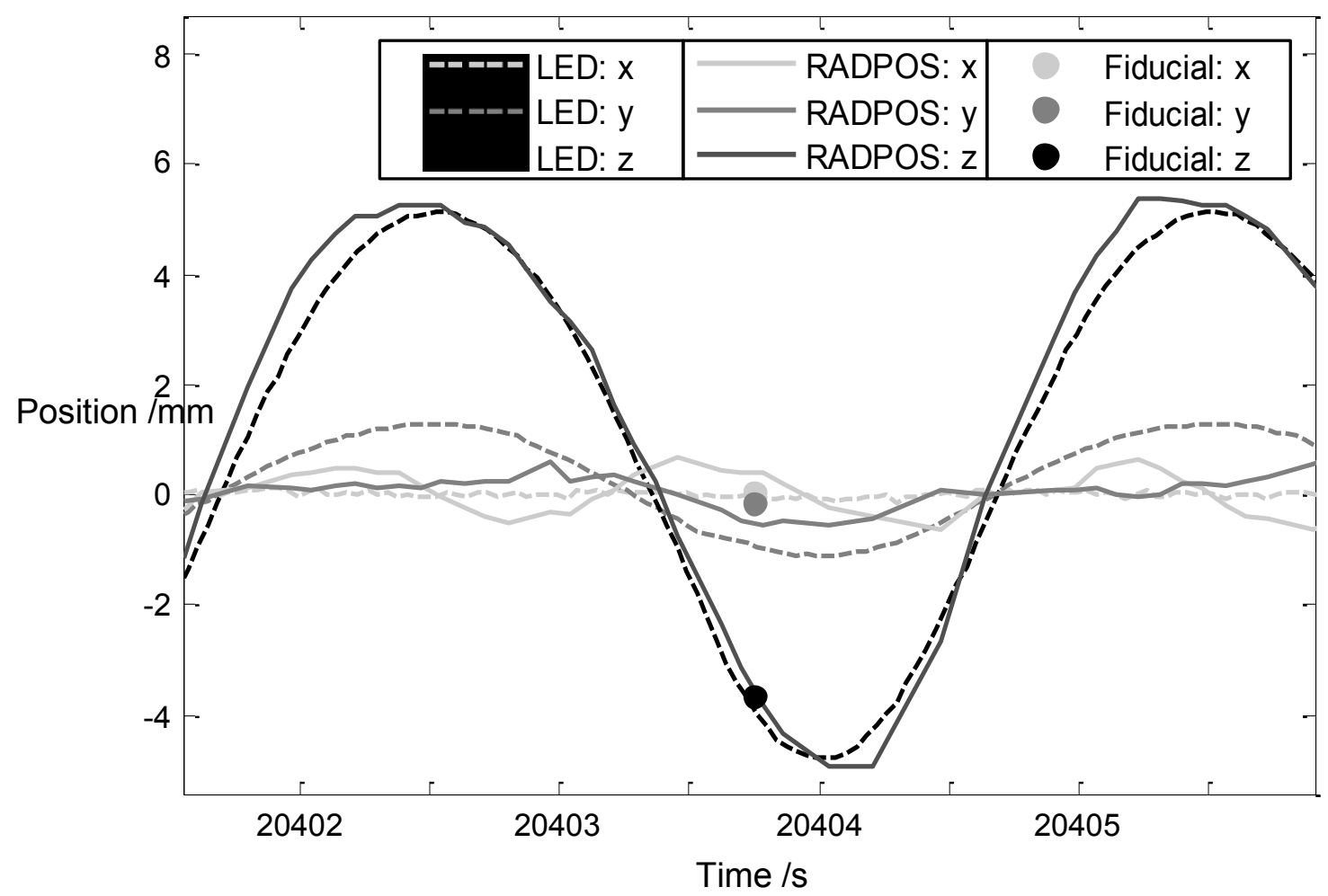

Figure 3.3 Closer view of a region of Figure 3.2, showing how the three position tracking modalities match up at the fiducial's time points.

Table 3.8 gives the quantitative results, comparing the LED, RADPOS and fiducial position measurements, as well as the correlation model position data. Overall, the LED and RADPOS measurements were in good agreement with each other and with fiducial measurements, with the average difference between any two position measurement datasets much less than $1 \mathrm{~mm}$ (see section 4.3 .2 for further discussion of this point). The standard deviation of their differences was $\leq 0.52 \mathrm{~mm}$ for all directions when averaged over the three dynamic cases. Similarly, the LED and RADPOS measurements were $\leq 0.51 \mathrm{~mm}$ average standard deviation when compared to each other. The standard deviation of the differences between the LEDS and Synchrony predictive model was $\leq 0.41 \mathrm{~mm}$ for all directions when averaged over the three dynamic cases. 
Between the RADPOS and model, the standard deviation of the differences was $\leq 0.63$ $\mathrm{mm}$ for all directions when averaged over the three dynamic cases.

Table 3.8 Quantitative positional results for the three dynamic treatment cases of the breast, comparing the LED, RADPOS, fiducial and model positional data. The primary direction of motion for all experimental cases is the $\mathrm{z}$ direction. Comparison 1 was performed in the fiducial time domain, whereas Comparison 2 was performed in either the RADPOS (LED-RADPOS, RADPOSModel) or Synchrony (LED-MODEL) time domains (see section 2.1.4.2).

Standard Deviation of the Differences ( $\mathrm{mm}$ )

\begin{tabular}{|c|c|c|c|c|c|c|}
\hline & \multirow{2}{*}{$\begin{array}{l}\text { Position } \\
\text { Datasets }\end{array}$} & \multirow{2}{*}{ Direction } & \multicolumn{4}{|c|}{ Dynamic Cases } \\
\hline & & & 2 & 3 & 4 & Average \\
\hline \multirow{6}{*}{ 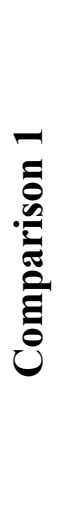 } & & $x$ & 0.08 & 0.07 & 0.07 & 0.07 \\
\hline & LED-Fid & $y$ & 0.48 & 0.47 & 0.62 & 0.52 \\
\hline & & $z$ & 0.19 & 0.20 & 0.18 & 0.19 \\
\hline & & $x$ & 0.23 & 0.25 & 0.39 & 0.29 \\
\hline & RADPOS-Fid & $y$ & 0.59 & 0.58 & 0.28 & 0.48 \\
\hline & & $z$ & 0.42 & 0.37 & 0.47 & 0.42 \\
\hline \multirow{9}{*}{ 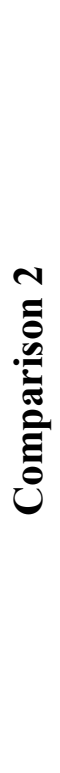 } & & $x$ & 0.25 & 0.28 & 0.40 & 0.31 \\
\hline & $L E D-R A D P O S$ & $y$ & 0.43 & 0.47 & 0.62 & 0.51 \\
\hline & & $z$ & 0.43 & 0.38 & 0.43 & 0.41 \\
\hline & & $x$ & 0.05 & 0.05 & 0.05 & 0.05 \\
\hline & LED-Model & $y$ & 0.58 & 0.43 & 0.23 & 0.41 \\
\hline & & $z$ & 0.06 & 0.16 & 0.16 & 0.12 \\
\hline & \multirow{3}{*}{ RADPOS-Model } & $x$ & 0.23 & 0.28 & 0.40 & 0.30 \\
\hline & & $y$ & 0.61 & 0.48 & 0.81 & 0.63 \\
\hline & & $z$ & 0.42 & 0.42 & 0.51 & 0.45 \\
\hline
\end{tabular}




\subsection{Lung Experiment}

\subsubsection{Dose Results}

Table 3.9 gives the treatment planning system dosimetric results for the max inferior, mid respiration and max superior lung insert positions computed at the RADPOS and film planes (see Figure 3.4). In addition, the measured dose values for RADPOS and film (all of which have been corrected for the additional dose acquired from imaging $\mathrm{x}-$ rays), along with their comparisons to the (mid respiration) TPS dose values and to each other, are given in Table 3.10, along with the gamma criteria analysis results for 3\%/1 $\mathrm{mm}$ and $3 \% / 2 \mathrm{~mm}$ agreement..

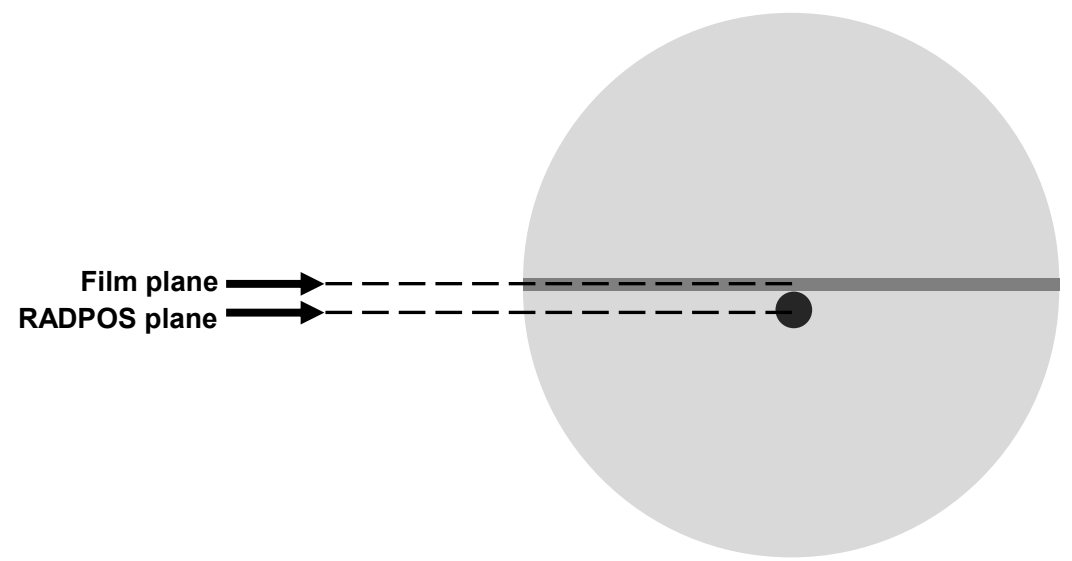

Figure 3.4 Illustration of cross-section through the lung insert, showing the film (gray bar) and RADPOS (black circle) positions inside of it. Also shown are the coronal planes for the film and RADPOS, which were used for TPS dose calculations.

The gamma analysis results for the isocentric plan cases (I0, I1, I2) were approximately $15 \%$ higher than those for the non-isocentric plan cases $(\mathrm{P} 0, \mathrm{P} 1)$ on average for $3 \% / 1 \mathrm{~mm}$ agreement. For the case of $3 \% / 2 \mathrm{~mm}$ agreement, the results for the isocentric plan cases were approximately $10 \%$ higher than those for the non-isocentric plan cases on average, with all pass rates being above $85 \%$. The RADPOS and film doses agreed with TPS values to within approximately $2 \%$ for all experimental cases except for P0, where the RADPOS and film doses were 3.6\% and 5.9\% lower than the TPS doses, 
respectively. Also, the measured RADPOS and film values were generally in good agreement, where differences between them did not exceed $3.4 \%$.

The $2 \mathrm{D}$ dose profiles for the treatment planning system and average film data for case I1 are shown in Figure 3.5a and Figure 3.5b, respectively, along with a gamma comparison in Figure 3.5c. Below, in Figure 3.5d and Figure 3.5e, are dose profile comparisons along $\mathrm{x}$ and $\mathrm{y}$ directions, respectively. A similar set of plots for case $\mathrm{P} 0$ are shown in Figure 3.6, where the gamma comparison was done for 3\%/2 mm agreement.

Table 3.9 TPS dose values at different phases of the respiratory cycle in the RADPOS plane and film plane.

\begin{tabular}{|c|c|c|c|c|c|c|}
\hline \multirow{2}{*}{ Case } & \multicolumn{3}{|c|}{$\begin{array}{l}\text { TPS Dose computed in the } \\
\text { RADPOS Plane }(c G y) \pm 1.0 \%\end{array}$} & \multicolumn{3}{|c|}{$\begin{array}{l}\text { TPS Dose computed in the } \\
\text { Film Plane }(c G y) \pm 1.0 \%\end{array}$} \\
\hline & $\begin{array}{c}\text { Max } \\
\text { Inferior }\end{array}$ & $\begin{array}{c}\text { Mid } \\
\text { Respiration }\end{array}$ & $\begin{array}{c}\text { Max } \\
\text { Superior }\end{array}$ & $\begin{array}{c}\text { Max } \\
\text { Inferior }\end{array}$ & $\begin{array}{c}\text { Mid } \\
\text { Respiration }\end{array}$ & $\begin{array}{c}\text { Max } \\
\text { Superior }\end{array}$ \\
\hline IO & 240.5 & 237.2 & 244.0 & 241.9 & 238.2 & 244.9 \\
\hline PO & 245.1 & 241.5 & 248.1 & 246.5 & 243.7 & 248.4 \\
\hline I1 & 240.5 & 237.2 & 244.0 & 241.9 & 238.2 & 244.9 \\
\hline$I 2$ & 240.5 & 237.2 & 244.0 & 241.9 & 238.2 & 244.9 \\
\hline$P 1$ & 245.1 & 241.5 & 248.1 & 246.5 & 243.7 & 248.4 \\
\hline
\end{tabular}

Table 3.10 Measured RADPOS and film (both corrected for imaging $x$-ray dose) dose values, as well as percent difference comparisons between RADPOS and mid respiration TPS

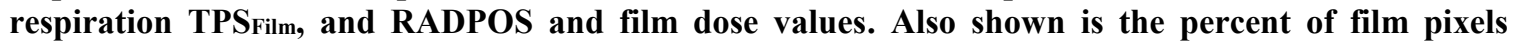
passing a $3 \% / 1 \mathrm{~mm}$ and $3 \% / 2 \mathrm{~mm}$ gamma index criteria.

\begin{tabular}{|c|c|c|c|c|c|c|c|}
\hline \multirow{2}{*}{ Case } & \multicolumn{3}{|c|}{ Measured Dose (cGy) } & \multicolumn{2}{|c|}{$\%$ Difference } & \multirow{2}{*}{$\begin{array}{c}\gamma \text {-index, } \\
\text { 3\%/1 mm }\end{array}$} & \multirow{2}{*}{$\begin{array}{c}\gamma \text {-index, } \\
3 \% / 2 \mathrm{~mm}\end{array}$} \\
\hline & $\begin{array}{c}\text { RADPOS } \\
\pm 1.6 \%\end{array}$ & $\begin{array}{c}\text { Film } \\
\pm 1.9 \%\end{array}$ & $\begin{array}{c}\text { RADPOS/ } \\
\text { Film }\end{array}$ & $\begin{array}{l}\text { RADPOS/ } \\
\text { TPSRADPOS }\end{array}$ & $\begin{array}{c}\text { Film/ } \\
\text { TPSFilm }\end{array}$ & & \\
\hline IO & 236.6 & 238.1 & $-0.6 \%$ & $-0.3 \%$ & $0.0 \%$ & $99.5 \%$ & $99.9 \%$ \\
\hline PO & 232.8 & 229.3 & $1.6 \%$ & $-3.6 \%$ & $-5.9 \%$ & $84.2 \%$ & $87.8 \%$ \\
\hline I1 & 236.8 & 243.0 & $-2.5 \%$ & $-0.2 \%$ & $2.0 \%$ & $98.8 \%$ & $99.7 \%$ \\
\hline $\mathrm{I} 2$ & 235.3 & 243.5 & $-3.4 \%$ & $-0.8 \%$ & $2.2 \%$ & $83.5 \%$ & $91.4 \%$ \\
\hline$P 1$ & 245.8 & 248.7 & $-1.2 \%$ & $1.8 \%$ & $2.1 \%$ & $73.7 \%$ & $85.5 \%$ \\
\hline
\end{tabular}



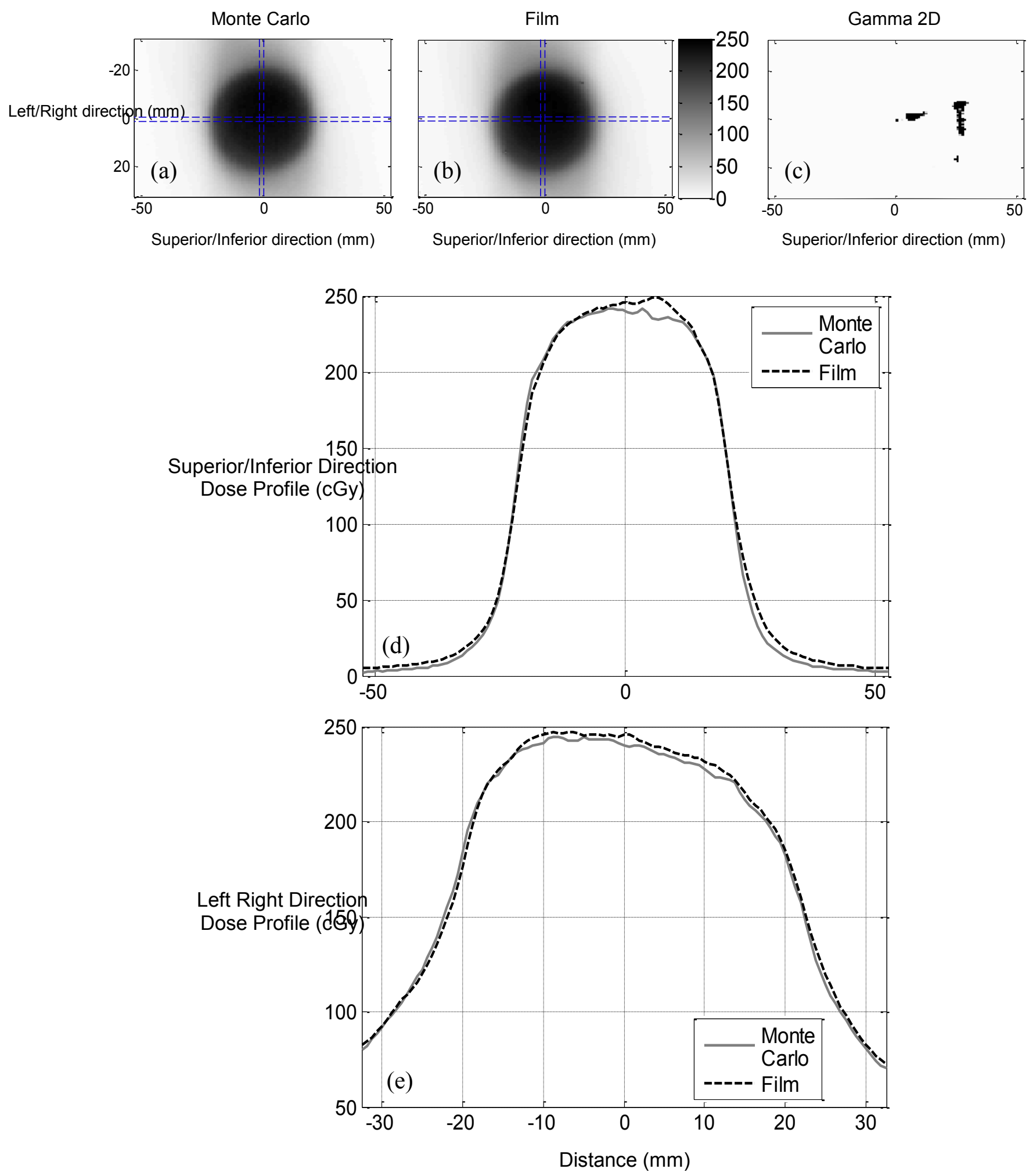

Figure 3.5 Dose profiles along the coronal plane within the lung insert (case I1) for (a) MultiPlan TPS and (b) the average film data, as well as (c) 2D gamma comparison for $3 \% / 1 \mathrm{~mm}$, which shows pixels which do not pass the gamma criteria as black. Also shown is a dose profile comparison along the directions: (d) superior/inferior (direction of phantom motion), and (e) left/right. 

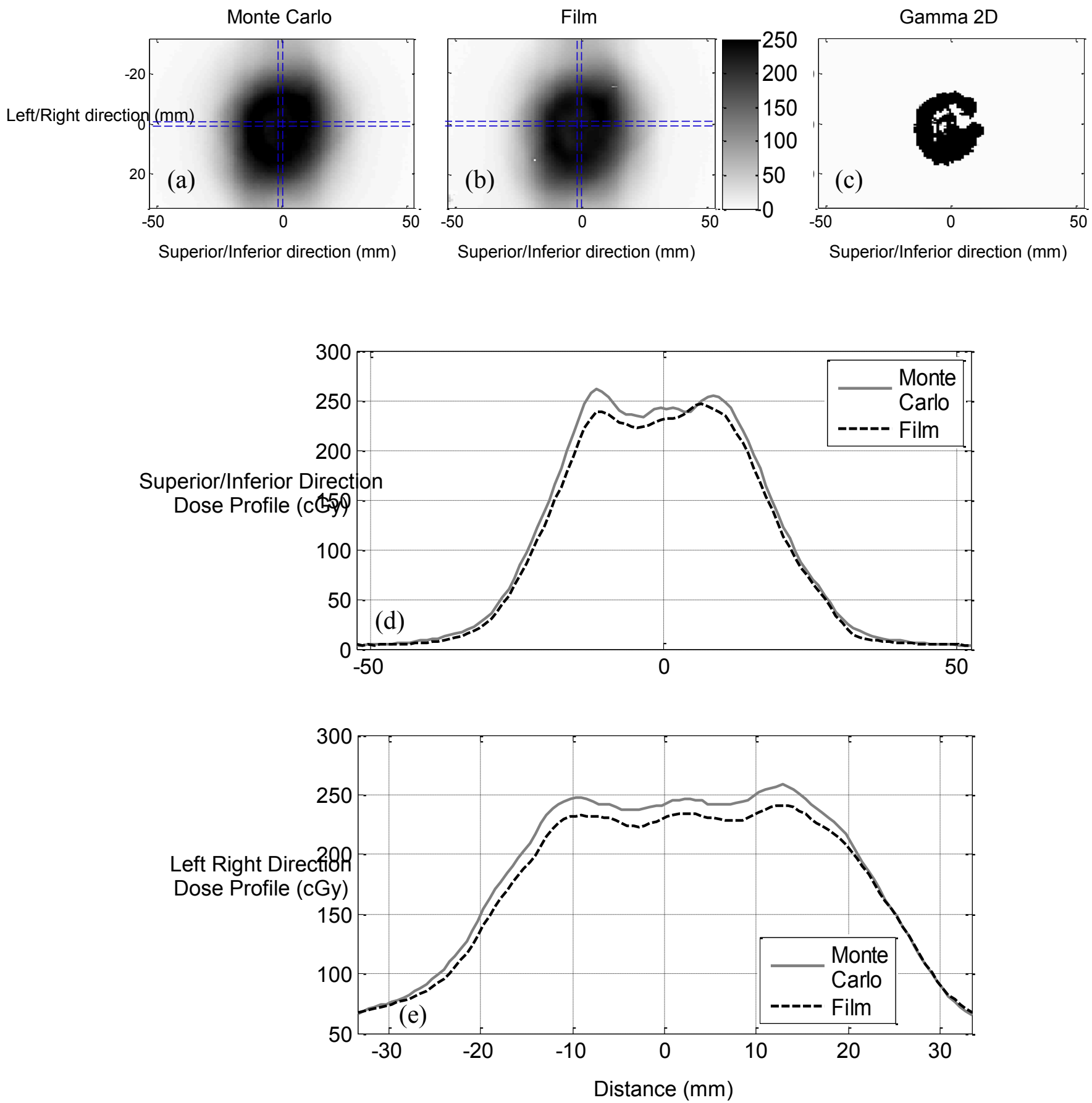

Figure 3.6 Dose profiles along the coronal plane within the lung insert (case P0) for (a) MultiPlan TPS and (b) the average film data, as well as (c) 2D gamma comparison for $3 \% / 2 \mathrm{~mm}$, which shows pixels which do not pass the gamma criteria as black. Also shown is a dose profile comparison along the directions: (d) superior/inferior (direction of phantom motion), and (e) left/right. 


\subsubsection{Position Results}

The RADPOS measurements were compared to fiducial positions, as well as to CyberKnife's predictive correlation model. The plots in Figure 3.7 show the model, RADPOS, and fiducials after implementation of the coordinate alignment algorithm for the primary direction of motion for the three dynamic cases of the experiment: case I1 in Figure 3.7a, case $\mathrm{I} 2$ in Figure 3.7b, and case P1 in Figure 3.7c. The RADPOS and model signals are represented by the solid curves and dashed curves, respectively, and the fiducials points are represented by the dots.

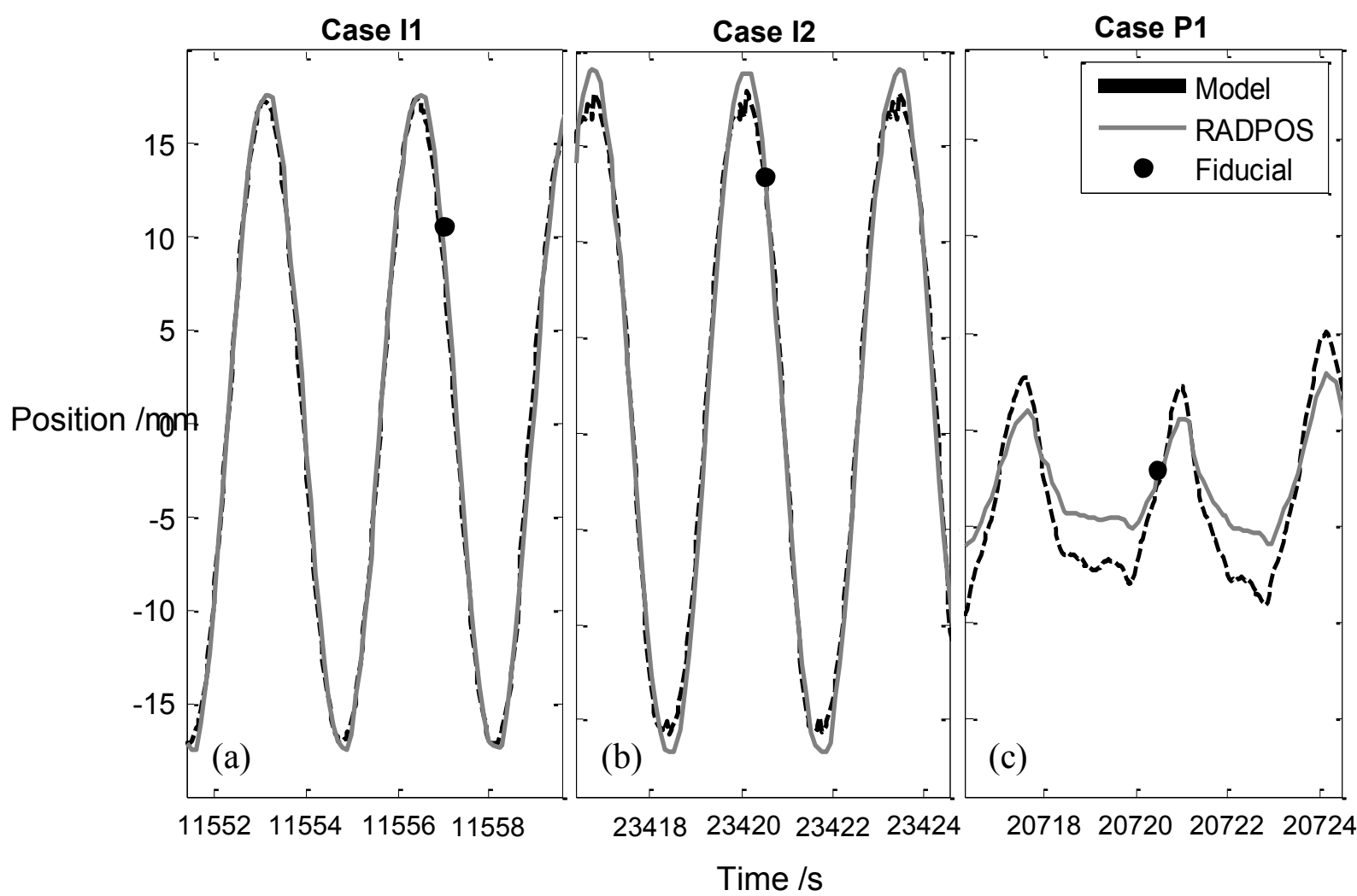

Figure 3.7 Comparison of positional data for the RADPOS position sensor (solid gray curve), Synchrony predictive correlation model (dashed black curve), and x-ray imaged fiducial markers (black dots) after implementation of the coordinate alignment algorithm. The results are shown for treatment cases (a) I1, (b) I2, and (c) P1. The data presented is for the primary direction of motion, as motion along the other directions was small $(<1 \mathrm{~mm}$ peak-to-peak amplitude). The time axes represent the time elapsed since the last program start of the Synchrony system. 
The summary of the results of the positional analysis for the lung experiment are given in Table 3.11, comparing the RADPOS measurements to the Synchrony model and to fiducial measurements. The differences between RADPOS and fiducial position measurements had standard deviations less than $0.5 \mathrm{~mm}$ for all directions and experimental cases. The differences between RADPOS and model position data had standard deviations of $0.82,1.23$, and $2.64 \mathrm{~mm}$ along the primary direction of motion for case I1, I2 and P1, respectively. The standard deviation of the difference was $\leq 0.22 \mathrm{~mm}$ for all comparisons along the $\mathrm{y}$ and $\mathrm{z}$ directions.

Table 3.11 Positional analysis results for the three dynamic treatment cases of the lung experiment, comparing the RADPOS and model positional data. The primary direction of motion for all experimental cases is the $\mathrm{x}$ direction. Comparison 1 was performed in the fiducial time domain, whereas Comparison 2 was performed in either the RADPOS (LED-RADPOS, RADPOS-Model) or Synchrony (LED-MODEL) time domains (see section 2.1.4.2).

Standard Deviation of the Differences ( $\mathrm{mm}$ )

\begin{tabular}{|c|c|c|c|c|c|}
\hline & \multirow{2}{*}{$\begin{array}{l}\text { Position } \\
\text { Datasets }\end{array}$} & \multirow{2}{*}{ Direction } & \multicolumn{3}{|c|}{ Dynamic Cases } \\
\hline & & & $I 1$ & $I 2$ & $P 1$ \\
\hline \multirow{3}{*}{ 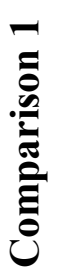 } & & $x$ & 0.32 & 0.42 & 0.28 \\
\hline & RADPOS-Fid & $y$ & 0.13 & 0.19 & 0.12 \\
\hline & & $z$ & 0.17 & 0.18 & 0.13 \\
\hline \multirow{3}{*}{ 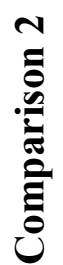 } & & $x$ & 0.82 & 1.23 & 2.64 \\
\hline & RADPOS-Model & $y$ & 0.14 & 0.22 & 0.14 \\
\hline & & $z$ & 0.15 & 0.17 & 0.15 \\
\hline
\end{tabular}




\section{Discussion}

This chapter presents examinations of various experimental aspects, as well as analyses of the experimental results. What follows are discussions of the effects of x-ray imaging on dose measurements, components of dosimetric uncertainty, positional data comparison and uncertainty, dose calculations performed by CyberKnife's treatment planning system, and experiment-specific results analyses. 


\subsection{X-ray Imaging Dose Experiment}

\subsubsection{Film Energy Response}

The x-ray tube peak voltage used during the x-ray imaging dose experiment was $130 \mathrm{kV}$, corresponding to an average photon energy of about three to four times less (i.e., $32 \mathrm{keV}-43 \mathrm{keV})$ [51]. At this low energy photon range ( $40 \mathrm{keV})$, the film's energy dependence was important to consider. Brown et al. [52] have found that EBT3 GafChromic film sensitivity at $35 \mathrm{keV}$ (relative to that of $4 \mathrm{MV} \mathrm{x}$-rays) was within $1 \%$ of unity for an absorbed dose of $50 \mathrm{cGy}$. Alternatively, Villarreal-Barajas and Khan [53] have found that EBT3 sensitivity at $100-150 \mathrm{kVp}$ (relative to that of $1.25 \mathrm{MeV}{ }^{60} \mathrm{Co}$ photons) was approximately $15 \%$ lower for an absorbed dose of $1 \mathrm{~Gy}$. In addition, Massillon-JL et al. [54] have found that EBT3 sensitivity at $50 \mathrm{kVp}$ (relative to $6 \mathrm{MV}$ ) was approximately $11 \%$ lower for an absorbed dose of $50 \mathrm{cGy}$.

Monte Carlo studies have been performed to evaluate the absorbed-dose energy dependence of EBT film relative to the absorbed dose to water and compare simulation results to experimental findings. Sutherland and Rogers [55] found that EBT and EBT2 absorbed-dose energy dependence was energy independent within $0.6 \%$ in the range from $100 \mathrm{keV}$ to $18 \mathrm{MeV}$ but varied by approximately $10 \%$ for EBT below photon energies of $100 \mathrm{keV}$, and that their simulation results did not agree with previous experimental work (which measured the overall energy dependence of film). Simulations performed by Hermida-López et al. [56] were in agreement with the findings of Sutherland and Rogers for EBT and EBT2, while EBT3 results demonstrated reduced absorbed-dose-to-water energy dependence in water (energy independent within $2.3 \%$ in the energy range of 10 $\mathrm{keV}$ and $18 \mathrm{MeV}$ but could be as high as $6 \%$ different from water in solid phantoms at 50 
$\mathrm{keV}$ ). Both groups concluded that the overall energy dependence of EBT film below photon energies of $100 \mathrm{keV}$ is mainly governed by the intrinsic energy dependence, which is affected by the film's active layer chemical reactions and post-irradiation processing [56]. Bekerat et al. [57] have found that EBT3 sensitivity at $40 \mathrm{keV}$ (relative to ${ }^{60} \mathrm{Co}$ ) was approximately $6 \% \pm 4 \%$ lower for an absorbed dose of 2 Gy for the most recent models of EBT3 film which were used for these experiments, and therefore should be sufficiently accurate for the relatively small imaging dose corrections applied in this work.

\subsubsection{MOSFET Sensitivity}

Based on the voltage per $\mathrm{x}$-ray pair values and calibration coefficients determined for the two RADPOS detectors, correction for the apparent dose per x-ray pair value for RADPOS could be estimated using Equations 4.1, 4.2 and 4.3:

$$
\begin{gathered}
(\Delta V / x \text {-ray pair })_{\text {avg }}=\frac{(\Delta V / x \text {-ray pair })_{0669}+(\Delta V / x \text {-ray pair })_{0674}}{2} \\
C F_{\text {avg }}=\frac{C F_{0669}+C F_{0674}}{2} \\
\left(\text { Dose } /_{x \text {-ray pair }}\right)_{R A D P O S}=C F_{\text {avg }} \times\left(\Delta V / /_{\text {-ray pair }}\right)_{\text {avg }}
\end{gathered}
$$

This resulted in a dose correction per x-ray pair of $0.19 \mathrm{cGy}$. Assuming that the film dose calibration applies to $\mathrm{kV}$ photons, this RADOS dose correction value was approximately four times larger than the measured film value, which yielded a dose correction per x-ray pair of $0.04747 \mathrm{cGy}$.

This apparent over-response has been observed for both measured data and Monte Carlo simulations of the MOSFET in a $6 \mathrm{MV}$ photon beam. Figure 4.1 illustrates the 
findings of Wang et al. [58] ('This study') and others, where MOSFET sensitivity is near unity at higher photon energies but increases significantly at lower photon energies. It can be seen that at photon energies around $40 \mathrm{keV}$, MOSFET sensitivity rises to about four times its value at megavoltage and high (i.e., several hundred) kilovoltage energies.

This MOSFET over-response was also observed experimentally for the results of the x-ray imaging dose experiment (see section 3.1). A 3-to-4-fold rise in the effect of the imaging $\mathrm{x}$-rays for the RADPOS relative to film was observed for both the isocentric (2$3 \%$ for RADPOS versus $0.7-0.8 \%$ for film) and non-isocentric (5\% for RADPOS versus $1.5 \%$ for film) cases of the lung experiment.

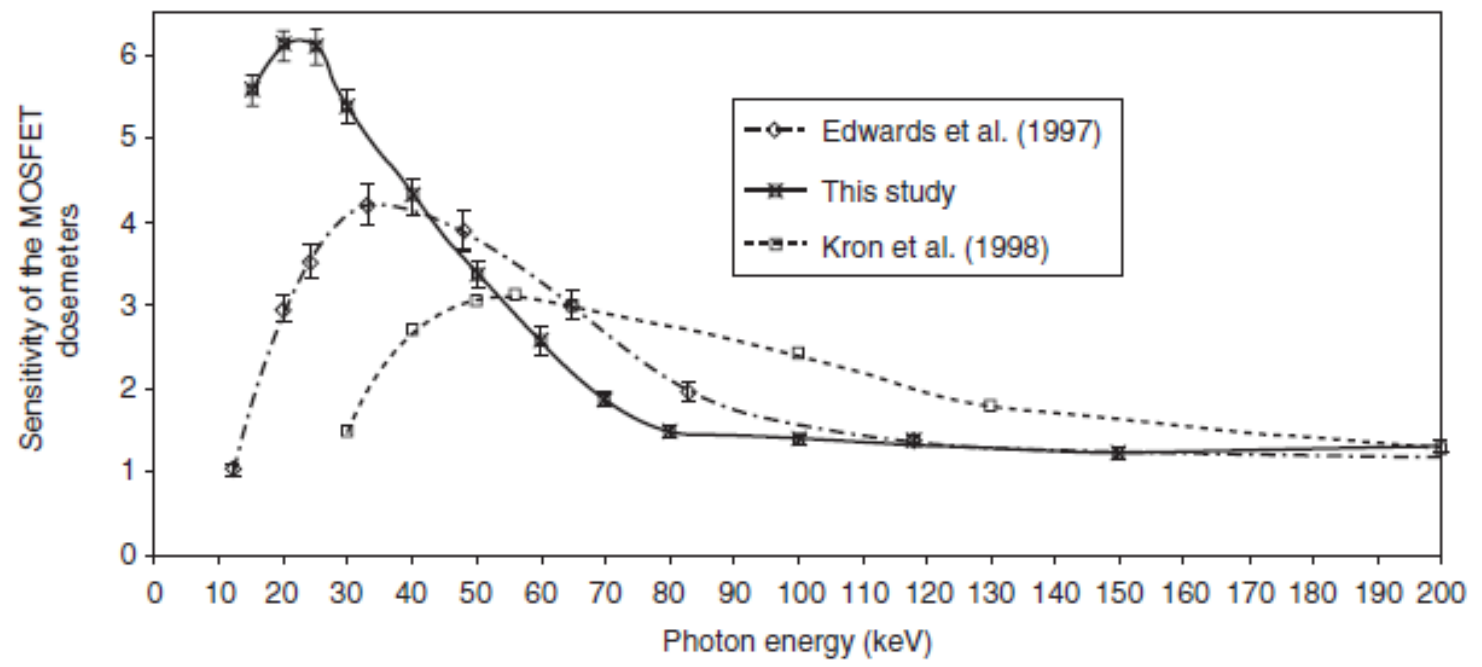

Figure 4.1 Response of MOSFET dosimeter as a function of photon energy. A significant overresponse is observed at lower photon energies. Obtained with permission from Wang et al., 2005 [58].

Lastly, using the correction per x-ray pair values determined for film dose and RADPOS voltage, a MOSFET calibration coefficient could be estimated for the $\mathrm{kV}$ x-ray energy range, as show in equation 4.4:

$$
C F_{k V}=\frac{(D / x \text {-ray pair })}{(\Delta V / x \text {-ray pair })_{\text {avg }}}
$$

This resulted in a MOSFET $\mathrm{kV}$ x-ray energy calibration coefficient of $0.10 \mathrm{cGy} / \mathrm{mV}$. 


\subsubsection{Estimate versus Measurement of Dose Per X-ray Pair}

The ME scaling factor determined in section 3.1.3, which was a ratio of the measured to estimated film dose per x-ray pair value for the geometry of the lung experiment, was determined to be 2.14. This meant that the estimated value accounted for only about half of the total measured dose absorbed by the film within the lung insert. This discrepancy was largely due to the relatively low energy $\mathrm{x}$-ray photons $(\sim 40 \mathrm{keV})$ produced by the x-ray tube. At such low energy, a large fraction of the total attenuation was attributable to photon scatter. Therefore, scattered photons could not be readily removed from the incident beam and could contribute more to local dose absorption. The estimate of the dose per x-ray pair (see section 2.2.2) only took the linear attenuation of photons into account, and did not consider additional dose due to scatter.

In addition, at this low photon energy range, the percent of photoelectric interactions (i.e., $\tau / \mu$ ), as well as the percent of energy transferred due to photoelectric interactions (i.e., ${ }^{{ }_{t r}} / \mu_{t r}$ ), was relatively high [51]. Together with the fact photoelectrons set in motion by low energy photons do not tend to travel in a forward-directed manner when ejected, the primary effect of photoelectric collisions in the case of low energy photons was local energy absorption. Again, this was not taken into account for the estimate of the dose per x-ray pair.

Also, the static and dynamic phantom cases of the breast experiment involved approximately the same number of imaging x-rays on average during delivery (i.e., 32 and 33 respectively), as both cases involved the same isocentric treatment plan. The estimated dose difference due to x-rays accounted for approximately $0.3 \%$ of the total uncorrected film dose. Based on the previous discussion regarding MOSFET over- 
response, the estimated voltage difference due to x-rays is expected to account for approximately $1 \%$ of the total uncorrected RADPOS voltage. Although these values are estimates, they indicate that the effect of x-ray imaging dose during the breast experiment may be comparable to the lung experiment and that as future work, an experimental investigation could be performed to determine more accurate estimates of the imaging dose.

\subsection{Dose Uncertainty}

\subsubsection{Beam Delivery}

From Synchrony's ModelPoints.log file, an estimate of the overall beam delivery error was computed for the case of a single treatment beam and for a typical isocentric plan delivered using a $35 \mathrm{~mm}$ cone with the RADPOS detector at beam intersection point. The results were then generalized to the case of multiple beams, as in a clinical treatment. Using the Synchrony correlation error data, the radial error $\left(\sigma_{\mathrm{r}}\right)$ was calculated by summing the errors along the $\mathrm{x}, \mathrm{y}, \mathrm{z}$ directions in quadrature. A confidence interval of $95 \%$ was selected for beam delivery errors falling within $2 \sigma_{\mathrm{r}}$, resulting in an estimated dosimetric uncertainty of $0.5 \%$ (based on inverse-square law corrections and radial profiles measured at various depths for the $35 \mathrm{~mm}$ collimator at the time of commissioning).

Beam delivery was also affected by the stability of CyberKnife's MU (monitor unit) chamber. For a single beam treatment, this lead to a dosimetric uncertainty of about $0.25 \%(1 \sigma)$. A typical CyberKnife isocentric treatment plan consisted of approximately 
25 beams, and so the dosimetric uncertainty due to the radial error and MU stability was scaled by $1 / \sqrt{N}$ (i.e., $1 / \sqrt{25}$ ) to $0.1 \%$ and $0.05 \%$, respectively.

Non-isocentric treatment delivery involves non-coplanar beams which are not restricted to intersect at a common point (i.e., the isocenter) within the target, as is the case with isocentric delivery. This type of treatment delivery gives CyberKnife the versatility to effectively irradiate an irregularly shaped target while avoiding healthy tissue or OAR, as illustrated in Figure 4.2. But having the treatment beams not intersect at a common point leads to a higher level of uncertainty due to the resulting complex distribution of high dose ("hot") and low dose ("cold") regions in the vicinity of the target. The location of these hot and cold regions is difficult to predict and correct for because of the complicated spatial arrangement of treatment beams. In addition, the RADPOS detector was often in a large dose gradient for many beams during the nonisocentric deliveries of the lung experiment (cases P0 and P1). Factors contributing to this included the detector's size relative to the small beams $(15 \mathrm{~mm}$ and $30 \mathrm{~mm}$ diameter for this plan) of the CyberKnife and the motion of the detector within the moving Quasar phantom during treatment delivery.

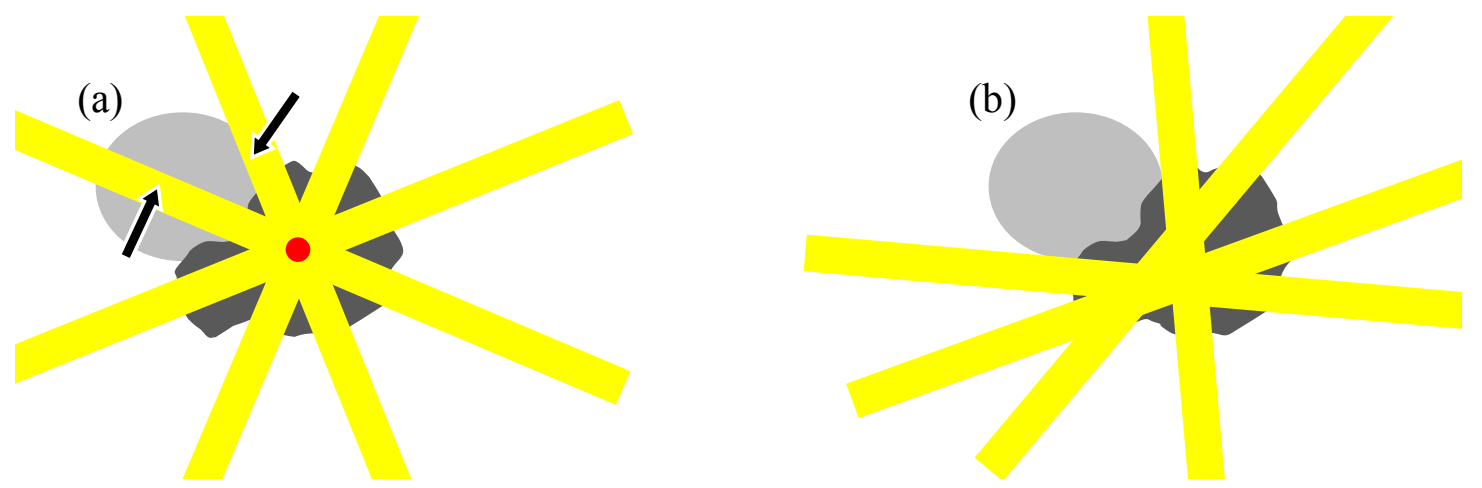

Figure 4.2 Illustration of the main differences between (a) isocentric and (b) non-isocentric treatment deliveries on the CyberKnife system. In both cases, the OAR, tumor, and treatment beams are represented by the light gray oval, dark gray irregularly-shaped object, and transparent lines, respectively. In the case of isocentric delivery, the beams intersecting at a common point (isocenter, red dot) pass through the OAR (indicated by the black arrows). For this fictitious example, the OAR is completely spared of entrance and exit dose in the case of non-isocentric delivery. 


\subsubsection{Beam Dosimetry}

An estimate of the dosimetric uncertainty due to beam dosimetry and crosscalibration was computed based on the addendum to the AAPM's TG-51 report [59]. The cross-calibrations that were performed after all of the experiments used the same ion chamber with the RADPOS detector or film in the same position as the chamber. As a result, many of the components of uncertainty that are concerned with measurement and influence quantities are cancelled out and would not be detectable in a dosimetric comparison between film and RADPOS detectors.

The components of uncertainty that are concerned with measurement consist of parameters such as SSD, depth, field-size, as well as pressure and temperature corrections. Additionally, the components of uncertainty that are concerned with ion chamber influence quantities include leakage current and corrections factors for polarity, recombination and electrometer effects. Also, the components of uncertainty based on calibration data for the ion chamber used for cross calibration, such as the calibration coefficient $\left(\mathrm{N}_{\mathrm{D}, \mathrm{w}}\right)$ and beam quality correction factor $\left(\mathrm{k}_{\mathrm{Q}}\right)$, remain unchanged for RADPOS and film. Lastly, due to the fact that CyberKnife cannot create the standard reference $10 \times 10 \mathrm{~cm}^{2}$ field-size, an error is introduced by the process determined to obtain the beam quality correction factor. This error has been independently determined to be less than $0.3 \%[60,61]$. 


\subsubsection{Detector Measurements}

\subsubsection{RADPOS}

The average standard deviation for threshold voltage difference measurements of the RADPOS detectors used for the various experiments was approximately $\pm 1 \%$. This reproducibility was consistent with the findings of other research groups [27, 62-67], which found that MOSFET measurement variability was generally within $\pm 2-3 \%$ for most cases.

\subsubsection{Film}

The dose measurement-to-measurement reproducibility of the EBT3 GafChromic film used for the various experiments was approximately $\pm 1.3 \%$. This estimate was based on comparing similar region-of-interest intensity values (accomplished with ImageJ) for fourteen known reference dose film pieces from the lung experiment. The standard deviation of the fourteen intensity values gave rise to the $1.3 \%$ reproducibility result. This reproducibility was consistent with the findings of other research groups, where film reproducibility has been shown to be within $\pm 2 \%$ in the case of using a single film [37, 41, 68]. This total uncertainty for GafChromic film dosimetry has been estimated to be $1.44 \%$ when using two films and $1.40 \%$ when using three films [37].

\subsubsection{Phantom Material}

It has been shown that the dose measured by certified grade Solid Water, such as the breast phantom and lung tumor phantom used for the experiments, differs by $0.7 \%$ from that in liquid water [69]. Of this, approximately $0.3 \%$ is due to the dosimetric 
difference between dose-to-medium versus dose-to-water calculations, and approximately $0.6 \%$ is due to variability in manufacturing [69]. The dosimetric difference between doseto-medium versus dose-to-water calculations arises from what Seuntjens et al. define as the solid phantom-to-water dose conversion factor, $k_{s, w}^{Q}$, which represents the water equivalence of a phantom and is generally close to 1 (a $k_{s, w}^{Q}$ of exactly 1 indicates perfect water equivalence). $k_{s, w}^{Q}$ depends on conditions such as the depth and field size at which the dose is measured within the solid phantom, and is therefore subject to change with irradiation conditions [69].

\subsubsection{Summary}

Table 4.1 gives a summary of the various sources of dosimetric uncertainty (under isocentric treatment delivery conditions) encountered for the various experiments performed, along with the associated uncertainty evaluation type (as described by the International Standards Organization [70]). Type A evaluations of uncertainty are based on the statistical analysis of a series of observations (e.g., computing the standard deviation of the mean), while Type B evaluations of uncertainty are based on scientific judgement using relevent and available information (e.g., determining the standard uncertainty from a calibration certificate) [70]. 
Table 4.1 Summary of the various sources of dosimetric uncertainty, along with the associated uncertainty evaluation type.

\begin{tabular}{|c|c|c|c|c|}
\hline & & & $\begin{array}{l}\text { Uncertainty } \\
\text { Magnitude }\end{array}$ & $\begin{array}{c}\text { Uncertainty } \\
\text { Evaluation } \\
\text { Type } \\
\end{array}$ \\
\hline \multirow{4}{*}{$\begin{array}{c}\text { Beam } \\
\text { Delivery }\end{array}$} & \multirow{2}{*}{$\begin{array}{l}\text { Single beam } \\
\text { (with detector on } \\
\text { central axis) }\end{array}$} & Radial error & $0.5 \%$ & A \\
\hline & & MU chamber stability & $0.25 \%$ & A \\
\hline & \multirow{2}{*}{$\begin{array}{c}\text { Whole plan } \\
\text { (Isocentric Planning, } \\
20 \text { beams) }\end{array}$} & Radial error & $0.1 \%$ & A \\
\hline & & MU chamber & $0.06 \%$ & A \\
\hline \multirow{3}{*}{$\begin{array}{c}\text { Beam } \\
\text { dosimetry }\end{array}$} & \multirow{3}{*}{ Calibrations } & User dependent & $0.3 \%$ & $\mathrm{~A}+\mathrm{B}$ \\
\hline & & Calibration data & $0.85 \%$ & B \\
\hline & & $\mathrm{k}_{\mathrm{Q}}$ approximation & $0.3 \%$ & B \\
\hline \multirow{10}{*}{$\begin{array}{c}\text { Detector } \\
\text { measurement }\end{array}$} & \multirow{5}{*}{ Film } & Beam delivery & $0.1 \%$ & A \\
\hline & & Beam dosimetry & $0.95 \%$ & $\mathrm{~A}+\mathrm{B}$ \\
\hline & & Phantom material & $0.7 \%$ & $\mathrm{~B}$ \\
\hline & & $\begin{array}{l}\text { Single film } \\
\text { reproducibility }\end{array}$ & $2.0 \%$ & $\mathrm{~A}+\mathrm{B}$ \\
\hline & & Film total & $2.3 \%$ & $\mathrm{~A}+\mathrm{B}$ \\
\hline & \multirow{5}{*}{ RADPOS } & Beam delivery & $0.1 \%$ & A \\
\hline & & Beam dosimetry & $0.95 \%$ & $\mathrm{~A}+\mathrm{B}$ \\
\hline & & Phantom material & $0.7 \%$ & B \\
\hline & & RADPOS reproducibility & $1.0 \%$ & $A+B$ \\
\hline & & RADPOS total & $1.6 \%$ & $\mathrm{~A}+\mathrm{B}$ \\
\hline
\end{tabular}




\subsection{Position Measurements}

\subsubsection{Position Uncertainty}

The uncertainty on RADPOS, LED and fiducial positions was taken into account during positional data analysis involving the coordinate alignment algorithm.

There were a few aspects of the RADPOS, LED and fiducial positional data which had a bearing on the uncertainty. First, the LED and fiducial position data were recorded to two decimal places (LEDs) or greater (fiducials), meaning that measurements had measurement precision of $0.005 \mathrm{~mm}$. However, RADPOS position measurements could only be recorded to one decimal place, resulting in a resolution of $0.05 \mathrm{~mm}$.

Second, the LED sampling rate was fixed, while the RAPDOS sampling rate was set to be as frequent as possible. Still, the LED marker positions were sampled approximately 2.5 times as frequently as RADPOS positions were. The relatively low RADPOS position sampling frequency introduced an uncertainty with respect to time (phase-shift) when compared to the LED marker signal. In addition, an inherent temporal uncertainty arose during the user's manual selection of a common point on the two motion signals when running the coordinate alignment algorithm.

To account for these two temporal uncertainties and translate them into a positional error, the time uncertainties of the LED and RADPOS measurements were summed in quadrature. In both cases, these uncertainties were taken as half of the sampling rate for LEDs (40 ms) and RADPOS (100 ms), leading to approximately $20 \mathrm{~ms}$

for LEDs and $50 \mathrm{~ms}$ for RADPOS. This resulted in an overall uncertainty in time, $\sigma_{\mathrm{t}}$, of approximately $54 \mathrm{~ms}$. The uncertainty in position was obtained by adding and subtracting $\sigma_{t}$ from the point of signal alignment. This yielded an upper and lower bound on the 
standard deviation of the difference values and lead to positional uncertainties of \pm 0.02 $\mathrm{mm}, \pm 0.04 \mathrm{~mm}$, and $\pm 0.19 \mathrm{~mm}$ along the $\mathrm{x}$ (superior/inferior), $\mathrm{y}$ (left/right) and $\mathrm{z}$ (anterior/posterior) directions, respectively. This analysis was done using data from the breast experiment, where the $\mathrm{z}$ direction was the primary direction of motion. In the case of the lung experiment where the $\mathrm{x}$ direction was the primary direction of motion, the positional uncertainties were distributed accordingly: $\pm 0.19 \mathrm{~mm}$ along the $\mathrm{x}$ direction, and $\pm \sim 0.03 \mathrm{~mm}$ along the $\mathrm{y}$ and $\mathrm{z}$ directions.

A component of measured movement in a direction(s) which were not expected to have any motion present (e.g., $\mathrm{x}$ and $\mathrm{y}$ directions in breast experiment, $\mathrm{y}$ and $\mathrm{z}$ directions in lung experiment) was attributable to noise. To quantify the effect of noise, the standard deviation was computed for position measurements not along the primary direction of motion for the different position tracking modalities. It was found that the effects of noise did not exceed approximately $0.10 \mathrm{~mm}\left(1 \sigma_{\mathrm{S}}\right)$ for the Synchrony system (i.e., LEDs and fiducials) alone, but use of the RADPOS increased the overall effects of noise to approximately $0.30 \mathrm{~mm}\left(1 \sigma_{\mathrm{S}+\mathrm{R}}\right)$.

It is important to note that measured movement along the non-primary direction of motion may have been real motion along those axes if the phantom was not aligned perfectly with the treatment room (i.e., fiducial) coordinate system. Although care was taken during setups to ensure that this was not the case, the setup error, which was likely on the order of 1-2 mm (i.e., displacement between setup of apparatus on the CyberKnife treatment couch vs planning CT scan), was mitigated via couch corrections during pretreatment CyberKnife x-ray imaging. 
Table 4.2 summarizes the various contributions to the overall positional uncertainty for the general case of motion taking place primarily in one direction.

Table 4.2 Summary of the various contributions to the overall positional uncertainty for the general case of motion along a single direction. The uncertainties along the primary and non-primary directions of motion are given, along with how the uncertainties change with and without RADPOS.

\begin{tabular}{cccccc}
\hline \multirow{2}{*}{ Direction } & Position Tracking & \multicolumn{3}{c}{ Uncertainty (mm) } \\
& Modalities & Resolution & Temporal & Noise & Total \\
\hline $\begin{array}{c}\text { Primary Direction } \\
\text { of Motion }\end{array}$ & Synchrony & N/A & $\sim 0.2$ & 0.1 & $\mathbf{0 . 2 2}$ \\
\hline \multirow{2}{*}{ Other Directions } & Synchrony and RADPOS & 0.05 & $\sim 0.2$ & 0.3 & $\mathbf{0 . 3 6}$ \\
& Synchrony and RADPOS & 0.05 & $\sim 0.03$ & 0.3 & $\mathbf{0 . 3 1}$ \\
\hline
\end{tabular}

\subsubsection{Position Data Comparison}

All data comparisons between position tracking modalities involved computing the standard deviation of the differences. This was selected as the comparative metric in order to give a reliable measure of how different the motion signal distributions were and because the least-squares-based coordinate alignment algorithm minimized the average differences.

Throughout the various experiments, motion measurements were made using different position tracking modalities: RADPOS, LEDs, and fiducials. The goal was then to compare the measurements made by these modalities and evaluate how similar or different they were. Since the LED sampling rate was higher than that of the RADPOS, more data points were generated for the same interval of time. Therefore, the LED data points were interpolated to the RADPOS time points during positional comparison.

The differences of the $\mathrm{x}, \mathrm{y}, \mathrm{z}$ coordinates were taken and the standard deviation was calculated, which yielded a spread of the differences between the RADPOS and 
(interpolated) LED position data. A small spread was indicative of similar datasets, which in turn demonstrated agreement of the different position measurement modalities.

Comparison 1 (performed in the fiducial time domain, see section 2.1.4.2) involved a single interpolation step (LED or RADPOS points into fiducial time domain) before the coordinate alignment algorithm was used. Comparison 2 (performed in the RADPOS or Synchrony time domains, see section 2.1.4.2) generally involved two interpolation steps (LED or RADPOS or model data points into fiducial time domain, and LED or model into RADPOS time domain). The data was examined in this way in order to perform comparisons based on the fewest amount of intermediate interpolation steps as possible.

For Comparison 1, the average difference between fiducial and LED measurements or fiducial and RADPOS measurements was very small $(<0.01 \mathrm{~mm})$ for all directions because of the least-squares-based minimization of the coordinate alignment algorithm. The results were similar for Comparison 2, where the average difference between LED, RADPOS and model measurements (breast experiment) or just RADPOS and model measurements (lung experiment) was $<0.05 \mathrm{~mm}$ for all directions. Larger average differences were found for Comparison $2(<0.05 \mathrm{~mm})$ than for Comparison 1 $(<0.01 \mathrm{~mm})$ because any errors associated with applying the same translation vector to an interpolated (i.e., relatively small) dataset would be increased when applying the same translation vector to a non-interpolated (i.e., original, larger) dataset (see step 6 of section 2.1.4.2). Therefore, using the standard deviation of the differences, rather than just the average differences, was the best comparative metric for this work. 


\subsection{Experiment-Specific Discussions}

\subsubsection{Breast Experiment}

\subsubsection{Dose Results}

The dose values calculated by the ray tracing and Monte Carlo algorithms of the treatment planning system had negligible difference and were within $1 \%$ of each other, which was within the overall uncertainty of Monte Carlo calculations. This was because both calculations were done for the primarily homogeneous, unit-density material of the Solid Water breast phantom. All dose value comparisons done for the static phantom case resulted in differences which were within $1 \sigma$ of experimental uncertainties. For the dynamic phantom case, all dose value comparisons resulted in differences which were within $2 \sigma$ of experimental uncertainties. These results demonstrate that the effects of target motion during radiotherapy (even simple, sinusoidal motion along one direction) are not insignificant, and effort should be put towards minimizing the errors and uncertainties inherent to target motion.

The Synchrony model and RADPOS position measurements were not in perfect agreement (differences were within $2 \sigma$ of experimental uncertainties) for the dynamic phantom cases. This is apparent from the dosimetric results, where the RADPOS and film dose values were in excellent agreement (within $0.1 \%$ of one another) for the static case but differed more (within 3.3\% of one another) for the dynamic case. Since isocentric treatment plans (such as the one used for the breast experiment) are less sensitive to errors near the point of intersection of the beams (i.e., isocenter) for larger collimator sizes (e.g., $35 \mathrm{~mm}$ ), the $>1 \sigma$ deviation between RADPOS and model position data is likely due to RADPOS or film dosimetric uncertainties rather than model quality. 


\subsubsection{Position Results}

Comparison 1 (performed in the fiducial time domain) for the LED and fiducial position measurements, as well as Comparison 2 (performed in the Synchrony time domain) for the LED and model position data, yielded differences along the $\mathrm{x}$ and $\mathrm{z}$ directions (non-primary direction of motion and primary direction of motion, respectively) which were within $1 \sigma$ of positional uncertainties (for the case of Synchronyonly measurements, see Table 4.2). The significance of Comparison 1 was two-fold: it provided a measure of the accuracy of CyberKnife's imaging components, and it quantified the effectiveness of the coordinate alignment algorithm, where the LED position measurements were transformed to the fiducial frame of reference. In future studies, this comparison could be used as a benchmark for how successfully the algorithm has worked for more complex datasets. Also, Comparison 2 benchmarked the agreement between the LEDs and the Synchrony model in the simple case where the motion of the LEDs and fiducials were in the same direction, in phase, and moving with sinusoidal motion.

Similarly, Comparison 1 for the RADPOS and fiducial position measurements, as well as Comparison 2 for the RADPOS and model position data, yielded differences which were within $1 \sigma$ (x direction) and within $3 \sigma$ (y and $\mathrm{z}$ directions) of positional uncertainties. Comparison 1 quantified the effectiveness of transforming the RADPOS position measurements to the fiducial frame of reference. Both Comparison 1 and Comparison 2 were used as benchmarks for their corresponding analyses in the lung experiment, where RADPOS position measurements were compared to fiducial and 
model data along the superior-inferior direction for a larger amplitude motion of $36 \mathrm{~mm}$ peak-to-peak.

The LED and RADPOS position measurements were in good agreement to approximately $0.5 \mathrm{~mm}$ and less, with differences along all directions being within $2 \sigma$ of positional uncertainties. All of the comparisons (which involved the LED and RADPOS position data) yielded the largest differences along the y direction even though motion in the fiducial frame of reference was predominantly along the $\mathrm{z}$ direction. The reason for this is when the LED and RADPOS position measurements were transformed into the fiducial frame of reference, the resulting y-direction data for both system was non-zero, with a peak-to-peak amplitude of about $2 \mathrm{~mm}$. The cause of this coordinate transformation shortcoming was not clear and was not present for the lung experiment (see Table 3.11). A possible explanation is that any motion recorded along the nonprimary direction of motion for the RADPOS and LED systems during the breast experiment, where the peak-to-peak amplitude of the breast phantom was $10 \mathrm{~mm}$, was a higher fraction of the total overall motion than in the case of the lung experiment, where the peak-to-peak amplitude of the lung insert was $36 \mathrm{~mm}$. Because of this, the coordinate alignment algorithm may not have performed the transformations into the fiducial coordinate system as effectively.

\subsubsection{Lung Experiment}

\subsubsection{Dose Results}

Dose calculations were performed on the max inferior, mid respiration, and max superior phases of the lung insert's motion within the Quasar. This was done in order to 
obtain an estimate on how dose delivery changed with respect to target motion during a typical CyberKnife treatment. In addition to mid respiration, the positional extremes of the maximum inferior and superior lung insert were selected so that the trajectory of treatment beams passing through the Quasar would differ the most (see Figure 2.26) in terms of how much material they would be passing through on their way to the target (i.e., Solid Water ball).

In all cases, the maximum inferior and superior dose values were greater than the mid respiration values. This is because during the mid respiration phase, the target is at the center of the Quasar acrylic body. Therefore, most (if not all) of the CyberKnife treatment beams pass through the acrylic material and are attenuated. During the maximum inferior or superior phases, the target moves closer to the outside of the acrylic body. As a result, some treatment beams may pass through less material and be attenuated to a smaller degree, or avoid the acrylic body entirely and strike the target unimpeded. The fact that the maximum superior dose values are $2-3 \%$ higher than the mid respiration values while the maximum inferior dose values are only $1-2 \%$ higher than the mid respiration values means that the distribution of CyberKnife treatment beams was not symmetric (at least not in the superior/inferior direction) around the target, and a higher net dose was being delivered to the superior end than to the inferior end. This is consistent with the fact that the density of node positions is higher superiorly than inferiorly and would be affected less by the inverse-square law.

The TPS dose was computed at two coronal positions: the RADPOS plane and the film plane (see Figure 3.4). The film was positioned just on top of the RADPOS detector within the lung insert, and the variation of the dose distribution from the film to the 
RADPOS was not insignificant because of the differences in density of the two dose detectors. The density of GafChromic film was very similar to that of water, while the density of RADPOS' microMOSFET dosimeter was much higher $\left(\sim 2.4 \mathrm{~g} / \mathrm{cm}^{3}\right)$. The film plane TPS dose values were larger than the RADPOS plane values for all experimental cases and respiratory phases. This was likely due to the backscatter of treatment beam photons from the high density RADPOS detector back up to the GafChromic film, leading to an increased overall film dose in that region. This was generally consistent with measured film dose, where film values were approximately $1.2 \%$ higher on average than RADPOS dose values.

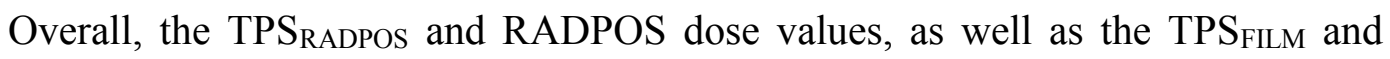
film dose values, were in good agreement, with most differences falling within $2 \sigma$ of experimental uncertainties. In addition, the RADPOS and film dose values agreed to within $2 \sigma$ for all experimental cases.

\subsubsection{Position Results}

Comparison 1 for the RADPOS and fiducial position measurements yielded differences which were approximately within $1 \sigma$ of positional uncertainties along all directions for all experimental cases. Additionally, this comparison was consistent with the corresponding comparison (i.e., benchmark) from the breast experiment.

Comparison 2 for the RADPOS and model position data yielded differences which were within $1 \sigma$ of positional uncertainties along the y and $\mathrm{z}$ directions for all experimental cases. The differences along the $\mathrm{x}$ direction were large (greater than $2 \sigma$ ) for all cases, and significantly exceeded the benchmark comparison from the breast 
experiment. Other studies [71, 72] involving CyberKnife treatments of lung patients have found correlation errors of the Synchrony model (i.e., difference between where the model thinks the target is and where the target actually is) to be as large as 2.5 to $3.5 \mathrm{~mm}$, which is consistent with the Comparison 2 results of RADPOS versus Synchrony model data.

Although the results showed that while both a phase difference between the Quasars (cases I2 and P1) and complex, patient-like motion (case P1) reduced the quality of the correlation model, it was the complexity of the motion which had a more significant effect on the RADPOS-model agreement than did the phase difference. This is because the difference between the RADPOS and model position data increased by approximately $0.4 \mathrm{~mm}$ when changing from simple, in-phase motion (I1) to simple, outof-phase motion (I2). However, the difference increased by approximately $1.4 \mathrm{~mm}$ when changing from simple, out-of-phase motion (I2) to complex, out-of-phase motion (P1).

Therefore, it seemed that the quality of the correlation model was highly dependent on the motion reproducibility and patterns of the fiducials and LEDs, and not as much on a phase difference or time delay (hysteresis) between them. The Synchrony system is equipped to deal with hysteresis, as it implements a linear, quadratic, or constrained fourth order polynomial fit for the Synchrony model depending on the amount of time delay between the measured motion signals. 


\section{Conclusions}

This chapter gives a summary of the results obtained for the breast and lung

experiments. In addition, the clinical potential of the RADPOS system in both quality assurance tests and patient treatments is described. 


\subsection{Summary of Results}

\subsubsection{Breast Experiment}

RADPOS position measurements closely matched LED marker positions, with less than $0.5 \mathrm{~mm}$ standard deviation between them on average over all directions. In addition, RADPOS, film, and TPS dose values were in good agreement, with all comparisons falling within $2 \sigma$ of experimental uncertainty. Implementation of the coordinate alignment algorithm successfully allowed for the consistent comparison of position measurement datasets within a common coordinate system. In conclusion, RADPOS is very accurate and precise when it comes to functioning as a dose and position measurement quality assurance tool for CyberKnife.

\subsubsection{Lung Experiment}

RADPOS position measurements agreed with fiducial position readings given by the x-ray tracking system to the sub-millimetre level. In addition, the difference between the RADPOS and CyberKnife's correlation model, which was $2.64 \mathrm{~mm}$ for the most complex motion case, was consistent with experimental findings of other groups. The dosimetric results between the RADPOS, film, and TPS were in good agreement, with most differences falling within $2 \sigma$ of experimental uncertainties. In conclusion, RADPOS can be used to evaluate how CyberKnife treatment delivery is affected by complex phantom motion and respiratory hysteresis effects. 


\subsection{Clinical Potential of RADPOS}

As a quality assurance tool, RADPOS provides point dose and position measurements simultaneously and instantly. In addition to the quality assurance opportunities for RADPOS outlined in section 1.5, RADPOS could also be useful for performing beam shape and beam symmetry tests, as well as End-to-End tests, as part of the monthly CyberKnife QA protocol [28].

RADPOS has much potential when it comes to its clinical applicability, and this work was a necessary first step before RADPOS could safely and reliably be used for in vivo dose and position measurements for patient treatments. More specifically, RADPOS could be used for intracorporeal dose and position measurements by inserting the probe into a Foley catheter (for prostate applications) or a tracheal tube (for bronchial applications). Alternatively, extracorporeal dose and position measurements for breast and lung applications could be achieved by attaching RADPOS to the surface of the patient. The RADPOS electromagnetic positioning sensor offers an advantage over the LED optical tracking used by the Synchrony system in that position tracking of the LED optical markers can only be accomplished if they are clearly visible by the stereoscopic

camera. In situations where this is not possible (e.g., patient comfort is compromised), RADPOS could be used to obtain those position measurements.

In summary, RADPOS' ability to perform simultaneous dose and position measurements makes it a powerful option for use as a clinical quality assurance tool for the CyberKnife radiosurgery system. 


\section{References}

1. Canadian Cancer Society's Advisory Committee on Cancer Statistics. Canadian Cancer Statistics 2014. Toronto, ON: Canadian Cancer Society, 2014.

2. Physician Characteristics and Distribution in the U.S. Statistics About Radiation Therapy. 2010; Available from:

http://www.rtanswers.org/statistics/aboutradiationtherapy/.

3. Brown, J.M., D.J. Carlson, and D. Brenner, The tumor radiobiology of SRS and

SBRT: are more than the 5 Rs involved? Int J Radiat Oncol Biol Phys, 2014. 88: p. 254-262.

4. De Groote, A., M. Wantier, G. Cheron, M. Estenne, and M. Paiva, Chest wall motion during tidal breathing. J Appl Physiol, 1997. 83: p. 1531-1537.

5. Seppenwoolde, Y., H. Shirato, K. Kitamura, S. Shimizu, M. van Herk, J.V. Lebesque, and K. Miyasaka, Precise and real-time measurement of 3D tumor motion in lung due to breathing and heartbeat, measured during radiotherapy. Int J Radiat Oncol Biol Phys, 2002. 53: p. 822-834.

6. Bissonnette, J., K.N. Franks, T.G. Purdie, D.J. Moseley, J. Sonke, D.A. Jaffray, L.A. Dawson, and A. Bezjak, Quantifying interfraction and intrafraction tumor motion in lung stereotactic body radiotherapy using respiration-correlated cone beam computed tomography. Int J Radiat Oncol Biol Phys, 2009. 75: p. 688-695.

7. Weiss, E., K. Wijesooriya, S. Vaughn Dill, and P.J. Keall, Tumor and normal tissue motion in the thorax during respiration: analysis of volumetric and positional variations using 4D CT. Int J Radiat Oncol Biol Phys, 2007. 67: p. 296-307.

8. Ekberg, L., O. Holmberg, L. Wittgren, G. Bjelkengren, and T. Landberg, What margins should be added to the clinical target volume in radiotherapy treatment planning for lung cancer? Radiotherapy and Oncology, 1998. 48: p. 71-77.

9. Chen, G.T.Y., J.H. Kung, and K.P. Beaudette, Artifacts in computed tomography scanning of moving objects. Seminars in Radiation Oncology, 2004. 14: p. 19-26.

10. Balter, J.M., R.K. Ten Haken, T.S. Lawrence, K.L. Lam, and J.M. Robertson, Uncertainties in CT-based radiation therapy treatment planning associated with patient breathing. Int J Radiat Oncol Biol Phys, 1996. 36: p. 167-174.

11. Ross, C.S., D.H. Hussey, E.C. Pennington, W. Stanford, and J.F. Doornbos, Analysis of movement of intrathoracic neoplasms using ultrafast computerized tomography. Int J Radiat Oncol Biol Phys, 1990. 18: p. 671-677.

12. Chaudhari, S.R., S.M. Goddu, D. Rangaraj, O.L. Pechenaya, W. Lu, E. Kintzel, K. Malinowski, P.J. Parikh, J.D. Bradley, and D.A. Low, Dosimetric variances anticipated from breathing-induced tumor motion during tomotherapy treatment delivery. Phys Med Biol, 2009. 54: p. 2541-2555.

13. Engelsman, M., E. Damen, K. De Jaeger, K. Van Ingen, and B. Mijnheer, The effect of breathing and set-up errors on the cumulative dose to a lung tumor. Radiotherapy and Oncology, 2001. 60: p. 95-105. 
14. Lujan, A.E., J.M. Balter, and R.K. Ten Haken, A method for incorporating organ motion due to breathing into $3 D$ dose calculations in the liver: sensitivity to variations in motion. Med Phys, 2003. 30: p. 2643-2649.

15. Keall, P.J., G.S. Mageras, J.M. Balter, R.S. Emery, K.M. Forster, S.B. Jiang, J.M. Kapatoes, H.D. Kubo, D.A. Low, M.J. Murphy, B.R. Murray, C.R. Ramsay, M.B. van Herk, S.S. Vedam, J.W. Wong, and E. Yorke, The management of respiratory motion in radiation oncology report of AAPM Task Group 76. Med Phys, 2006. 33: p. 3874-3900.

16. Keall, P.J., V.R. Kini, S.S. Vedam, and R. Mohan, Potential radiotherapy improvements with respiratory gating. Australas Phys Eng Sci Med, 2002. 25: p. 1-6.

17. Keall, P.J., V.R. Kini, S.S. Vedam, and R. Mohan, Motion adaptive x-ray therapy: a feasibility study. Phys Med Biol, 2001. 46: p. 1-10.

18. International Commission on Radiation Units and Measurements. ICRU Report 62: Prescribing, recording, and reporting photon beam therapy (Supplement to ICRU Report 50). Bethesda, MD: ICRU, 1999.

19. Stroom, J.C. and B.J.M. Heijmen, Geometrical uncertainties, radiotherapy planning margins, and the ICRU-62 report. Radiotherapy and Oncology, 2002. 64: p. $75-83$.

20. Hanley, J., M.M. Debois, D. Mah, G.S. Mageras, A. Raben, K. Rosenzweig, B. Mychalczak, L.H. Schwartz, P.J. Gloeggler, W. Lutz, C.C. Ling, S.A. Leibel, Z. Fuks, and G.J. Kutcher, Deep inspiration breath-hold technique for lung tumors: the potential value of target immobilization and reduced lung density in dose escalation. Int J Radiat Oncol Biol Phys, 1999. 45: p. 603-611.

21. Wong, J.W., M.B. Sharpe, D.A. Jaffray, V.R. Kini, J.M. Robertson, J.S. Stromberg, and A.A. Martinez, The use of active breathing control $(A B C)$ to reduce margin for breathing motion. Int J Radiat Oncol Biol Phys, 1999. 44: p. 911-919.

22. Ramsay, C.R., D. Scaperoth, D. Arwood, and A.L. Oliver, Clinical efficacy of respiratory gated conformal radiation therapy. Medical Dosimetry, 1999. 24: p. 115-119.

23. Vedam, S.S., P.J. Keall, V.R. Kini, and R. Mohan, Determining parameters for respiration-gated radiotherapy. Med Phys, 2001. 28: p. 2139-2146.

24. Suhy, J. and P.J. Maniawski. Routine clinical application of 4D Time-of-Flight PET/CT. 2011 [cited 2015 July 10]; Available from: clinical.netforum.healthcare.philips.com/us_en/Explore/WhitePapers/PetCT/Routine-clinical-application-of-4D-Time-of-Flight-PET-CT.

25. Murphy, M.J., Tracking moving organs in real time. Seminars in Radiation Oncology, 2004. 14: p. 91-100.

26. Ozhasoglu, C., C.B. Saw, H. Chen, S. Burton, K. Komanduri, N.J. Yue, S.M. Huq, and D.E. Heron, Synchrony - CyberKnife Respiratory Compensation Technology. Medical Dosimetry, 2008. 33: p. 117-123.

27. Soubra, M., J. Cygler, and G. Mackay, Evaluation of a dual bias dual metal oxide-silicon semiconductor field effect transistor detector as radiation dosimeter. Med Phys, 1994. 21: p. 567-572. 
28. Dieterich, S., C. Cavedon, C.F. Chuang, A.B. Cohen, J.A. Garrett, C.L. Lee, J.R. Lowenstein, M.F. d'Souza, D.D. Taylor, X. Wu, and C. Yu, Report of AAPM TG 135: Quality assurance for robotic radiosurgery. Med Phys, 2011. 38: p. 29142936.

29. Antypas, C. and E. Pantelis, Performance evaluation of a CyberKnife G4 imageguided robotic stereotactic radiosurgery system. Phys Med Biol, 2008. 53: p. 4697-4718.

30. Kilby, W., J.R. Dooley, G. Kuduvalli, S. Sayeh, and C.R. Maurer, The CyberKnife Robotic Radiosurgery System in 2010. Technol Cancer Res T, 2010. 9: p. 433-453.

31. Descovich, M., P.K. Sneed, N.M. Barbaro, M.W. McDermott, C.F. Chuang, I.J. Barani, J.L. Nakamura, and L. Ma, A dosimetric comparison between Gamma Knife and CyberKnife treatment plans for trigeminal neuralgia. J Neurosurg, 2010. 113: p. 199-206.

32. Sayeh, S., J. Wang, W.T. Main, W. Kilby, and C.R. Maurer, Respiratory Motion Tracking for Robotic Radiosurgery, in Treating Tumors that Move with Respiration, H.C. Urschel, Editor. 2007, Springer: Germany. p. 15-29.

33. Cherpak, A., W. Ding, A. Hallil, and J.E. Cygler, Evaluation of a novel $4 D$ in vivo dosimetry system. Med Phys, 2009. 36: p. 1672-1679.

34. Cherpak, A., Dose and Position Measurements using a Novel 4D In Vivo Dosimetry System, in Department of Physics. 2011, Carleton University: Ottawa, Ontario, Canada.

35. Hummel, J.B., M.R. Bax, M.L. Figl, Y. Kang, C. Maurer, W.W. Birkfellner, H. Bergmann, and R. Shahidi, Design and application of an assessment protocol for electromagnetic tracking systems. Med Phys, 2005. 32: p. 2371-2379.

36. Borca, V.C., M. Pasquino, G. Russo, P. Grosso, D. Cante, P. Sciacero, G. Girelli, M.R. La Porta, and S. Tofani, Dosimetric characterization and use of GAFCHROMIC EBT3 film for IMRT dose verification. J Appl Clin Med Phys, 2013. 14: p. 158-171.

37. van Battum, L.J., D. Hoffmans, H. Piersma, and S. Heukelom, Accurate dosimetry with GafChromic EBT film of a $6 \mathrm{MV}$ photon beam in water: What level is achievable? Med Phys, 2008. 35: p. 704-716.

38. Cheung, T., M.J. Butson, and P.K.N. Yu, Post-irradiation colouration of Gafchromic EBT radiochromic film. Phys Med Biol, 2005. 50: p. N281-N285.

39. Lewis, D., A. Micke, X. Yu, and M.F. Chan, An efficient protocol for radiochromic film dosimetry combining calibration and measurement in a single scan. Med Phys, 2012. 39: p. 6339-6350.

40. Low, D.A., W.B. Harms, S. Mutic, and J.A. Purdy, A technique for the quantitative evaluation of dose distributions. Med Phys, 1998. 25: p. 656-661.

41. Devic, S., J. Seuntjens, E. Sham, E.B. Podgorsak, C.R. Schmidtlein, A.S. Kirov, and C.G. Soares, Precise radiochromic film dosimetry using a flat-bed document scanner. Med Phys, 2005. 32: p. 2245-2253.

42. Arun, K.S., T.S. Huang, and S.D. Blostein, Least-Squares Fitting of Two 3-D Point Sets. IEEE T Pattern Anal, 1987. 9: p. 698-700.

43. Umeyama, S., Least-Squares Estimation of Transformation Parameters Between Two Point Patterns. IEEE T Pattern Anal, 1991. 13: p. 376-380. 
44. Physics Essentials Guide. 2010, Accuray Inc.

45. Sharma, S.C., J.T. Ott, J.B. Williams, and D. Dickow, Clinical implications of adopting Monte Carlo treatment planning for CyberKnife. J Appl Clin Med Phys, 2010. 11: p. 170-175.

46. Ma, C., J.S. Li, J. Deng, and J. Fan, Implementation of Monte Carlo Dose Calculation for CyberKnife treatment planning. J Phys Conf Ser, 2008. 102: p. 110.

47. Li, J.S., T. Pawlicki, J. Deng, S.B. Jiang, E. Mok, and C.-M. Ma, Validation of a Monte Carlo dose calculation tool for radiotherapy treatment planning. Phys Med Biol, 2000. 45: p. 2969-2985.

48. Wilcox, E.E. and G.M. Daskalov, Accuracy of dose measurements and calculations within and beyond heterogeneous tissues for $6 \mathrm{MV}$ photon fields smaller than $4 \mathrm{~cm}$ produced by Cyberknife. Med Phys, 2008. 35: p. 2259-2266.

49. Accuray, Estimation of the Imaging Dose for the CyberKnife Robotic Radiosurgery System. 2010.

50. Olson, J.R., C.J. Liu, Y. Tian, and Q. Shen, Theoretical Wood Densitometry: II. Optimal X-ray Energy for Wood Density Measurement. Wood and Fiber Science, 1988. 20: p. 187-196.

51. Johns, H. and J. Cunningham, The Physics of Radiology. 4th Edition ed. 1983, Springfield, IL: Charles C Thomas.

52. Brown, T.A.D., K.R. Hogstrom, D. Alvarez, K.L. Matthews, K. Ham, and J. Dugas, Dose-response curve of EBT, EBT2, and EBT3 radiochromic films to synchrotron-produced monochromatic $x$-ray beams. Med Phys, 2012. 39: p. 7412-7417.

53. Villarreal-Barajas, J.E. and R.F.H. Khan, Energy response of EBT3 radiochromic films: implications for dosimetry in kilovoltage range. J Appl Clin Med Phys, 2014. 15(331-338).

54. Massillon-JL, G., S. Chiu-Tsao, I. Domingo-Muñoz, and M.F. Chan, Energy Dependence of the New Gafchromic EBT3 Film: Dose Response Curves for 50 $k V$, 6 and $15 M V X$-Ray Beams. International Journal of Medical Physics, Clinical Engineering and Radiation Oncology, 2012. 1: p. 60-65.

55. Sutherland, J.G.H. and D.W.O. Rogers, Monte Carlo calculated absorbed-dose energy dependence of EBT and EBT2 film. Med Phys, 2010. 37: p. 1110-1116.

56. Hermida-López, M., L. Lüdemann, A. Flühs, and L. Brualla, Technical Note: Influence of the phantom material on the absorbed-dose energy dependence of the EBT3 radiochromic film for photons in the energy range $3 \mathrm{keV}-18 \mathrm{MeV}$. Med Phys, 2014. 41: p. 1-6.

57. Bekerat, H., S. Devic, F. DeBlois, K. Singh, A. Sarfehnia, J. Seuntjens, S. Shih, $\mathrm{X}$. Yu, and D. Lewis, Improving the energy response of external beam therapy (EBT) GafChromicTM dosimetry films at low energies ( $\leq 100 \mathrm{keV})$. Med Phys, 2014. 41: p. 1-14.

58. Wang, B., X.G. Xu, and C. Kim, Monte Carlo Study of MOSFET Dosemeter Characteristics: Dose Dependence on Photon Energy, Direction and Dosemeter Composition. Radiation Protection Dosimetry, 2005. 113: p. 40-46. 
59. McEwen, M., L. DeWard, G. Ibbott, D. Followill, D.W.O. Rogers, S. Seltzer, and J. Seuntjens, Addendum to the AAPM's TG-51 protocol for clinical reference dosimetry of high-energy photon beams. Med Phys, 2014. 41: p. 041501-(1-20).

60. Kawachi, T., H. Saitoh, M. Inoue, T. Katayose, A. Myojoyama, and K. Hatano, Reference dosimetry condition and beam quality correction factor for CyberKnife beam. Med Phys, 2008. 35: p. 4591-4598.

61. Araki, F., Monte Carlo study of a Cyberknife stereotactic radiosurgery system. Med Phys, 2006. 33: p. 2955-2963.

62. Ramaseshan, R., K.S. Kohli, T.J. Zhang, T. Lam, B. Norlinger, A. Hallil, and M. Islam, Performance characteristics of a microMOSFET as an in vivo dosimeter in radiation therapy. Phys Med Biol, 2004. 49: p. 4031-4048.

63. Cherpak, A., R.C.N. Studinski, and J.E. Cygler, MOSFET detectors in quality assurance of tomotherapy treatments. Radiotherapy and Oncology, 2008. 86: p. 242-250.

64. Ramaseshan, R., S. Russell, and P. O'Brien, Clinical Dosimetry Using MOSFETs. Int J Radiat Oncol Biol Phys, 1997. 37: p. 959-964.

65. Verellen, D., S. Van Vaerenbergh, K. Tournel, K. Heuninckx, L. Joris, M. Duchateau, N. Linthout, T. Gevaert, T. Reynders, I. Van de Vondel, L. Coppens, T. Depuydt, M. De Ridder, and G. Storme, An in-house developed resettable MOSFET dosimeter for radiotherapy. Phys Med Biol, 2010. 55: p. N97-N109.

66. Scalchi, P. and P. Francescon, Calibration of a MOSFET Detection System for 6MV In Vivo Dosimetry. Int J Radiat Oncol Biol Phys, 1998. 40: p. 987-993.

67. Tanyi, J.A., S.P. Krafft, T. Hagio, M. Fuss, and B.J. Salter, MOSFET sensitivity dependence on integrated dose from high-energy photon beams. Med Phys, 2008. 35: p. 39-47.

68. Wilcox, E.E. and G.M. Daskalov, Evaluation of GAFCHROMIC EBT film for CyberKnife dosimetry. Med Phys, 2007. 34: p. 1967-1974.

69. Seuntjens, J., M. Olivares, M. Evans, and E.B. Podgorsak, Absorbed dose to water reference dosimetry using solid phantoms in the context of absorbed-dose protocols. Med Phys, 2005. 32: p. 2945-2953.

70. International Standards Organization. Evaluation of measurement data - Guide to the expression of uncertainty in measurement. International Committee for Weights and Measures (CIPM): Paris, France.

71. Pepin, E.W., H. Wu, Y. Zhang, and B. Lord, Correlation and prediction uncertainties in the CyberKnife Synchrony respiratory tracking system. Med Phys, 2011. 38: p. 4036-4044.

72. Hoogeman, M., J. Prevost, J. Nuyttens, J. Poll, P. Levendag, and B. Heijman, Clinical Accuracy of the Respiratory Tumor Tracking System of the CyberKnife: Assessment by Analysis of Log Files. Int J Radiat Oncol Biol Phys, 2009. 74: p. 297-303. 